\title{
Flexible Residential Smart Grid Simulation Framework
}

by

\section{Wang Xiang}

A thesis submitted to the Faculty of Graduate and Postdoctoral Affairs in partial fulfillment of the requirements for the degree of

\section{Master of Applied Science in Electrical and Computer Engineering}

in

Ottawa-Carleton Institute for Electrical and Computer Engineering (OCIECE)

Department of Systems and Computer Engineering

Carleton University

Ottawa, Ontario, Canada

\author{
Supervised by \\ Thomas Kunz \\ Marc St-Hilaire
}

(C) Copyright 2013, Wang Xiang 
The undersigned recommend to the Faculty of Graduate and Postdoctoral Affairs Acceptance of the thesis

\title{
Flexible Residential Smart Grid Simulation Framework
}

\author{
submitted by \\ Wang Xiang \\ in partial fulfillment of the requirements for \\ the degree of Master of Applied Science in Electrical and \\ Computer Engineering
}

Chair, Howard Schwartz, Department of Systems and Computer Engineering

Thesis Co-supervisor, Thomas Kunz

Thesis Co-supervisor, Marc St-Hilaire 


\begin{abstract}
Different scheduling and coordination algorithms controlling household appliances' operations can potentially lead to energy consumption reduction and/or load balancing in conjunction with different electricity pricing methods used in smart grid programs. In order to easily implement different algorithms and evaluate their efficiency against other ideas, a flexible simulation framework is desirable in both research and business fields. However, such a platform is currently lacking or underdeveloped. In this thesis, we provide a simulation framework to focus on demand side residential energy consumption coordination in response to different pricing methods. This simulation framework, equipped with an appliance consumption library using realistic values, aims to closely represent the average usage of different types of appliances. The simulation results of traditional usage yield close matching values compared to surveyed real life consumption records. Several sample coordination algorithms, pricing schemes, and communication scenarios are also implemented to illustrate the use of the simulation framework.
\end{abstract}




\section{Acknowledgements}

I would like to express my great appreciation to Prof. Thomas Kunz for his great guidance and critical decisions that led to the choice of the research topic and finally the completion of my thesis. I am deeply impressed with his judgment skill, comprehensive knowledge, and logical thinking ability.

I would like to express my deep and sincere gratitude to Prof. Marc St-Hilaire for his great guidance, and for not giving me up after I obtained a full time work position and switched my master study to part time status. His critical judgments and continuous feedbacks led to my final completion of my thesis. I am also very impressed with his knowledge and judgment skill.

I am very proud to be supervised under my two professors. I am very glad with the choice of this interesting research topic, and enjoyed working on this thesis. Everything learnt in the course of this research project from them will be a role model in my profession career.

I owe my deepest thanks to my beloved parents who continuously provided me the ultimate supports and helped me through some difficult times both emotionally and financially. The unfair tuition fine almost put an end to my thesis study. It was them who helped me through those painful months. I will not be able to achieve my accomplishments today without them. 


\section{Table of Contents}

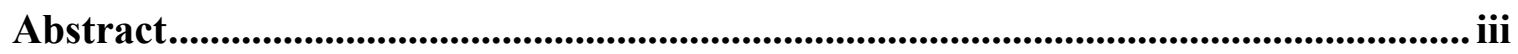

Acknowledgements ........................................................................................................................... iv

Table of Contents .................................................................................................................................. v

List of Tables ............................................................................................................................... viii

List of Figures.................................................................................................................................. ix

List of Acronyms ............................................................................................................................. xii

Chapter 1: Introduction ................................................................................................................. 1

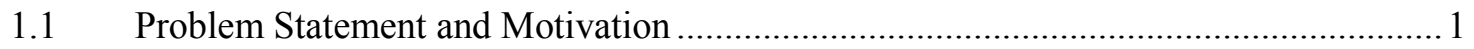

$1.2 \quad$ Research Objective .......................................................................................

$1.3 \quad$ Thesis Contributions......................................................................................

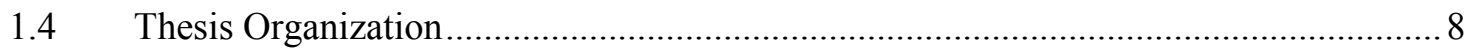

Chapter 2: Background and Related Work ........................................................................ 10

2.1 Smart Grid Definition and Components ................................................................. 10

2.2 Smart Appliance Coordination Technologies....................................................... 13

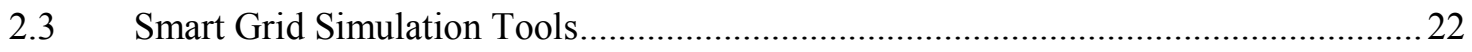

Chapter 3: Design and Implementation............................................................................. 27

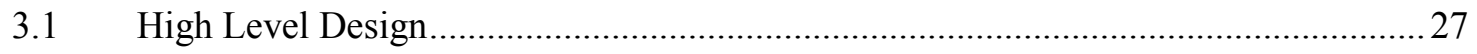

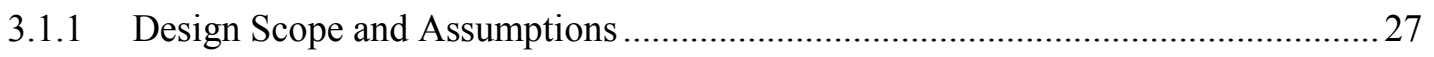

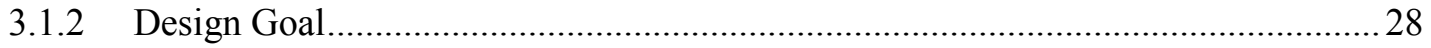

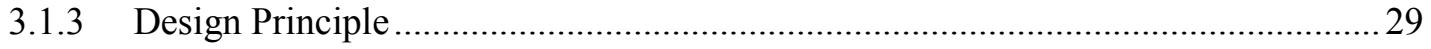

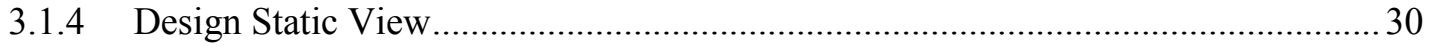

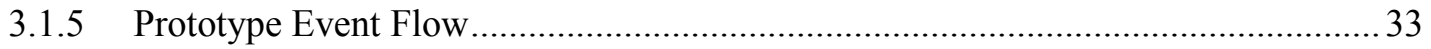

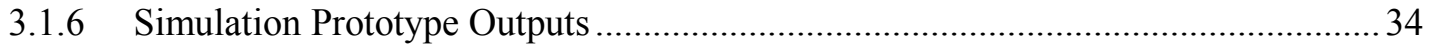




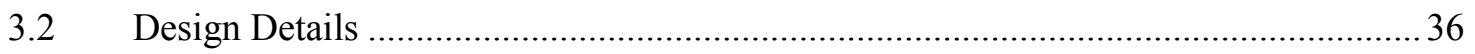

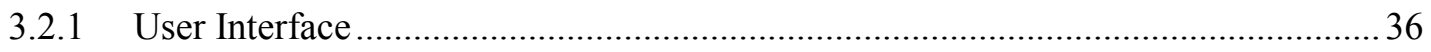

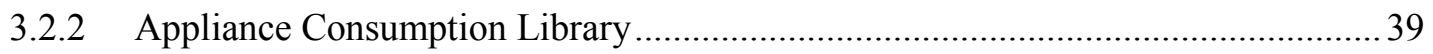

3.2.2.1 Time Constraint Attributes .......................................................................... 41

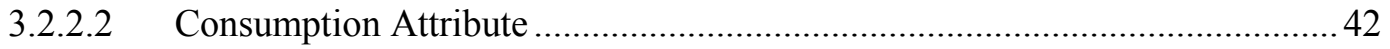

3.2.2.3 Duration, Remainder, and Use Frequency Attributes..................................... 43

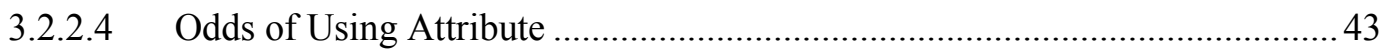

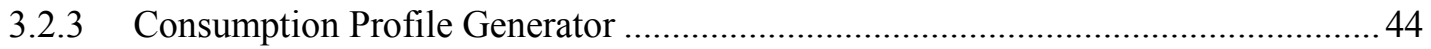

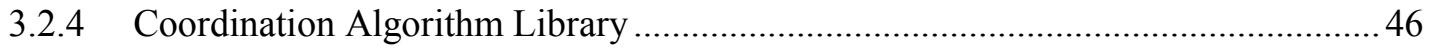

3.2.4.1 Traditional Appliances Consumption Generation .......................................... 47

3.2.4.2 Programmable Appliances Consumption Generation .................................... 47

3.2.4.3 Price Threshold Controlled Appliance Consumption Generation .................... 48

3.2.5 Time of Use (TOU) Price Provider .................................................................... 50

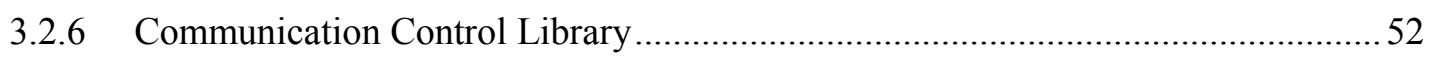

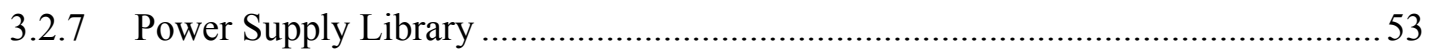

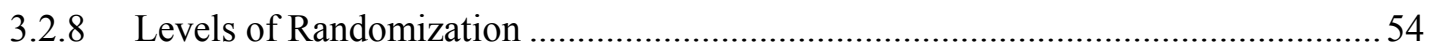

3.2.9 End of the Day Consumption Loopback …........................................................ 55

Chapter 4: Results and Validation ...................................................................57

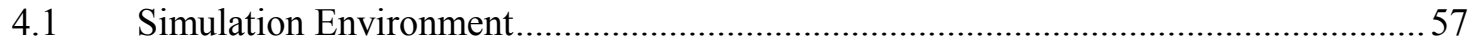

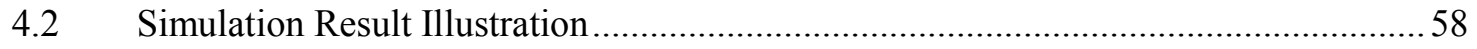

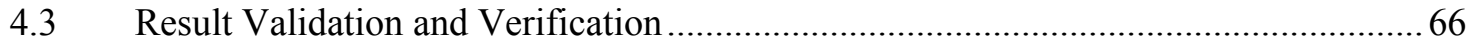

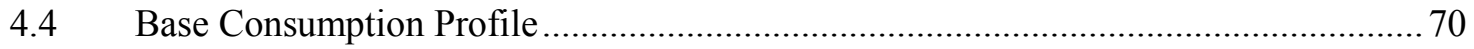

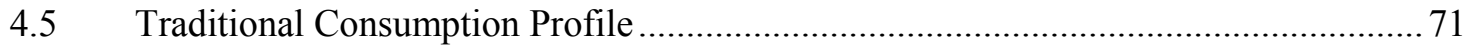

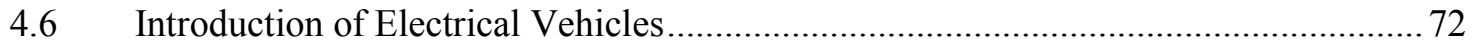

Chapter 5: Coordination and Pricing Schemes ......................................................... 75

$5.1 \quad$ Programmable Appliances Consumption Profile .................................................. 75 


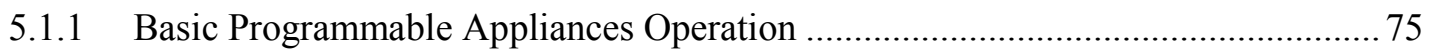

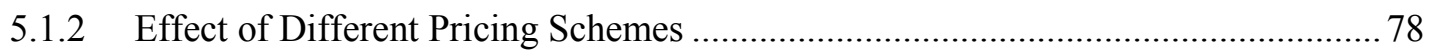

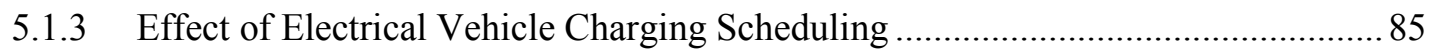

5.2 Threshold-Controlled Appliances Consumption Profile ..........................................91

5.2.1 Effect of Different Price Threshold Settings ....................................................... 95

5.2.2 Effect of Pricing Profile Manipulation ................................................................ 97

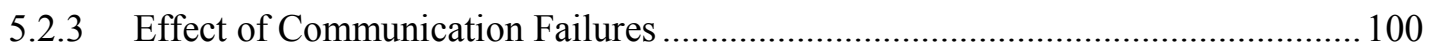

5.2.4 Effect of Different TOU Price Announcement Frequency ….............................. 102

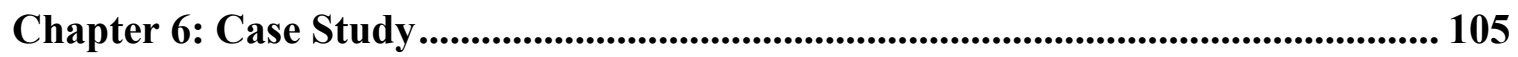

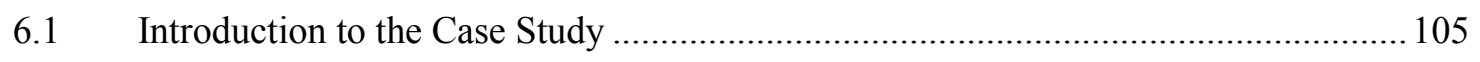

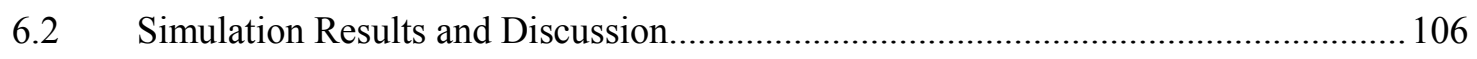

Chapter 7: Conclusion and Future Work........................................................................... 112

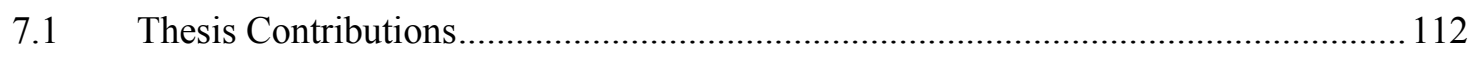

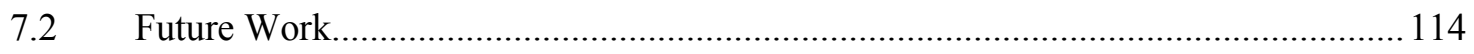

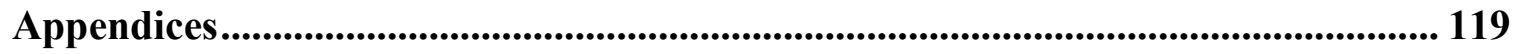

Appendix A - Appliance Consumption Table Implementation ............................................ 119

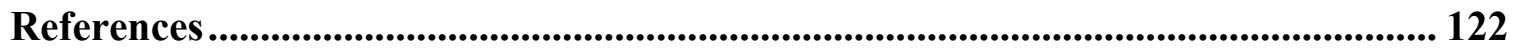




\section{List of Tables}

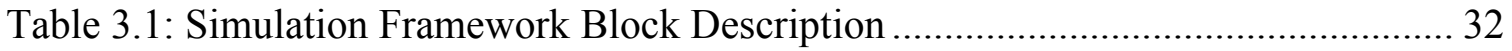

Table 3.2: Simulation Raw Data Table ........................................................................ 35

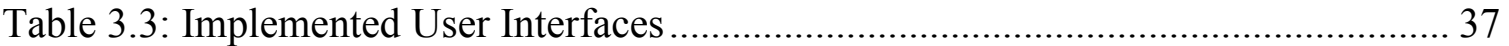

Table 3.4: Appliance Consumption Table Example ................................................. 41

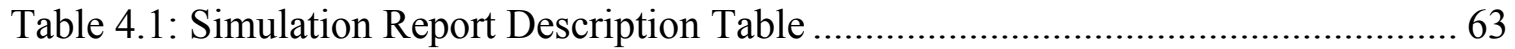

Table 6.1: Threshold Setting Iteration Table .......................................................... 107 


\section{List of Figures}

Figure 1.1: Consumer Questions about Smart Grid [5] ........................................... 2

Figure 1.2: Communication Testing Model with Multiple End Devices [15] .................. 4

Figure 1.3: CISCO Home Energy Controller [18] .................................................... 5

Figure 2.1: Smart Home Demonstration [23] ......................................................... 11

Figure 2.2: (a) Load and Reserves on a Typical U.S. Peak Day, (b) Residential Load

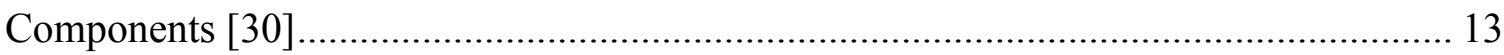

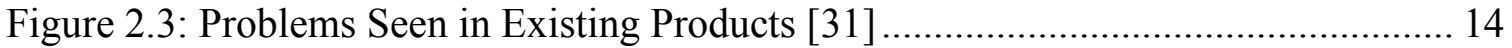

Figure 2.4: Framework of the Intelligent Agent Middleware for Context Aware System

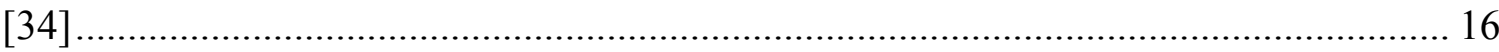

Figure 2.5: Energy Scheduler Model with Time-varying Price [35] ............................. 17

Figure 2.6: System Model for Within-houshold Coordination [41] ............................. 19

Figure 2.7: Autonomous Distributed V2G in Ubiquitous Power Grid [47] .................. 21

Figure 2.8: Mosaik Framework Layer Structure [49] ............................................... 23

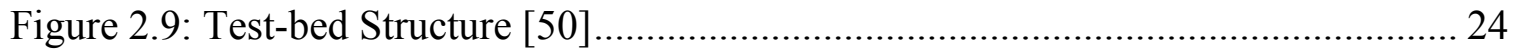

Figure 2.10: Simulation Flow Chart of [51] .............................................................. 25

Figure 3.1: Simulation Framework Block Diagram ................................................. 31

Figure 3.2: Base Consumption Generation Pseudo Logic ......................................... 45

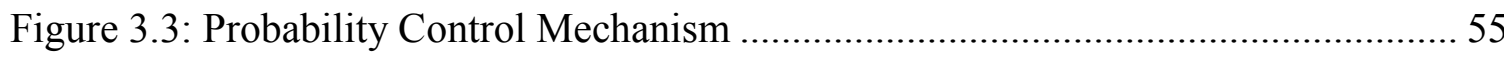

Figure 3.4: Example of Consumption Loopback ......................................................... 55

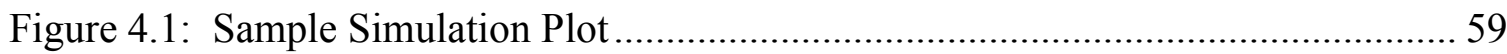

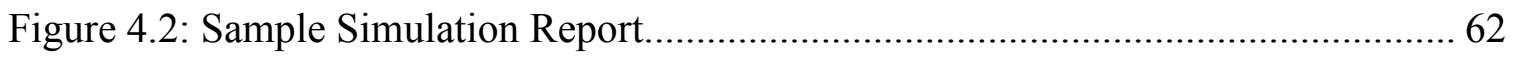


Figure 4.3: Average Daily Annual Electricity Consumption for Different House Types 68

Figure 4.4: Base Consumption vs. Traditional Consumption..................................... 71

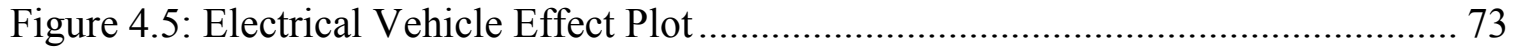

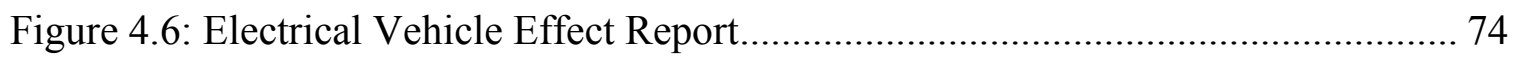

Figure 5.1: Programmable Appliance Consumption Profile........................................ 76

Figure 5.2: Programmable Appliance Consumption Report........................................ 76

Figure 5.3: Programmable Appliance Consumption Profile with OnPeak-MidPeak-

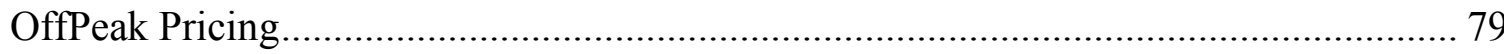

Figure 5.4: Programmable Appliance Consumption Report with OnPeak-MidPeak-

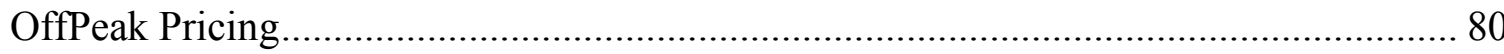

Figure 5.5: Programmable Appliance Consumption Profile with Linear Scale Pricing ... 82 Figure 5.6: Programmable Appliance Consumption Report with Linear Scale Pricing ... 83 Figure 5.7: EV Charging Scheduling Under OnPeak-MidPeak-OffPeak Pricing Sceme 86 Figure 5.8: EV Charging Scheduling Under OnPeak-MidPeak-OffPeak Report............ 87

Figure 5.9: Charging Scheduling Under Linear Pricing Sceme ................................. 88

Figure 5.10: Charging Scheduling Under Linear Pricing Scheme ............................... 89

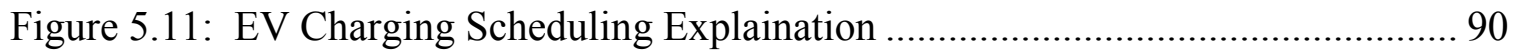

Figure 5.12: Threshold Controlled Appliance Consumption Plots............................... 92

Figure 5.13: Threshold Controlled Appliances Consumption Report ........................... 93

Figure 5.14: Threshold of 8 cent/kWh Consumption Plot ......................................... 96

Figure 5.15: Threshold of 8 cent/kWh Consumption Report ................................... 97

Figure 5.16: Price Profile Scaling Effect Plot......................................................... 98 
Figure 5.17: Price Profile Scaling Effect Report ...................................................... 99

Figure 5.18: Communication Failure Plot ........................................................... 100

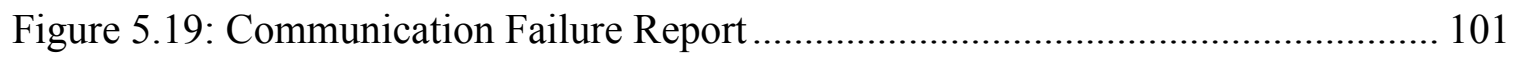

Figure 5.20: Effect of Decreasing the TOU Price Distribution Frequency Plot ............. 102

Figure 5.21: Effect of Decreasing the TOU Price Distribution Frequency Report ........ 103

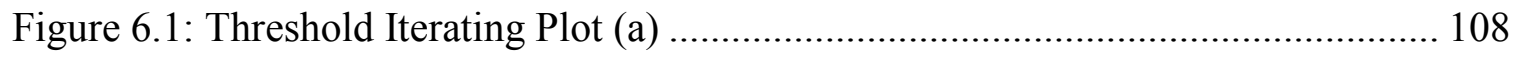

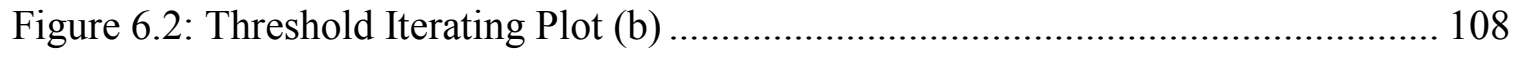

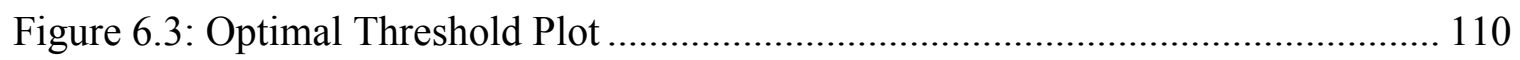




\section{List of Acronyms}

$\begin{array}{ll}\text { AMI } & \text { Advanced Metering Infrastructure } \\ \text { CI } & \text { Confidence Interval } \\ \text { DB } & \text { Database } \\ \text { DR } & \text { Demand Response } \\ \text { EV } & \text { Electrical Vehicle } \\ \text { FM } & \text { Frequency Modulation } \\ \text { EPRI } & \text { Electric Power Research Institute } \\ \text { GFA } & \text { Grid Friendly Appliance } \\ \text { HAN } & \text { Home Area Network } \\ \text { HVAC } & \text { Heating, Ventilating, and Air Conditioning } \\ \text { IEC } & \text { International Electrotechnical Commission } \\ \text { IEEE } & \text { Institute of Electrical and Electronics Engineers } \\ \text { LAN } & \text { Local Area Network } \\ \text { LFC } & \text { Load Frequency Control } \\ \text { NS-2 } & \text { Network Simulator Version-2 } \\ \text { OLE } & \text { Object Linking and Embedding } \\ \text { OPC } & \text { OLE for Process Control } \\ \text { PEV/PHEV } & \text { Time of Use Electric Vehicle/Plug-in Hybrid Electric Vehicle } \\ \text { SOC } & \text { TCP }\end{array}$


UDP

V2G

WLAN
User Datagram Protocol

Vehicle-to-Grid

Wireless Local Area Network 


\section{Chapter 1: Introduction}

In this chapter, we first introduce the topic of smart grid and describe the problem statement related to the availability of simulation environment. Then, the main research objectives are outlined followed by the research contributions. Finally, a general overview of the thesis is presented.

\subsection{Problem Statement and Motivation}

An emerging revolutionary change is happening in the power distribution industries around the globe, known as the smart grid development. Smart grid binds information network technologies into the outdated traditional power distribution network to enhance its resiliency and to reduce its carbon footprint. While smart grid is gradually becoming less of an unheard-of-phrase to more and more people due to governmental project roll out and various commercials and promotional activities all over the world [1] [2] [3] [4], the core concept of smart grid and its benefits are much less clear. This is especially true for general public customers. Several different groups and organizations conducted a few surveys around consumers' opinions [5] [6] [7] [8] [9]. The U.S. based Smart Grid Consumer Collaborative looked at over 80 research studies and white papers, and generated the "2011 State of the Consumer Report" [5]. It is stated in the report that only $28 \%$ of sampled individuals have a general understanding of what the smart grid really is, and what benefits it brings to both the environment and the economy. Off these $28 \%$, only $9 \%$ of them have enrolled in an electricity management program. Less than half of the consumers are aware of smart meters, even when those are deployed in their area. In the U.S., more than a quarter of the population does not know that they have the choice of 
purchasing energy from someone other than their local electric utility. Under this circumstance, the surveyed consumers were then asked about what kind of information they would find most valuable. The gathered responses, shown in Figure 1.1, reveal that consumers concern mostly about the cost of the technology and the savings that smart grid will produce, not surprisingly.

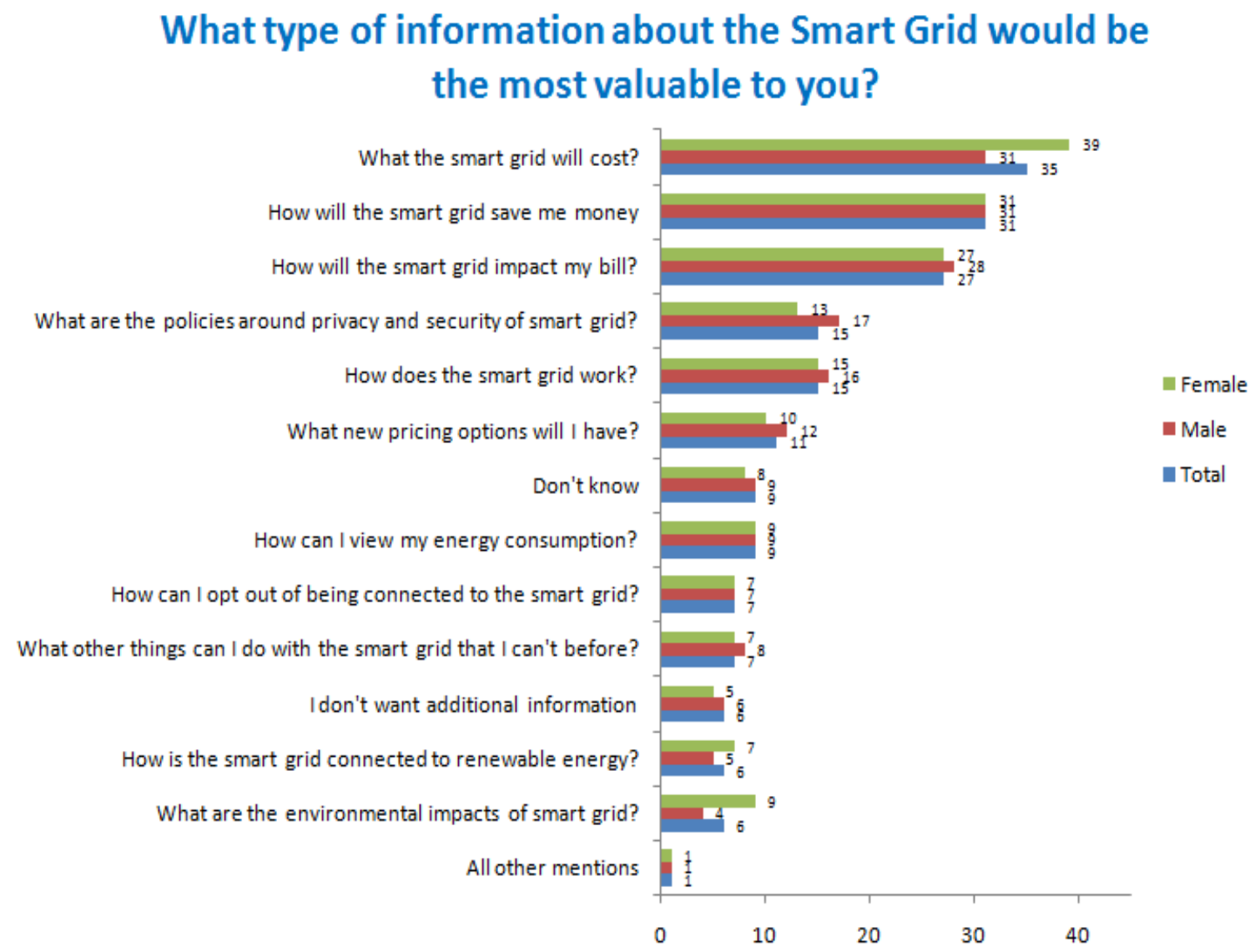

Figure 1.1: Consumer Questions about Smart Grid [5]

These harsh consumer requirements and expectations bring major challenges to smart grid advancement, but also lead to equally large possibilities and opportunities for research and business. The most relevant innovations to public consumers are the smart home and smart appliance technologies. The anticipated smart home is capable of 
automatically making smart decisions on energy consumption activities, and managing smart appliances to efficiently reduce energy consumption. Zpryme's report estimates that the market for smart appliances will grow from $\$ 3.06$ billion to $\$ 15$ billion by the year 2015 [10].

To capture a share of this pie, many enterprise solutions and new innovative solutions are being developed or have already been marketed over the past few years. For example, a foundation building block developer such as TalkingPlug has developed a power outlet that identifies the connected appliances, measures the power consumption, and sends the data to a server. A customer can then access this data through the Internet or their smart phones [11]. E-Radio inc. and CBC teamed up to develop a Time Of Use (TOU) price distribution solution via FM broadcast [12] [13]. Their solution utilizes the thorough radio station coverage in Ontario to achieve impressively comprehensive signal distribution with low cost. Groups such as the ClimateTalk Alliance, an organization of companies which develops a common communication infrastructure for interoperability among diverse systems [14] [15], work with the Electric Power Research Institute (EPRI) to develop a modular communication interface that enables any end device to work with any communication system to establish communication among each other with the module embedded. Figure 1.2 below illustrates their successfully verified test scenario with different appliances from different companies and brands. 


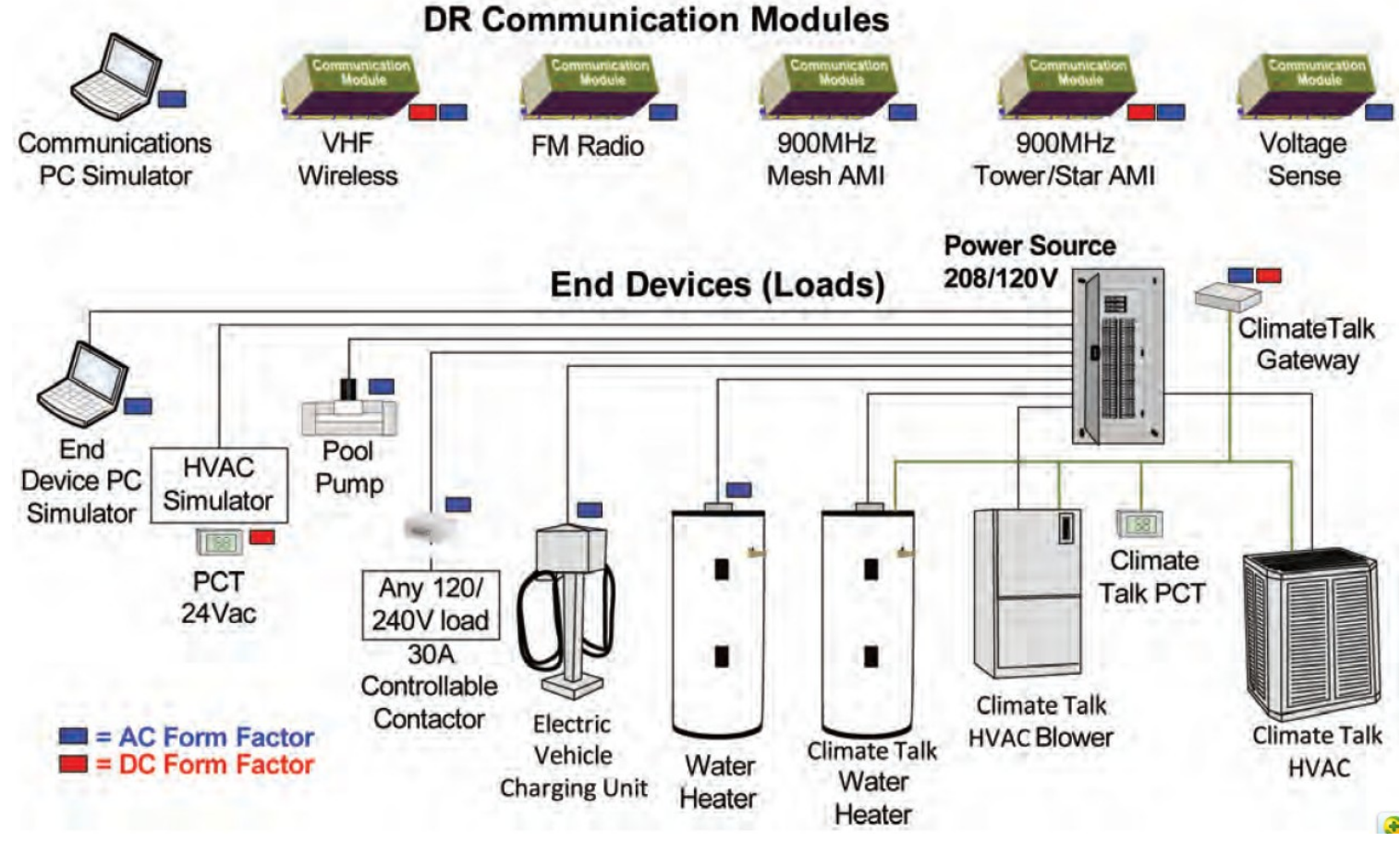

Figure 1.2: Communication Testing Model with Multiple End Devices [15]

Several companies are developing home energy management systems. For example, EcoFactor automates the control of home thermostats taking into consideration the temperatures at different regions of the home, the weather condition, and other information [16]. By continuously calculating and adjusting the room temperature, the technology is able to achieve aggregated saving of energy from each individual adjustment. Similarly, Cisco developed the first smart grid operating system, and marketed the Cisco Home Energy Controller [17], as shown in Figure 1.3 below. This operating system features a small countertop dashboard acting as a portal to household appliances' consumption information, controls, pricing information, and others. 


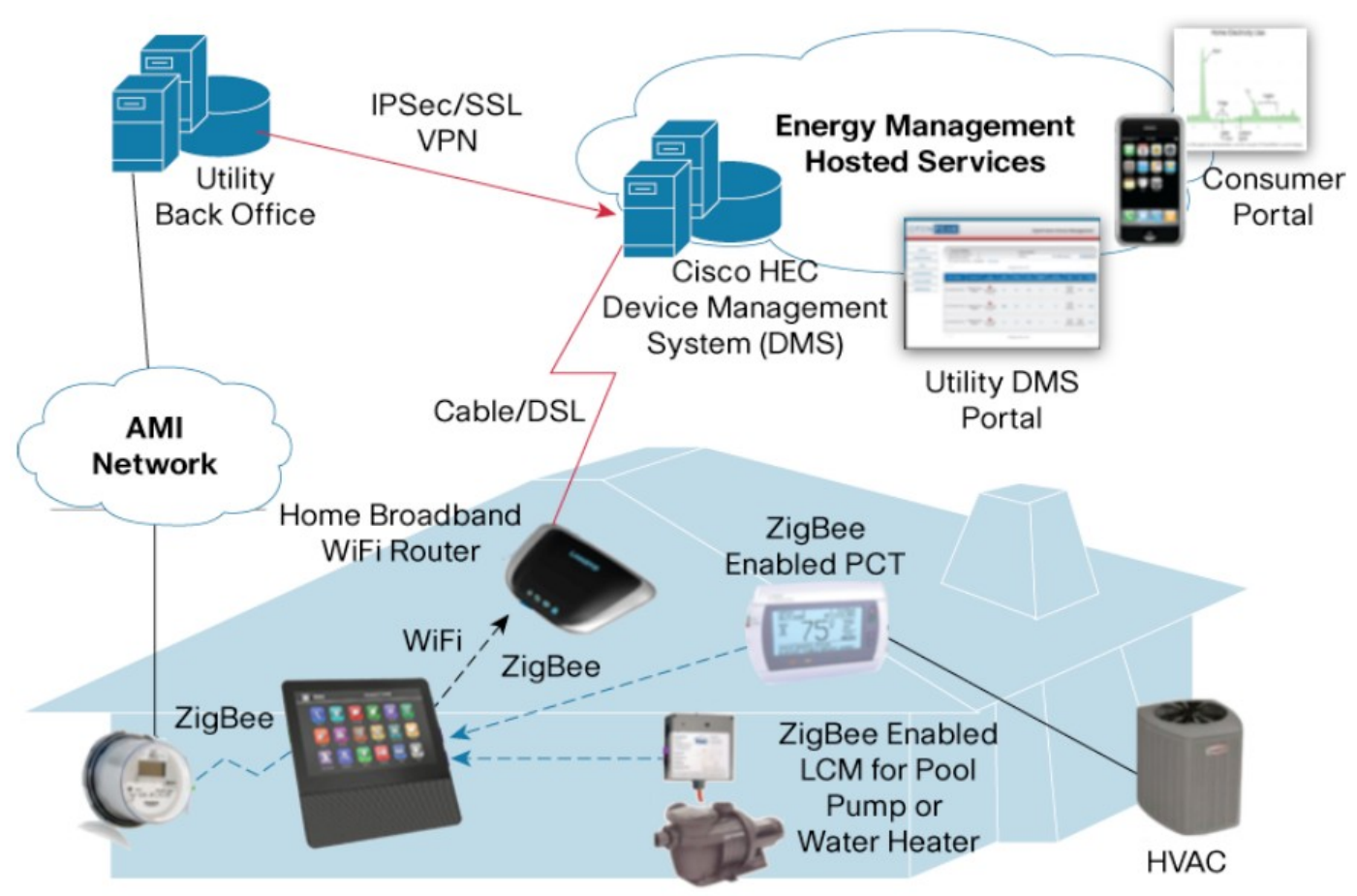

Figure 1.3: CISCO Home Energy Controller [18]

All the above innovative technologies aim at recreating a new household appliances operating system to comply with the smart grid. This new system is designed to enable information integration and operation management of the appliances. The enabled appliances can notify other appliances or control centers about their operation status; they can request information such as the electricity price; they can also alert users of any problem of their own or on the power grid. On the operation management side, these appliances can also be remotely controlled to start/stop their operation; or adjust their operation. More sophisticated systems have the appliances communicating and negotiating with others to collaboratively target different objectives such as to restrict energy consumptions or reduce the energy consumption costs. Having this new system in place, energy can be used where it is truly needed and at the time it is truly needed. 
However, all these proposals for energy saving, load balancing, and automatic fault recovery are not certain to achieve their objectives and to be widely adopted. The community currently lacks solid proofs/evidence on the desired benefits to answer consumers' questions. Information gathering and results evaluation in reality after the project/product deployment can be costly and most of time is not the best approach. Failure examples already occurred even with technology giants such as Microsoft and Google. Both companies developed their own web based energy consumption monitoring programs [19] [20] without understanding the real need from consumers. Both companies dropped their developed products by the middle of 2011 [21].

Therefore, it is desirable to have a smart grid centric test/simulation platform that is multipurpose and easy to expand to meet different needs. Whether developers need to evaluate the results of their product, or utility companies want to examine the effects of changing price profiles, it is beneficial to have a simulation framework that already implements the general energy consumption model, supply model, and information communication model. For example, government agencies can use this simulation framework as the basis to compare different project proposals or regulatory rules, and consumers can use it to aid their decision making on whether to change their consumption habits. After conducting a literature survey on this topic, we only found a limited number of related works. However, existing solutions either do not meet the desired functionality or they are proprietary solutions. Therefore, we propose a simulation framework to provide a flexible, extensible, and non-proprietary solution. 


\subsection{Research Objective}

The main objective of this thesis is to propose and develop a flexible simulation framework to study the behaviors and impacts of smart grid enabled household appliances. This proposed simulation framework should be accurate enough to reflect the general or average behaviors of traditional patterns of energy consumption. It should be designed to be flexible to allow expansions for other purposes, such as a study of the integration of renewable energy sources. It should be scalable to enable metro scale and also precise individual household simulations.

\subsection{Thesis Contributions}

The main contributions of this thesis are as follow:

- Household appliances consumption modeling - we put together a library containing the basic power consumption characteristics of common household appliances. The appliances are categorized into two groups: operation time nonshiftable and shiftable. Non-shiftable appliances are defined as those which have to start their operations immediately when needed. In contrast, shiftable appliances may postpone or reschedule their operation time. Such time-shiftable appliances are of particular interest to the smart grid technology.

- Demand side centric flexible simulation framework proposal - we propose a simulation framework that is open to integration of different components under the smart grid infrastructure, and puts more emphasis on the residential demand side of the story. This framework aims at providing an easy interface for 
implementing different appliance coordination algorithms and performing simulation tests with minimal effort.

- Simulation framework prototype implementation - we develop an initial version of the proposed simulation framework that is complete to demonstrate the usage of the framework and able to illustrate some findings from simple appliance coordination algorithms. This initial version of the simulation framework is ready to use and can be expanded to satisfy other needs.

- Case study demonstration - we provide a case study example to illustrate how to use the simulation framework iteratively to arrive at a desired goal. In our example we are interested in exploring policy alternatives/pricing strategies for the smart grid. An optimal threshold price is found to maximize the load stability without sacrificing customers' comfort.

\subsection{Thesis Organization}

The rest of this thesis is structured as follows: Chapter 2 provides some background information on the matter of study, and provides an overview of the state of art research and products that are relevant to the thesis. Then, Chapter 3 describes the design and implementation of the proposed simulation framework in detail. High level design goals and design decisions are discussed in this chapter. After that, Chapter 4 illustrates the simulation results obtained from the implemented simulation framework, and validates the results. Correctness and accuracy of the data are discussed in this chapter. Followed 
by that, Chapter 5 is dedicated to demonstrate the simulation results from a few simple appliance coordination methods. Analysis and discussions are performed to compare different implemented coordination methods. To demonstrate how to use the simulation framework, Chapter 6 provides a case study example to find an optimal price scheme to minimize the peak to average ratio with respect to energy consumption profiles. Lastly, Chapter 7 summarizes and concludes the thesis, and outlines future work on the topic. 


\section{Chapter 2: Background and Related Work}

To better understand the scope of the research, this chapter first clarifies the definition of smart grid, smart home, and smart appliances. Followed by that, a brief overview of works done on smart appliance coordination algorithms will be presented. Lastly, existing simulation tools and frameworks will be compared and discussed.

\subsection{Smart Grid Definition and Components}

The Canadian Electricity Association states that "The smart grid takes the existing electricity delivery system and makes it 'smart' by linking and applying seamless communications systems that can: gather and store data and convert the data to intelligence; communicate intelligence omnidirectionally among components in the 'smart' electricity system; and allow automated control that is responsive to that intelligence" [22]. With this intelligence, the smart grid aims to achieve better resilience, better operational efficiency, and a lower carbon foot print. Smart decisions will be made automatically upon detecting any problem in the grid, and recovery operation will also be automated. The need to construct new power plants will be reduced as better management and coordination of the energy consumption and generation activities will reduce peak load demands. The overall improved efficacy will reduce carbon emission, as more predictable energy consumption patterns will be matched with low-carbon energy generation capacities, rather than highly carbon-intensive energy generators such as coalfired power plants. 
Under this grid infrastructure, smart homes and smart appliances refer to those able to integrate and respond to the communication component of the smart grid to become part of the intelligence. They are able to optimize power usage based on electricity pricing information, weather condition, tenant occupancy, and other conditions to collectively achieve better efficiency. They automate the decision on appliance operations and the selection of energy source (power transmission line/local renewable energy/battery).

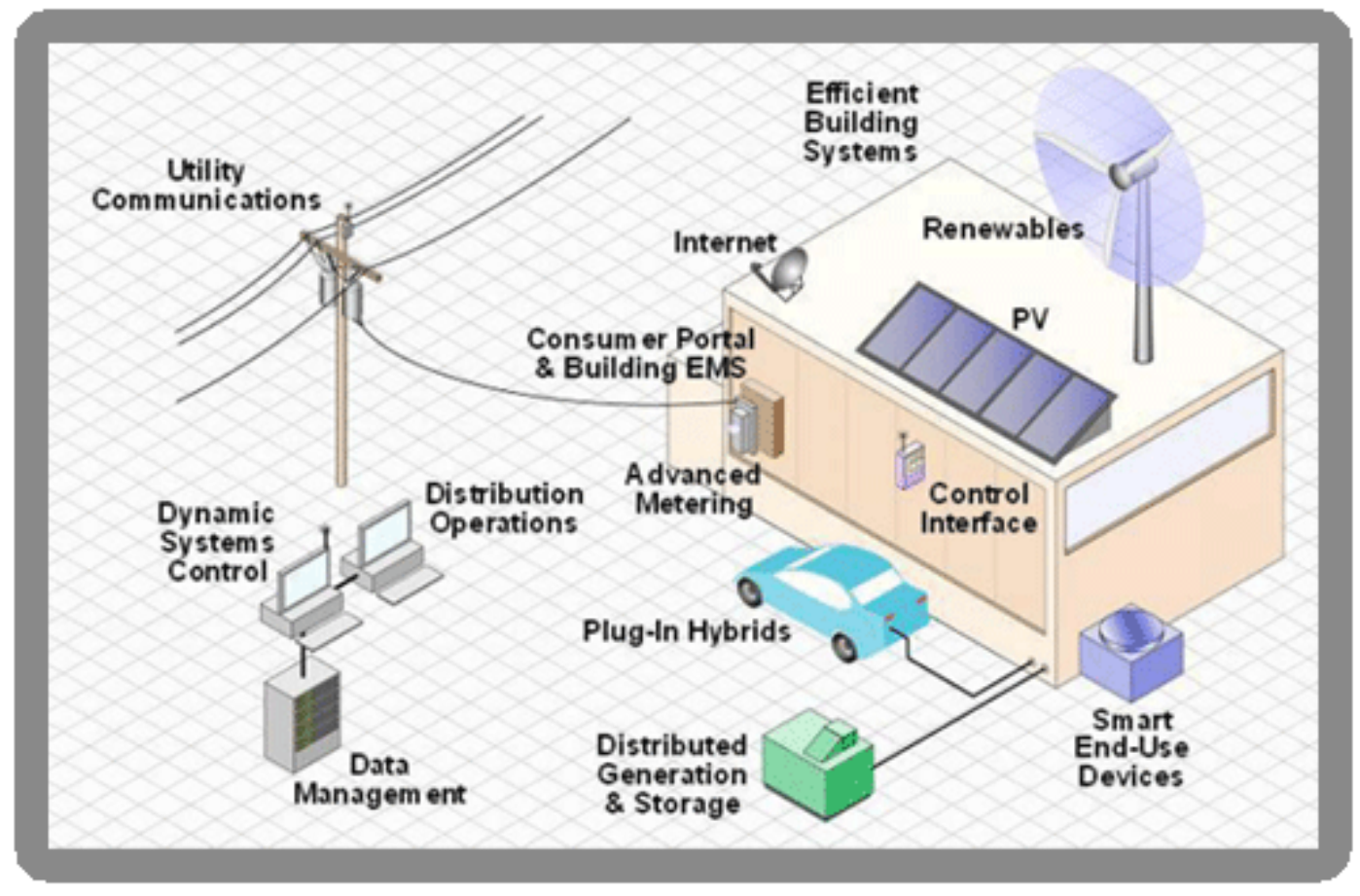

Figure 2.1: Smart Home Demonstration [23]

As shown in Figure 2.1, a primitive conceptual smart home is integrated into the Advanced Metering Infrastructure (AMI), or Internet/FM radio. The AMI is largely designed to enable communication of TOU pricing signals and consumption readings between the smart meters and control centers. Utilizing the AMI system, a smart home intelligently schedules the smart appliances' operating time. Under suitable conditions for local renewable energy sources, such as solar panels and wind mill, a smart home 
efficiently manages the usage and integration of those energies. Upon power outages, a smart home switches the energy source to renewable sources or batteries, such as an electric vehicle. In addition, if there is surplus energy generated locally, a smart home pushes energy back to the power grid to serve those who are in need [24].

Smart homes heavily rely on the development of a communication system for home area networks. AMI is not the only means of communication technology, and in fact, heterogeneous appliances and devices produced by different companies are using different communicating methods. Therefore, one ongoing field of research and development in the smart grid world is interoperability standards and protocols development. Governmental bodies such as the Canadian National Committee of the International Electrotechnical Commission (IEC) are working with several forum organizations to establish the principle standards. Their activities are led and regulated by the Standards Council of Canada [25]. Well-known organizations such as the IEEE are standardizing wireless home area networks [26]. Forum organizations such as the Zigbee Alliance and WiFi special interest groups are also working towards making their technologies become the future standard. Cloud computing is also considered as a serious contender in standardization. Several interoperability groups and organizations are tackling different levels of interoperability issues [27] [28] [29]. All these efforts, focusing on the basic connectivity level, aim at simply providing a common communication medium to allow data exchange between heterogeneous devices; whereas the goal for the network level aims at enabling data exchange between various networks. Nonetheless, all these developments are still in their development/standardization phase. 
There is not yet an obvious winner/de-facto established standard. Smart grid product developments and research studies hence need to take these into consideration to either study which technology they want to use, or make their work as independent as possible of the underlying communication technology.

\subsection{Smart Appliance Coordination Technologies}

One main objective of the smart grid is to achieve better energy efficiency. Energy should be generated at the time it is needed or at least used at the time the operation is truly needed. This management and coordination of energy generation and consumption is the interest of many research papers. In [30], the authors discuss the effectiveness of deploying Grid Friendly Appliances (GFA) in a competitive electricity market. They point out the potential benefits of controlling household appliance operations, as shown in the figure below.

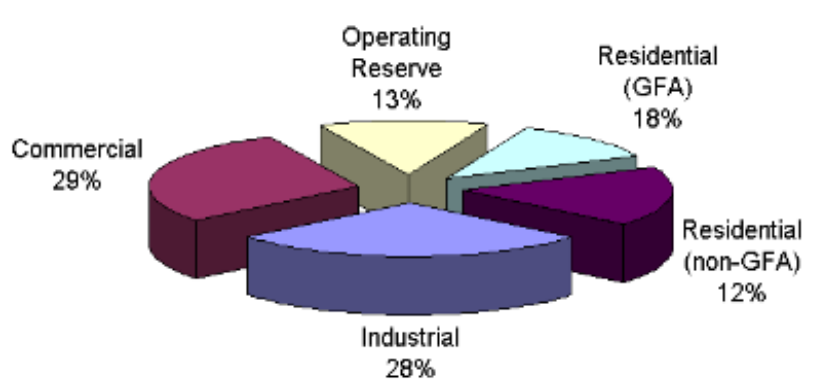

(a)

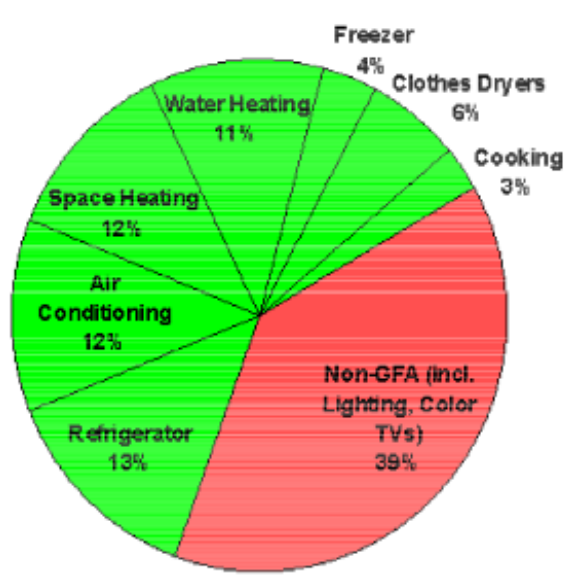

(b)

Figure 2.2: (a) Load and Reserves on a Typical U.S. Peak Day, (b) Residential Load Components [30] 
As shown, there is $18 \%$ power being consumed by the GFAs, which are subject to load control under the smart grid infrastructure. This is why many researchers are involved in GFA development and coordination method developments. In [31], the authors explore the benefits of integrating sensor network technology into the home area network to automatically adjust thermostat settings to reflect the occupancy status and thus to minimize the waste of energy. They heavily focused on studying the residential heating, ventilation and cooling system and point out several problems seen in existing systems, such as energy waste due to slow response and shallow setback. The figure below illustrates the problems caused by programmable thermostat operations and reactive thermostat operations.

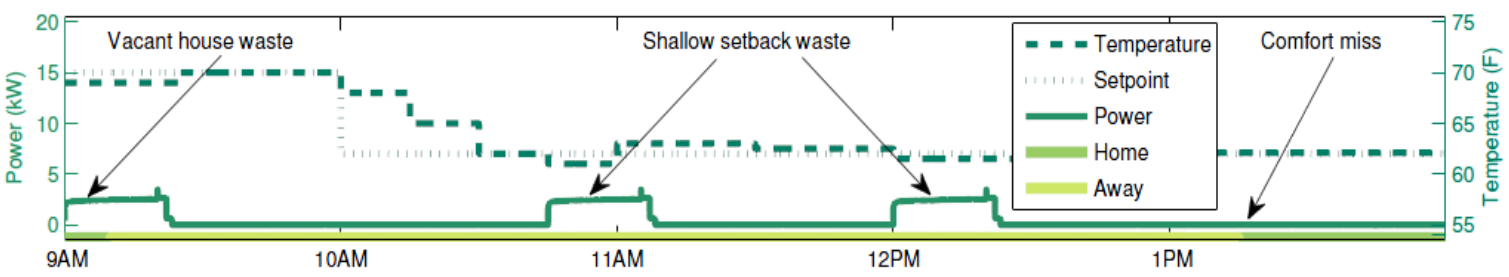

(a) Programmable Thermostat Operation

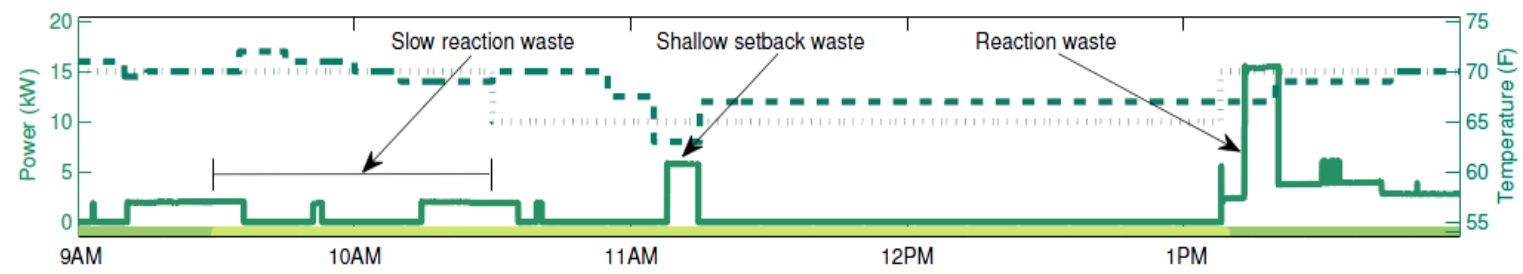

(b) Reactive Thermostat Operation

Figure 2.3: Problems Seen in Existing Products [31]

There are a few problems presented in Figure 2.3 that are seen in existing thermostat products. First, "Vacant house waste" is caused by maintaining the household temperature even after the occupants have already left the house. The "Shallow setback waste" is caused by a relatively high temperature setting while there is no occupant at 
home. "Comfort miss" occurs when the occupant returns home before the scheduled return home time. In the bottom plot, the "Slow reaction waste" is caused by poor reactive system that is not able to accurately determine the occupant status of the house. "Reaction waste" on the other hand occurs in the short period after the detection of the return of the occupant. In this short period the appliance (space heater in this case) needs to be operating at a high setting to bring the temperature back to the setpoint quickly.. To solve the problems shown in Figure 2.3, the authors propose an algorithm to detect and learn the behaviors of the occupants and then test their control algorithm to realize an energy saving of $28 \%$. Not only that, the authors also examine the level of comfort loss measured by how often the automated temperature does not meet the occupant requirements. In the end, their solution features a fast response time, large energy saving, and minimal comfort loss. Similar work has been done by many other researches. Authors in [32] employ intelligent agent theories in inhabitant action prediction algorithms. Their proposed smart home architecture is able to learn inhabitant's living patterns and automates the decision process using several prediction algorithms such as sequence matching, task-based Markov model, episode discovery, etc. They conducted small experiments to automate the actions of blinds to mainly optimize the comfort for inhabitants. In another paper [33], the authors propose a smart home area network architecture along with a management system to help consumers to use their energy more efficiently and minimize their personal impact on the environment. The above two papers are more or less based on a context aware intelligent agent middleware framework that is presented in [34]. In fact, the authors of [34] propose a framework that is able to adapt contexts in various types with software agent to provide appropriate services to users for 
different purposes. Learning and predicting from sensed data or other sources of input plays a key role in the proposed framework. Figure 2.4 illustrates the structure and relationship of the proposed framework. Starting from the bottom left of the diagram, the context integrator module converts information, obtained from the sensors and user inputs, into a normalized data structure defined by the authors. The normalized data structures are the current contexts and are also stored in the history DB. The reasoning engine then infers the current contexts into higher level contexts according to the predefined rules and knowledge. The higher level contexts are then used by the learning engine module to compute new rules and knowledge for prediction service. The context prediction module then uses suitable prediction algorithms to meet individual purposes. Lastly, the access control module grants data accessing and debugging with authentication policies.

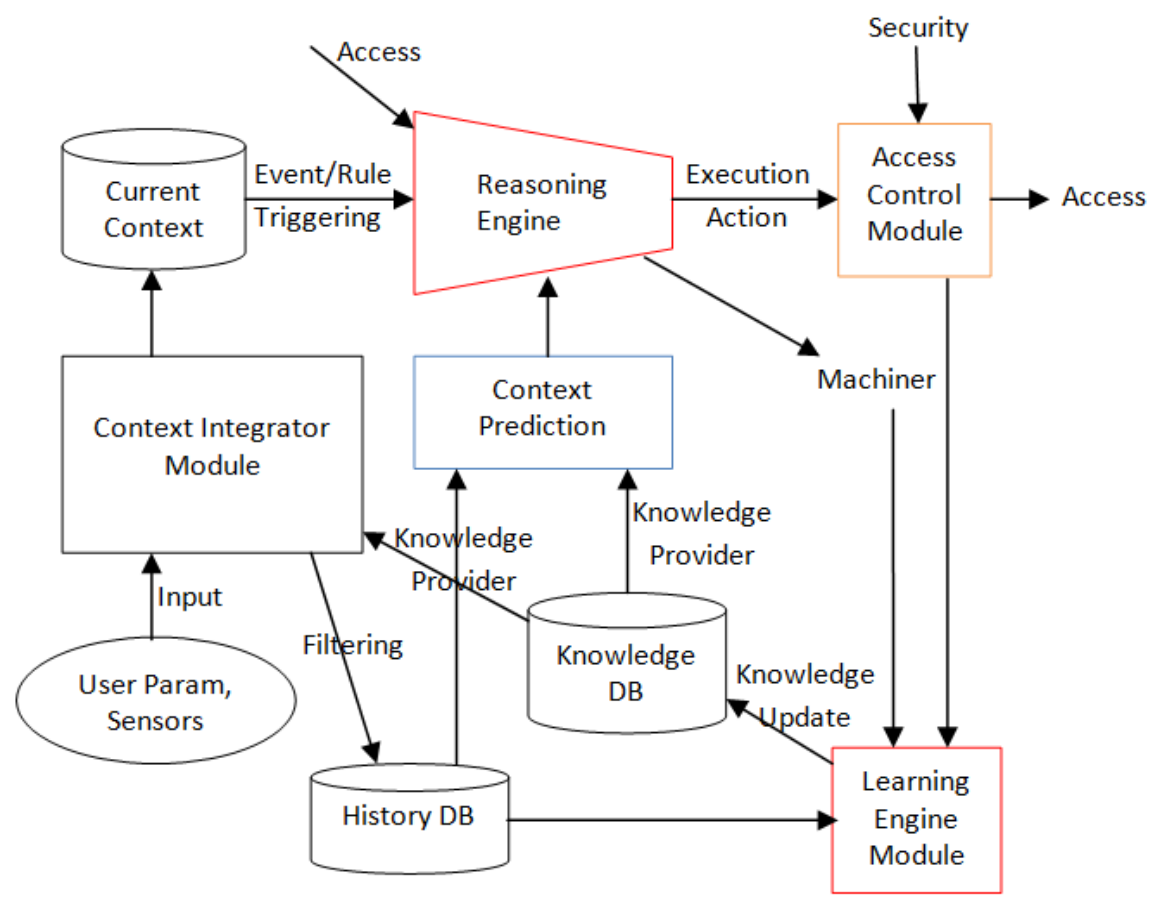

Figure 2.4: Framework of the Intelligent Agent Middleware for Context Aware System [34] 
However, all the above studies only focus on self-aware single household consumption management and/or optimization. They lack the knowledge from other components of the smart grid. How do their products respond to dynamic price schemes? What happens if many households in the neighborhood use the same coordination/management method? Will that cause other problems at larger scale or can their innovations further improve with the knowledge and coordination with other parties on the grid? How do their solutions integrate with the energy supply side such as renewable energies? Some of these questions are studied by other researches such as [35] which proposes an automatic energy consumption scheduling framework to balance the trade-offs between minimizing energy cost and minimizing the waiting time for appliance operations. The authors also realize the need for a price prediction capability in the presence of a time-varying pricing scheme and implemented a simple algorithm. Figure 2.5 illustrates their proposed solution. The TOU price from a utility is received from the LAN connection. The energy scheduler takes the TOU price and the predicted price to determine an optimal consumption schedule for the considered appliances.

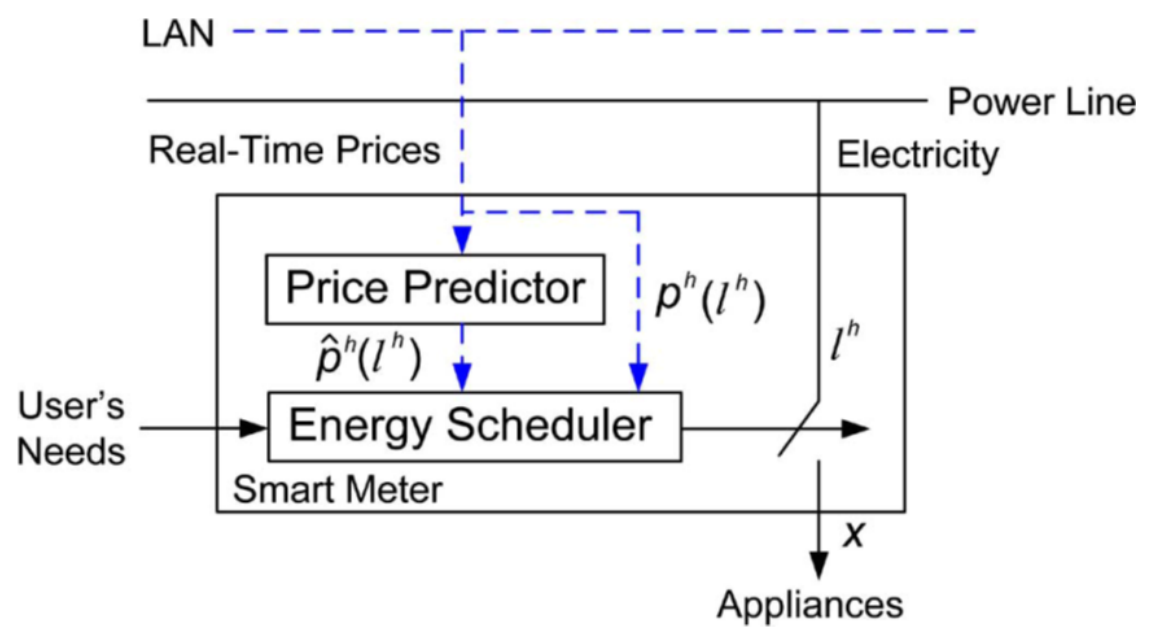

Figure 2.5: Energy Scheduler Model with Time-varying Price [35] 
A very similar work is conducted in [36] where the authors also propose a dynamic scheduling system to improve demand response in residential load control. In their paper, a prediction algorithm is also employed. However, instead of predicting the TOU price, [36] predicts the appliances' operation status with the help from a historical appliance operation database and fuzzy model. What is also included in the paper is a model of the solar power supply side to study its influence. Many more research projects are devoted to provide their solution to residential load control. [37] focuses on tackling the load uncertainty challenge on the residential consumption. [38] [39] and [40] model the energy management system as mathematical optimization problems and provided their solutions to the problems. Different equations and factors are considered in each study.

Some researchers take a step further and study the optimal appliance scheduling among neighborhoods. For example, [41] [42] [43] approach the problem by employing game theory and the prisoner's dilemma to achieve the Nash equilibrium among different sets of goals. The paper [41] describes two levels of coordination which are within-household appliances operation management and inter-household coordination. Figure 2.6 is an illustration of the first level of coordination where the goal is to achieve equilibrium between user preference and load conditions along with consumption characteristics of different appliances. As shown in Figure 2.6, the proposed system utilizes the smart meter as the central control component, and all other components of the system work around the smart meter. At the top, the user interface is majorly responsible for collecting user preference and displays the scheduled results. On the other side, the smart meter is connected to home appliances for gathering consumption information. With all the 
gathered information, the smart meter communicates to the central control to request an optimal schedule for appliances operations. Game theory is thus employed in the central control component. The one last component in Figure 2.6 illustrates the smart meter of other household(s) to achieve a collectively optimal scheduling among neighborhood. The simulation results presented in the paper demonstrate that the proposed system is able to achieve collectively better power/consumption balance among households.

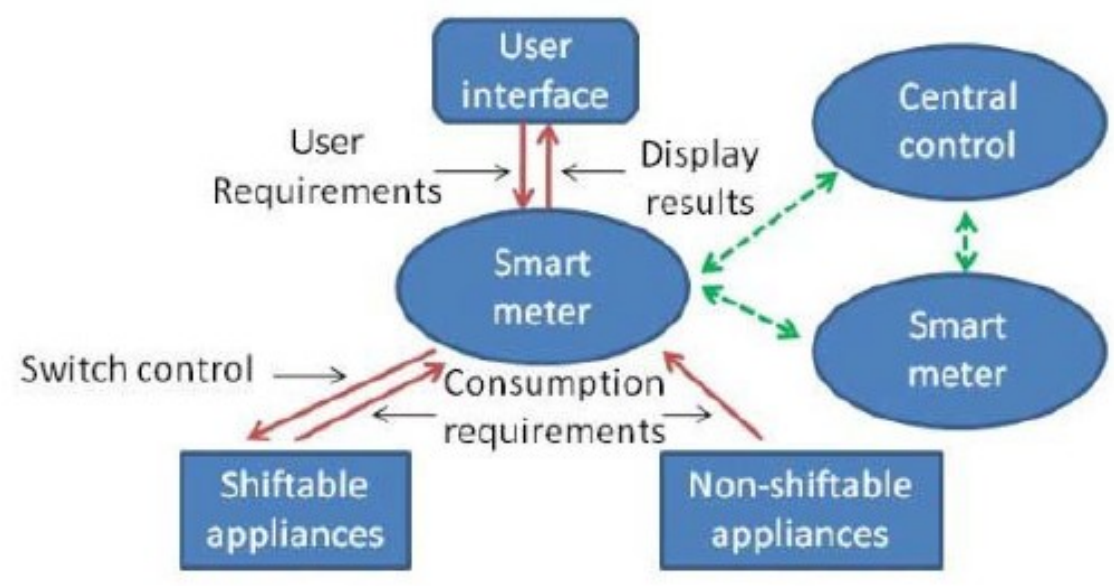

Figure 2.6: System Model for Within-houshold Coordination [41]

Instead of studying whole-house intelligent control, other researchers focus on a specific types of appliance operation across households such as charging of electric vehicles. The authors of [44] first developed an electrical vehicle charging model based on electrical properties of battery technologies and charging circuits, and then employed a conditional scheduling coordination method to push the charging operations overnight for the electrical vehicles that are not critically low in electricity storage. To achieve better control, the authors of [45] provide a theoretical model of a master-slave type of coordination mechanism between a control station and individual electrical vehicles. The control station listens to requests from electrical vehicles to start their charging, and 
approves or rejects the requests based on the provisioned amount of energy available. They further refine the work to reduce the rejection probability. A similar approach is seen in [46] where the authors define a three step procedure for the master controller to optimize the waiting time of the electrical vehicle fleet and the cost of the charging. The first step is called aggregation, in which individual electrical vehicle charging constraints are gathered and put into a tree structure. Next, in the optimization step, a charging plan is computed to minimize the costs for individual charging. Lastly, in the real-time control step, an incentive signal is sent to all electrical vehicles for their charging schedule.

Opposite to power drain, electrical vehicles can also be considered as a power source or a power buffer due to their large capacity batteries. The full impacts of the introduction of electrical vehicles are studied in [47]. In their research, they address the importance of vehicle-to-grid (V2G) to smart grid renewable energy sources. In the paper, an autonomous distributed $\mathrm{V} 2 \mathrm{G}$ control scheme is proposed to balance the power supply and demand on the grid. Figure 2.7 illustrates their system view of the grid. The Plug-in Hybrid Electric Vehicle (PHEV) and Electric Vehicle (EV) model is connected to the "Regional Load Dispatching Center" of the "Small Power Grid/Microgrid" portion. The $\mathrm{PHEV} / \mathrm{EV}$ serves three purposes in this system as demonstrated in the three ovals at bottom left of the figure. "Electric Vehicle Charging Request" obviously asks for power intake from the load dispatcher. "Battery Conditioning" is a service of keeping track of individual PHEV/EV's state-of-charge (SOC) and report to the dispatching center. Lastly, the "Contribution to LFC" in the power grid deals with pushing electricity back to the 
power grid for Load Frequency Control (LFC) purpose. Overall, the PHEV/EV is modeled as both a power taker and giver in the smart grid.

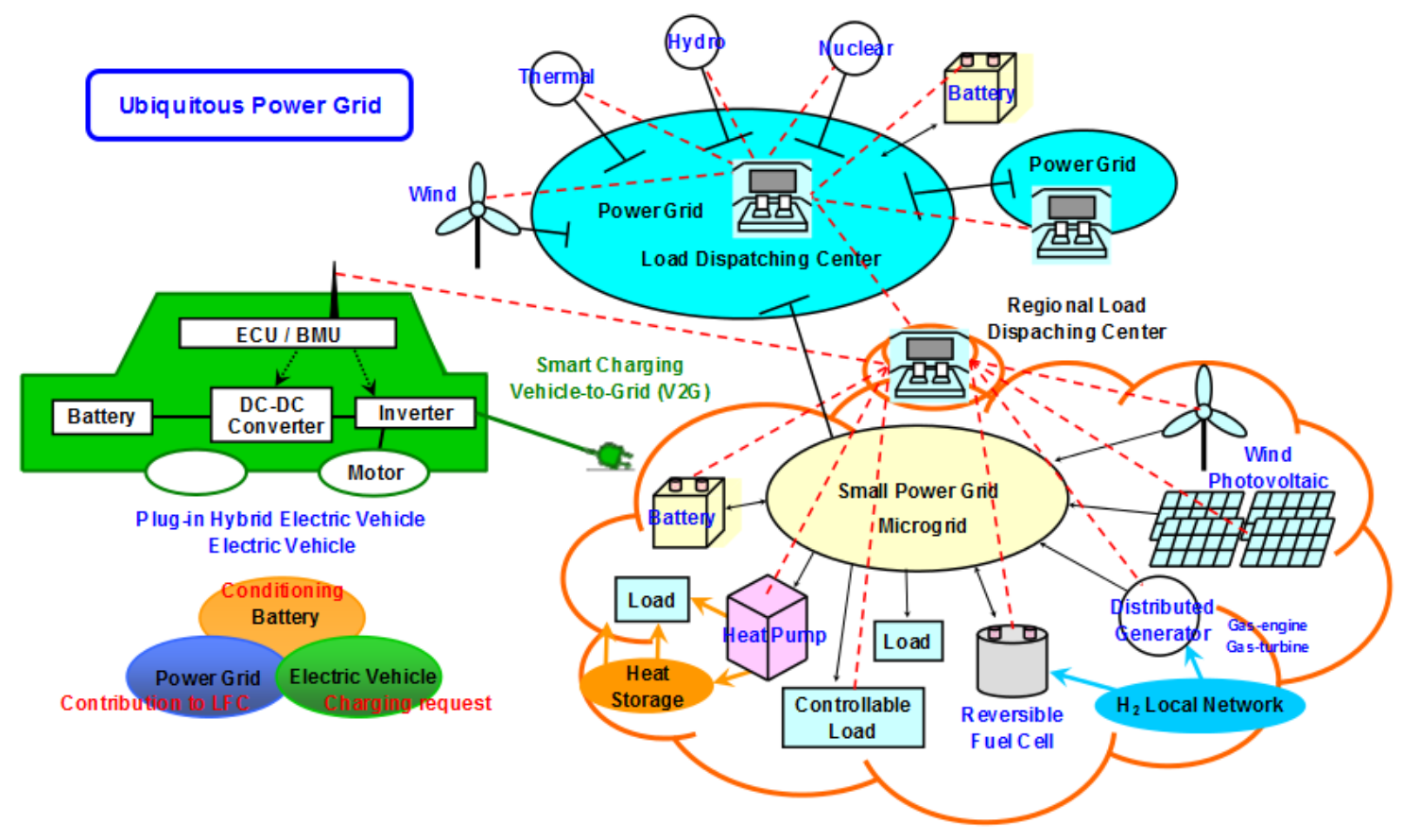

Figure 2.7: Autonomous Distributed V2G in Ubiquitous Power Grid [47]

Pursuing the concept of electrical vehicles as energy storage devices and being able to act as a power supply back to the grid, some researches study the coordination and optimization of utilizing this resource. Paper [48] considers distributed energy sources, storage devices, controllable loads, and electrical vehicles as flexible cells in the power grid. These flexible cells can be managed and coordinated to form a microgrid autonomously to achieve load balancing or other specific purposes. With the introduction of electrical vehicles, which are free to enter and leave the macrogrid, the resiliency of the macrogrid is challenged. The authors of [48] then provide their solution to the challenge. 


\subsection{Smart Grid Simulation Tools}

While all above research works achieve improvements with respect to the individual interest of study, there is no simple way of comparing the results among different researchers. Different test results are measured in different units and with respect to different quantities and come in different shapes and forms. Although most reviewed works focus on energy reduction, they vary whether the reductions are measured in terms of total energy reduction, peak load reduction, energy cost reduction, peak-to-average ratio reduction, or others. Moreover, many researchers are only focusing on single household energy efficiency improvements. How their innovations behave in the smart grid as a whole is not evaluated.

Therefore, it is thus very desirable to have a standard simulation framework which would take care of common tasks to allow users to just focus on implementing the portion of their interest of study. The simulation results would be comparable to others and would also integrate with other elements of the smart grid. Some pioneer researchers provide their solutions to this approach. For example, [49] provides a conceptual compositional simulation framework to integrate existing heterogeneous simulation models together. This framework is broken down into several layers as shown on the right side of Figure 2.8. From bottom up, the syntactical level deals with the selection or composition of different simulation models; the semantic level deals with the interaction of different simulation models at the syntactic level, such as data transfer; the scenario level contains the list(s) of simulation events to run. Unfortunately, the control level was not yet studied at the time this paper was published. While this proposed simulation framework is 
comprehensive, there are not yet much solid contents developed. There is a prototype presented in the paper, but as mentioned, the prototype is not yet complete and has several drawbacks such as limitations on data flow, inability in hierarchical use of scenarios, and lack of contents in simulation models. Overall, this proposed simulation framework is structurally promising, but still only conceptual.

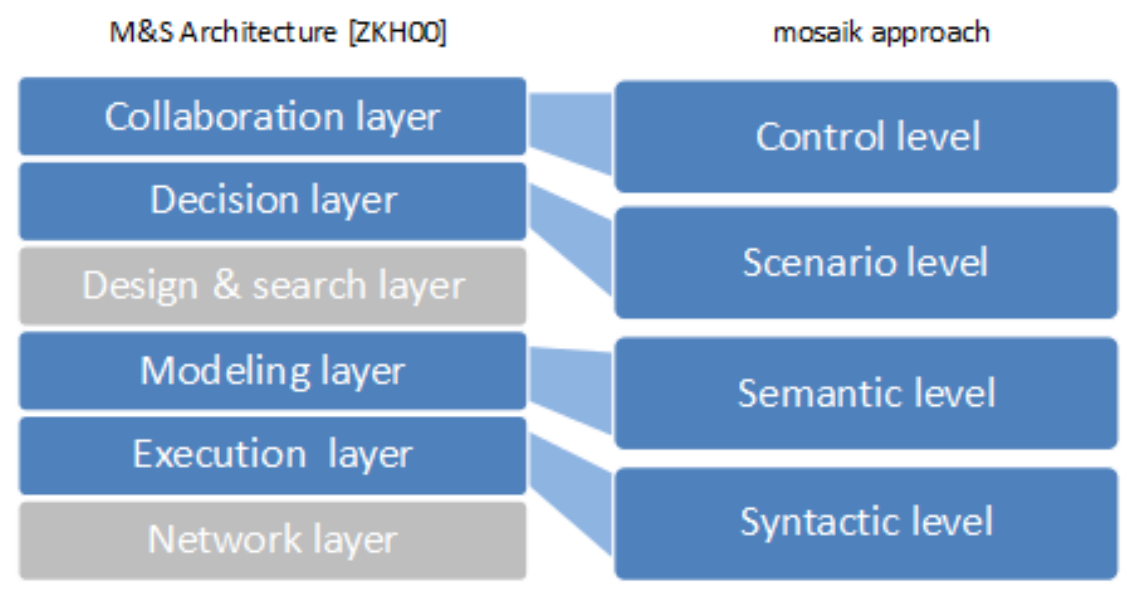

Figure 2.8: Mosaik Framework Layer Structure [49]

Several other research groups proposed their own versions of simulation frameworks. The authors in [50] proposed their design of a simulation test bed, as shown in Figure 2.9 below. The proposed test bed focuses on simulating the roles of service operation policy, network operation, market operation, and demand response. However, unfortunately again, the paper only provide a pure theoretical view of the approach. No implementation is presented in the paper. Also the test bed only considers the smart meters or AMI structure for low level communications. It may not be feasible to adapt the structure to other test environments. 


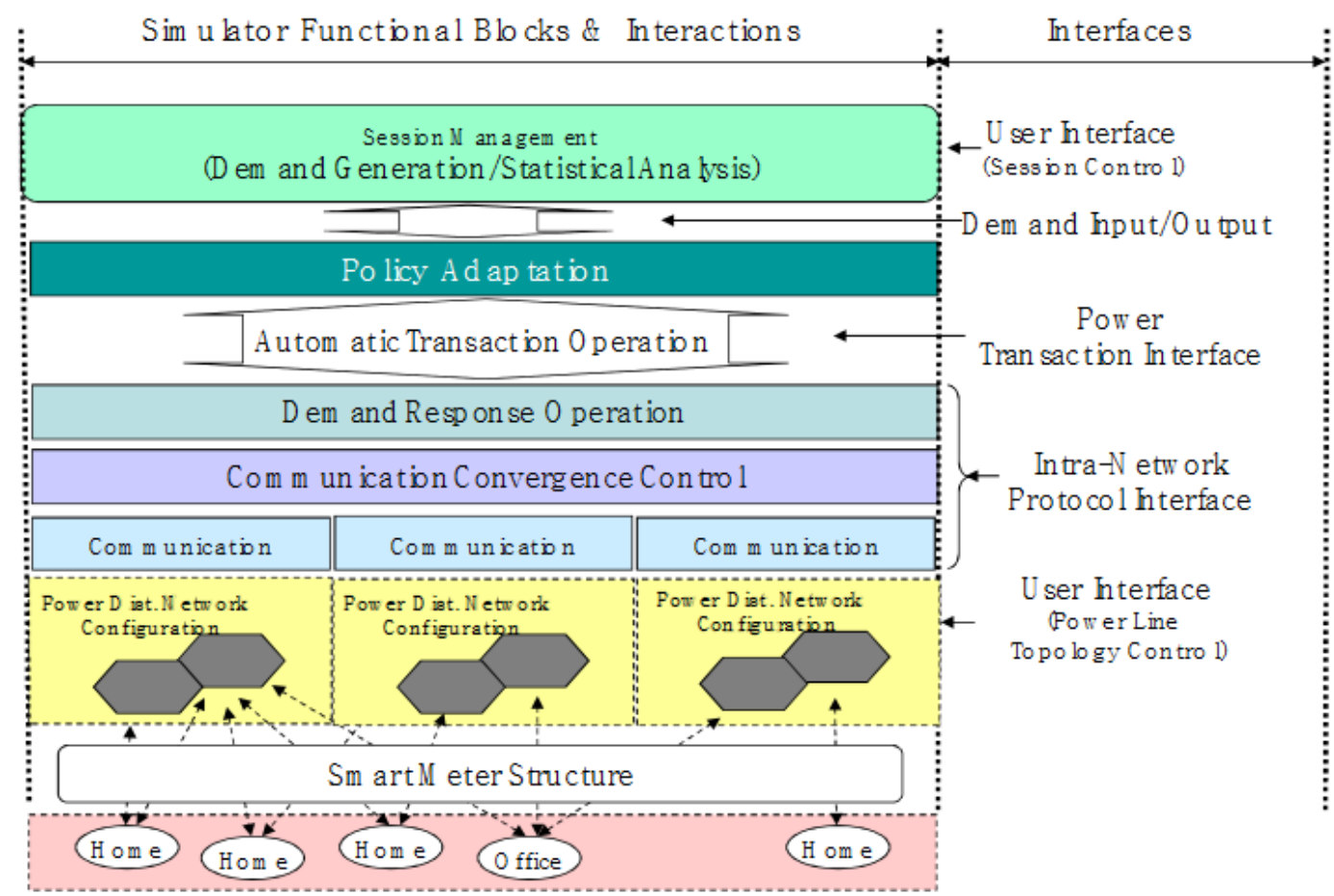

Figure 2.9: Test-bed Structure [50]

To utilize existing tools and models for a collective smart grid simulation, co-simulation method is used in some researches. Papers [51], [52], and [53] combined different tools for different simulation purposes. Some put the focus on studying the network communication technologies in smart grid, while some others focus on studying the effect of particular elements of the smart grid integration. The authors of [51] put their emphases on achieving a common communication capability among different simulation and analysis tools to achieve co-simulation. In particular, the authors worked on interconnecting the simulation flows between simulations run by Matlab/Simulink and by DIgSILENT/PowerFactory, as shown in Figure 2.10. PowerFactory is a commercial software for power grid analysis. It provides interfaces to an OPC server for Object linking for Process Control. The OPC server then provides another interface to the battery 
simulation implemented in Matlab to complete the interconnection. The proposed solution is very much centered on the simulation of battery technologies. The modeling, communication, and managing mechanisms are also built around batteries. Papers [52] and [53] both focus on studying the communication effects in smart grid applications. They both concluded that the communication delays are insignificant to cause trouble or "less than the threshold" as [53] stated.

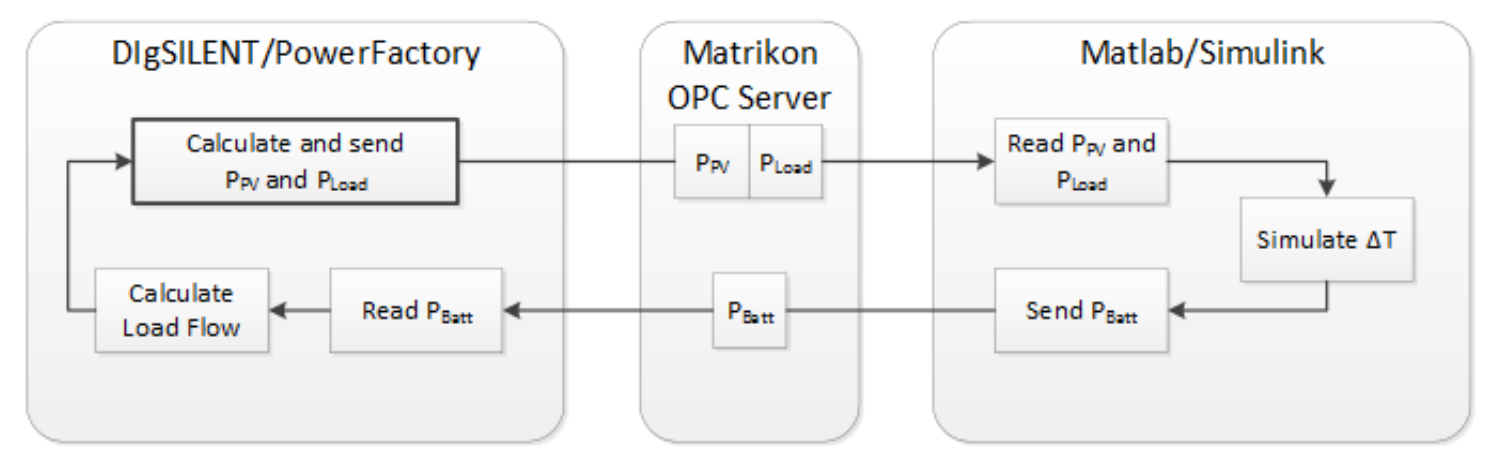

Figure 2.10: Simulation Flow Chart of [51]

Overall, the majority of existing simulation frameworks provide only conceptual views of the designs. Some proposed designs have comprehensive coverage on the included aspects in the framework. However, the complex nature of these designs causes serious obstacles in realizing the simulation platforms. These proposed frameworks are largely empty in contents and thus not ready to use. A very broad simulation scope can also sometimes imply a lack of focus. Users of such a system may need to implement the complete simulator from top to bottom to meet their specific needs. On the other hand, some proposed frameworks are very specific to certain devices or appliances simulation. These frameworks are instead not very expandable. 
In our work, we propose and implement a framework that focuses on demand side residential usage simulation, but leave it open ended with respect to the ability to integrate different components and aspects into the proposed framework. The developed simulation framework should be equipped with fundamental simulation essentials to carry out basic simulations. The design of the simulation framework should be modular for the ease of expansion. 


\section{Chapter 3: Design and Implementation}

This chapter provides an overview of the proposed simulation framework and its implementation details. High level design and the simulation framework component functionalities are discussed in Section 3.1. The implementation details and justification of the design decisions are provided in Section 3.2

\subsection{High Level Design}

\subsubsection{Design Scope and Assumptions}

First of all, it is important to understand the category of the desired end product of the design. In particular, should the design be a simulation program or simulation framework. In general, a program is a set of coded instructions that implements a specific behavior. A framework, on the other hand, is a structure or skeleton to support or guide the actual implementation of programs that expand the framework, and it defines the interactions between the programs. Various simulation programs can be implemented following the same simulation framework. Since our end goal is to define a structure to allow different implementations for different simulation purposes, our desired outcome is thus the design of a simulation framework.

Next, before talking about the actual design of the simulation framework, it is important to define the scope of the simulated world. The proposed simulation framework focuses on the demand side, more specifically the residential households' consumption. It excludes the simulation of apartment buildings and business estates or factories. However, it provides an expansion capability for those aspects to be easily integrated into the 
framework. For simplification and better comparability, the same type of household appliance consumes the same amount of energy in all households, regardless of the brand of the appliance or the size of the appliance. To randomize and thus resemble reality, different households own different number of units of the same appliance. This can be changed if a more sophisticated model is desired. While most appliances are common to all households, such as lights and refrigerator, some appliances are only owned by a subset of the population, such as electrical vehicle. The selection and rate of household ownership of these special appliances can be controlled. Furthermore, each and every appliance is categorized to be either a shiftable appliance or a none-shiftable appliance. Shiftable appliances can alter their operation time without sacrificing their functionality, while the none-shiftable appliances have to be used when they are needed. For example, a light has to be switched on immediately when one needs to enter a dark room, and thus a light is a none-shiftable appliance. On the other hand, the dishwasher can delay its operation cycle as long as it finishes its job before the next use. Therefore, a dishwasher is considered as a shiftable appliance.

\subsubsection{Design Goal}

As explained in the research objective section, the proposed simulation framework aims at providing a fundamental ability for appliance operation coordination simulation. Particular interest will be put on obtaining the average consumption/cost of energy both for each individual household and all households as a whole. Peak energy consumption is also a key aspect. When the simulation framework is fully utilized, intercommunication and large scale coordination among all enabled appliances in all simulated households 
can be achieved. Both the overall load condition on the grid and individual consumption profile are of interest in this study. Although the simulation framework is designed for this goal, only a few simple algorithms are provided to demonstrate the accuracy and flexibility of the proposed simulation framework. These algorithms first justify the use of the simulation framework, and secondly allow a researcher to observe the change in consumption behavior under different algorithms.

\subsubsection{Design Principle}

Throughout the design and implementation of the simulation framework, several general principles and guidelines were followed as listed below. These principles and guidelines together provide a foundation for a flexible and accurate framework suitable for the needs of different users.

1. Modular structure for scalability: Different components of the simulation framework can be selected and instantiated to meet individual specific requirements. New modules can also be introduced and integrated to support more functionality. The simulation framework is therefore scalable to meet different needs from different users. For example, different elements can be selected to form different households with specific characteristics such as number of occupants, dwelling types, appliance counts and etc.

2. Library structure for extension capability: Quantities, variables and models are categorized into different libraries with respect to their properties or functionalities. The libraries are extendable. Introduction of new libraries and adjusting of library entries are independent from the logical code path. 
3. Randomization for reflecting reality: Controlled randomness is applied at appropriate places to represent the diversity in the real world in order to simulate the overall grid consumption behaviors.

4. Independence from the choice of communication technology: Inter and intra communications among appliances and control stations are to be independent from the technology or standard of choice.

5. Friendly coding for better maintainability: An easily useable and maintainable coding style should be followed for both using and further coding of the simulation framework. Meaningful comments and critical error messages should be embedded to make debugging easier. Unit test should be done on every smallest functional component.

\subsubsection{Design Static View}

Figure 3.1 shows the top level block view of the main components of the simulation framework and the relationships among different function components. Descriptions and functional details of each block are summarized in Table 3.1. In general, this block diagram provides the overall structure of the framework to support various simulation program implementations. In Figure 3.1, the "Consumption Profile Generator" and the "Coordination Logic Library" are the hearts of the simulation framework. The former one houses the overall main control of the simulation program, and houses the consumption profile generation process for non-shiftable appliances. It also contains an invoker to select the desired coordinated consumption process in the "Coordination Logic Library" block. The invoked process is in charge of implementing the desired coordination 
algorithm and managing the required facility libraries. The facility libraries can be freely included/excluded with respect to individual implementations. The models depicted within each library block only represent the basic functionality they should provide. Extensions and modifications are up to individual simulation program implementation.

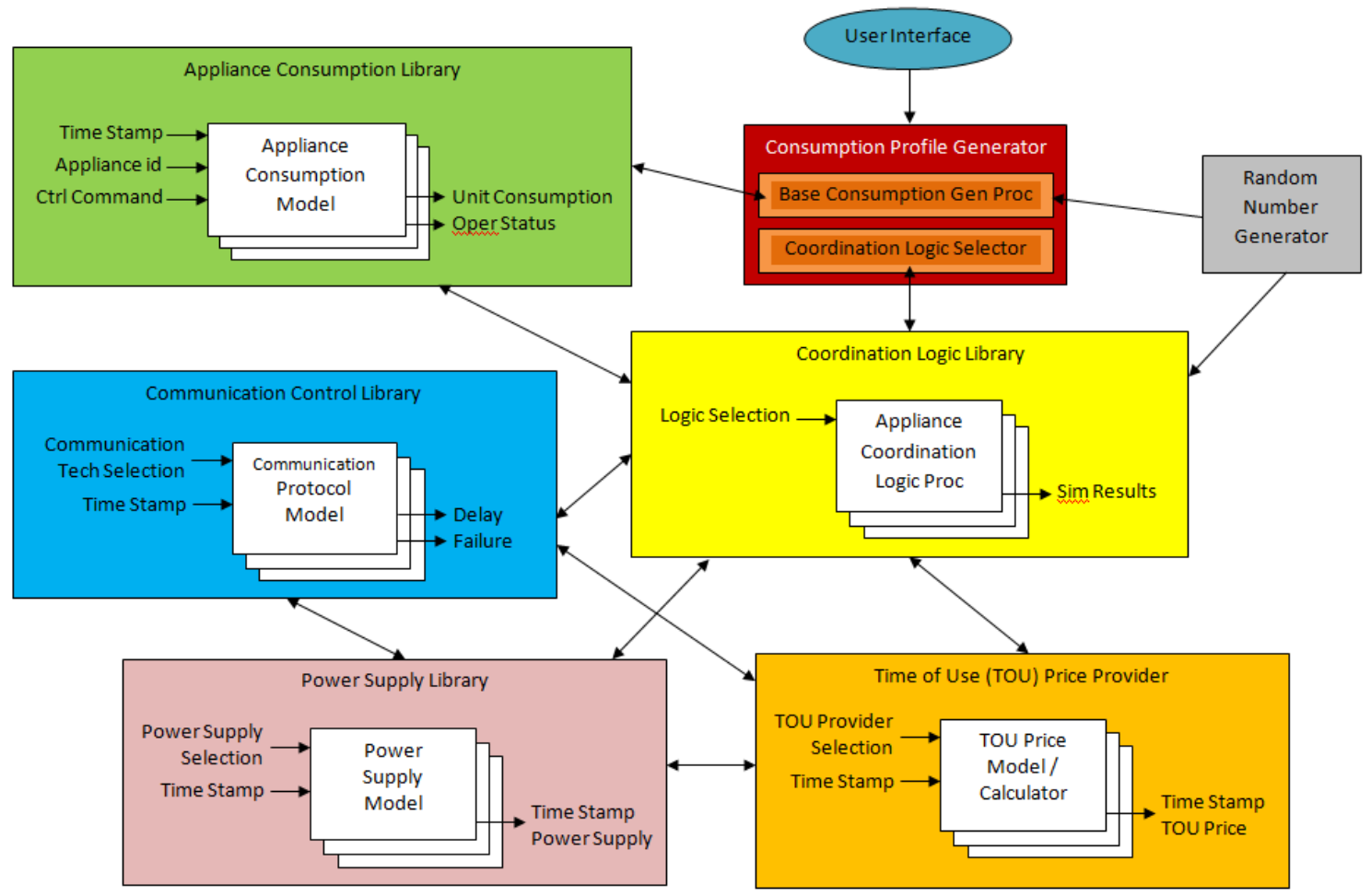

Figure 3.1: Simulation Framework Block Diagram

The block diagram illustrated in Figure 3.1 is inspired from looking at various descriptions and illustrations in different papers presented in Chapter 2. It is both a simplification/realization from theoretical designs (such as the simulation framework in [49]), and also a combination/extraction from various specific coordination studies. The end result features a simpler architecture focusing on residential appliance study, but offers flexibility for different implementations to satisfy specific purposes. Table 3.1 below provides a description of the components of the proposed framework. 
Table 3.1: Simulation Framework Block Description

\begin{tabular}{|c|c|}
\hline Block Name & Block Function \\
\hline User Interface & $\begin{array}{l}\text { This block houses the user-controlled parameters, such as the } \\
\text { number of households to simulate and which coordination } \\
\text { algorithm to use for the simulation. It also houses the main } \\
\text { program to invoke the core of the simulation. More information } \\
\text { is given in Section 3.2.1. }\end{array}$ \\
\hline $\begin{array}{c}\text { Consumption Profile } \\
\text { Generator }\end{array}$ & $\begin{array}{l}\text { This block is the core of the simulation framework. There are } \\
\text { two sequential parts to this block. The first part generates a base } \\
\text { consumption profile using none-shiftable appliances only. The } \\
\text { second part then invokes the coordination process found in the } \\
\text { "Coordination Logic Library". This block also manages the } \\
\text { simulation result report and plot. More explanations on this } \\
\text { block are given in Section 3.2.3. }\end{array}$ \\
\hline $\begin{array}{c}\text { Appliance } \\
\text { Consumption Library }\end{array}$ & $\begin{array}{l}\text { This block houses the database for appliances' consumption } \\
\text { models. Appliance consumption properties such as the hourly } \\
\text { consumption and duration of operation of each appliance are } \\
\text { captured in the model. More information on this block is given } \\
\text { in Section 3.2.2. }\end{array}$ \\
\hline $\begin{array}{l}\text { Random Number } \\
\text { Generator }\end{array}$ & $\begin{array}{l}\text { This block simply generates random numbers using a uniform } \\
\text { distribution to introduce randomness to reflect reality. More } \\
\text { details of randomization are given in Section 3.2.8. }\end{array}$ \\
\hline $\begin{array}{c}\text { Coordination Logic } \\
\text { Library }\end{array}$ & $\begin{array}{l}\text { This block contains the logic behind different coordination } \\
\text { algorithms to make decision on the operation of the shiftable } \\
\text { appliances. This block interacts with other facility library blocks } \\
\text { the most. More details on the coordination algorithms are } \\
\text { provided in Section 3.2.4. }\end{array}$ \\
\hline $\begin{array}{c}\text { Time Of Use (TOU) } \\
\text { Price Provider }\end{array}$ & $\begin{array}{l}\text { This block is in charge of providing the Time Of Use energy } \\
\text { price in real time according to the pricing scheme of choice. } \\
\text { More explanations on energy pricing schemes are given in } \\
\text { Section 3.2.5. }\end{array}$ \\
\hline $\begin{array}{l}\text { Communication } \\
\text { Control Library }\end{array}$ & $\begin{array}{l}\text { This block manages communication among various components, } \\
\text { such as how consumption is reported to the control station and } \\
\text { how the TOU prices are distributed. More information on this }\end{array}$ \\
\hline
\end{tabular}




\begin{tabular}{|l|l|}
\hline & block is given in Section 3.2.6. \\
\hline \multirow{3}{*}{ Power Supply Library } & $\begin{array}{l}\text { This library houses the communication parameters of the } \\
\text { simulation, such as the success rate of packet delivery and the } \\
\text { packet count. More information on this block is given in Section } \\
3.2 .7 .\end{array}$ \\
\hline
\end{tabular}

\subsubsection{Prototype Event Flow}

Following the definition of the proposed simulation framework, we have implemented a prototype simulation program. A typical run of the implemented simulation program is as follows. Firstly, a user sets the simulation parameters in the implementation of the "User Interface" block. The available simulation parameters are described in Table 3.3 below. Then, the main simulation program housed in the "Consumption Profile Generator" block is invoked. The main program first initiates and instantiates objects according to the simulation parameters. For example, it makes reservation in memory for the variables that are to be used in the simulation according to how many households are included in the simulation. Also, household appliances selection is done in this stage. For example, the program will remove electrical vehicles from households if the electrical vehicles are excluded in the simulation set by the user. After the program initiation, the main program then launches the base consumption generation process. The implementation details of this process are described in Section 3.2.3. This process basically takes the none-shiftable appliances in the "Appliance Consumption Libaray" to lay down a base consumption profile. Next, the main program invokes the targeted coordinated consumption generation process housed in the "Coordination Logic Library". This coordination process first modifies and/or configures the related aspects specific to the coordination method. For 
example, if a coordination scheme is to schedule the appliance's operation based on time, this process will modify the appliance consumption model instances to reflect change. Then, at each time stamp, specific operations will be performed based on the logics of individual coordination schemes. At this stage, the other three facility libraries (“Communication Control Library", "Power Supply Library", and "Time of Use (TOU) Provider" libraries) are selectively associated. For example, if no coordination is involved (treated as one type of coordination algorithm which simply does not contain a real logic), none of other three facility libraries are used. With another example, if a coordination algorithm is based on making decisions to respond to the TOU price changes, then the "Time of Use (TOU) Provider" library and the "Communication Control Library" are used. Due to lack of a suitable power supply side of model, the "Power Supply Library" is not used in the current implementation of the simulation program prototype. Lastly, after the end of coordinated consumption generation, the main program takes back the control and generates a simulation report and plot.

\subsubsection{Simulation Prototype Outputs}

This section describes the outputs provided at the end of the simulation from the implemented prototype based on the proposed framework. This should aid as a guide and example for all simulation program implementations. In general, the program keeps a copy of all the raw data generated from the simulation. The simulation is 24-hour based; it starts at midnight and ends at midnight the next day. This is most useful to see the detailed effects of coordination algorithms across a day. This is also a common usage as seen in multiple papers mentioned in Chapter 2. Longer simulation window (such as a 
week) can be seen as an array of individual simulations, or can be modified at individual implementations to incorporate some properties such as the home occupant weekly working pattern. The user of the simulation prototype has control of the sample size (denoted to be $M$ ) and step size (denoted as $N$ ) of the simulation. The sample size represents the number of households to include in the simulation, and the step size measures how many evaluation points exist within the 24 hours time frame. For example, if 100 households are simulated and computations are done every minute, then the $M^{*} N$ matrix size will be $100 * 1440$. Having this defined, the following table summarizes the major raw data kept by the simulation in the current implementation.

Table 3.2: Simulation Raw Data Table

\begin{tabular}{|c|c|}
\hline Category & Raw Data Kept \\
\hline \multirow{4}{*}{ Consumption } & $\begin{array}{l}{[1 * N] \text { Pre-generated reference total consumption profile with }} \\
\text { no coordination involved. }\end{array}$ \\
\hline & $\begin{array}{l}{\left[M^{*} N\right] \text { Current simulation consumption profiles generated for }} \\
\text { each household. }\end{array}$ \\
\hline & $\begin{array}{l}{[1 * N] \text { Actual total consumption profile from the current }} \\
\text { simulation. }\end{array}$ \\
\hline & $\begin{array}{l}{\left[1^{*} N\right] \text { Reported total consumption known at the control }} \\
\text { station from the current simulation. This may vary from the } \\
\text { actual profile due to communication errors. }\end{array}$ \\
\hline \multirow{3}{*}{ TOU } & $\begin{array}{l}{\left[1^{*} N\right] \text { Pre-generated reference TOU price profile linearly }} \\
\text { proportional to the reference total consumption profile with } \\
\text { no coordination involved. }\end{array}$ \\
\hline & $\begin{array}{l}{\left[M^{*} N\right] \text { TOU price profiles seen at each household from the }} \\
\text { current simulation. }\end{array}$ \\
\hline & $\begin{array}{l}{\left[1^{*} N\right] \text { Actual TOU price profile calculated at the control }} \\
\text { center using the actual total consumption profile generated } \\
\text { from the current simulation. }\end{array}$ \\
\hline
\end{tabular}




\begin{tabular}{|c|l|}
\hline \multirow{2}{*}{ Communication } & $\begin{array}{l}{\left[1^{*} N\right] \text { Reported TOU price profile calculated at the control }} \\
\text { center using the reported total consumption profile generated } \\
\text { from the current simulation. }\end{array}$ \\
\cline { 2 - 3 } & $\begin{array}{l}{\left[1^{*} N\right] \text { An OnPeak-MidPeak-OffPeak pricing TOU profile that }} \\
\text { is used in real life as another reference. }\end{array}$ \\
\hline \multirow{7}{*}{$\begin{array}{l}{\left[1^{*} K\right] \text { Packet counts for the total packet sent with each }} \\
\text { communication technology used in the current simulation. } \\
\text { tere } K \text { represents the number of different communication } \\
\text { communication used. For example, for simplicity, each } \\
\text { control center uses another technology, then } K=2 .\end{array}$} \\
\cline { 2 - 3 } & {$\left[M^{*} K\right]$ Duration of erroneous communication periods. } \\
\hline
\end{tabular}

From this raw data, the simulation generates a report to show results such as daily energy cost and standard deviations. It also generates a graph along with the report at the end of each simulation. These are illustrated in Chapter 4.

\subsection{Design Details}

In this section, the design details of the implemented simulation prototype program based on the proposed simulation framework is presented. The design details should aid as both explanations of the inner workings of the framework, and also a guideline or example for expansion to meet different simulation purposes.

\subsubsection{User Interface}

User interface is the one-stop place for users to set all the parameters for the simulation. This block is only associated with the "Consumption Profile Generator" block. It invokes 
the main program of the simulation. The table below lists the currently available parameters that are implemented.

Table 3.3: Implemented User Interfaces

\begin{tabular}{|c|c|c|c|}
\hline Parameter & Type & & Description \\
\hline simSize & Integer & \multicolumn{2}{|c|}{$\begin{array}{l}\text { This is the sample size }(M) \text { as mentioned in Section 3.1.6. It } \\
\text { represents the number of households to include in the } \\
\text { simulation. (i.e. } 100 \text { means } 100 \text { households). }\end{array}$} \\
\hline timeSlots & Integer & \multicolumn{2}{|c|}{$\begin{array}{l}\text { This is the step size }(N) \text { as mentioned in Section } 3.1 .6 \text {. It } \\
\text { measures how many evaluation points exist within a } 24 \\
\text { hours time frame. (i.e. } 24 * 60 \text { means evaluation is done } \\
\text { every minute). }\end{array}$} \\
\hline EVSim & True/False & \multicolumn{2}{|c|}{$\begin{array}{l}\text { This parameter defines whether to include electric vehicles } \\
\text { in the simulation. A value of true means that electric } \\
\text { vehicles will be included in the simulation. }\end{array}$} \\
\hline EVPercent & Percentage & \multicolumn{2}{|c|}{$\begin{array}{l}\text { This parameter sets up the percentage of households that } \\
\text { own an electric vehicle. This parameter is effective only } \\
\text { when EVSim is set to true ( } 1 / 20 \text { means one in } 20 \\
\text { households owns an electric vehicle). }\end{array}$} \\
\hline \multirow[t]{6}{*}{ coordMethod } & \multirow[t]{6}{*}{ Choice } & \multicolumn{2}{|c|}{$\begin{array}{l}\text { This parameter defines the coordination algorithm to use in } \\
\text { the simulation. Below we list the currently available } \\
\text { choices that are implemented; more information is given in } \\
\text { Section 3.2.4. }\end{array}$} \\
\hline & & Choice & Description \\
\hline & & Basic & $\begin{array}{l}\text { Only None-shiftable appliances are } \\
\text { simulated. }\end{array}$ \\
\hline & & Traditional & $\begin{array}{l}\text { Shiftable appliances are uncoordinated. } \\
\text { None-shiftable appliances are included. }\end{array}$ \\
\hline & & Programmable & $\begin{array}{l}\text { Shiftable appliances are scheduled } \\
\text { based on the time of a day. None- } \\
\text { shiftable appliances are included. }\end{array}$ \\
\hline & & Threshold & 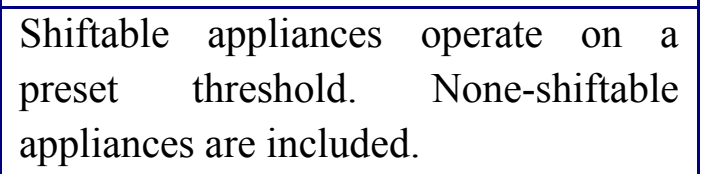 \\
\hline
\end{tabular}




\begin{tabular}{|c|c|c|c|}
\hline \multirow[t]{7}{*}{ TOUChoice } & \multirow[t]{7}{*}{ Choice } & \multicolumn{2}{|c|}{$\begin{array}{l}\text { This parameter defines the time of use pricing scheme to } \\
\text { use in the simulation. Below we list the currently available } \\
\text { choices that are implemented. More information is given in } \\
\text { Section 3.2.5. }\end{array}$} \\
\hline & & Choice & Description \\
\hline & & FlatRate & $\begin{array}{l}\text { Electricity price throughout the day is } \\
\text { constant. }\end{array}$ \\
\hline & & ThreeLevel & $\begin{array}{l}\text { Based on three levels: Off_Peak, } \\
\text { Mid_Peak, On_Peak. }\end{array}$ \\
\hline & & Historical & A reference pre-recorded TOU profile. \\
\hline & & DayAhead & $\begin{array}{l}\text { A known price profile recorded from } \\
\text { the previous day. }\end{array}$ \\
\hline & & Linear & $\begin{array}{l}\text { TOU price is linear between highest } \\
\text { and lowest price limit. }\end{array}$ \\
\hline $\begin{array}{l}\text { Consumption- } \\
\text { Report- } \\
\text { Choice }\end{array}$ & Choice & \multicolumn{2}{|c|}{$\begin{array}{l}\text { This parameter defines the communication technology of } \\
\text { choice for the household consumption report. Currently, } \\
\text { two methods are implemented: Pushing and Polling. }\end{array}$} \\
\hline $\begin{array}{l}\text { Consumption- } \\
\text { Report- } \\
\text { Cycle }\end{array}$ & Integer & \multicolumn{2}{|c|}{$\begin{array}{l}\text { This parameter defines how often the consumption is } \\
\text { reported to the control center. For example, a value of } 15 \\
\text { means consumptions are reported every } 15 \text { time steps, or } 15 \\
\text { minutes if timeslots is defined as } 24 * 60 \text {. }\end{array}$} \\
\hline $\begin{array}{l}\text { Consumption- } \\
\text { Report- } \\
\text { SuccessRate }\end{array}$ & Percentage & \multicolumn{2}{|c|}{$\begin{array}{l}\text { This parameter represents the packet delivery success rate } \\
\text { for consumption reporting expressed in percentage. For } \\
\text { example, a value of } 0.95 \text { means that } 95 \text { percent of the } \\
\text { packets will be delivered successfully to the control center. }\end{array}$} \\
\hline $\begin{array}{l}\text { TOU- } \\
\text { Report- } \\
\text { Choice }\end{array}$ & Choice & \multicolumn{2}{|c|}{$\begin{array}{l}\text { This parameter defines the communication technology of } \\
\text { choice for TOU price distribution from the control center. } \\
\text { Two options are currently available: Broadcast and } \\
\text { Request. }\end{array}$} \\
\hline $\begin{array}{c}\text { TOU- } \\
\text { Report- } \\
\text { SuccessRate }\end{array}$ & Percentage & \multicolumn{2}{|c|}{$\begin{array}{l}\text { This parameter represents the packet delivery success rate } \\
\text { for TOU price distribution expressed in percentage. For } \\
\text { example, a value of } 0.95 \text { means that } 95 \text { percent of packets } \\
\text { are delivered successfully to each household. }\end{array}$} \\
\hline blackOutSim & True/False & \multicolumn{2}{|c|}{$\begin{array}{l}\text { This parameter represents whether to include a } \\
\text { communication blackout in the simulation or not. A value }\end{array}$} \\
\hline
\end{tabular}




\begin{tabular}{|c|l|l|}
\hline & & $\begin{array}{l}\text { of true will simulate communication blackout. A typical } \\
\text { example of a communication blackout could happen due to } \\
\text { a tower failure. }\end{array}$ \\
\hline blackOutStart & Integer & $\begin{array}{l}\text { This parameter sets the starting time for the blackout to } \\
\text { occur in terms of a time stamp. This parameter is effective } \\
\text { only when blackOutSim is set to True. For example, a value } \\
\text { of } 15 * 60 \text { means the blackout will start at 3:00pm. }\end{array}$ \\
\hline blackOutEnd & Integer & $\begin{array}{l}\text { This parameter sets the ending time for the blackout to stop } \\
\text { in terms of a time stamp. This parameter is effective only } \\
\text { when blackOutSim is set to True. A value of } 23 * 60 \text { means } \\
\text { the blackout ends at 11:00pm. }\end{array}$ \\
\hline $\begin{array}{c}\text { Blackout- } \\
\text { Percentage }\end{array}$ & Percentage & $\begin{array}{l}\text { This parameter defines the percentage of all simulated } \\
\text { households which are affected by the blackout. For } \\
\text { example, a value of } 0.3 \text { means that } 30 \text { percent of all } \\
\text { simulated households will be affected by the blackout. }\end{array}$ \\
\hline
\end{tabular}

\subsubsection{Appliance Consumption Library}

The appliance consumption library houses the appliance consumption models to simulation their consumption properties. The appliance consumption models should basically provide unit time consumptions with respect to the appliance type. It takes three parameters as the input to the model, namingly Time Stamp, Appliance ID, and Control Command. The former two inputs are obvious with their meaning. The Control Command input here simply mean to turn the appliance on or off for simplicity. As for the output of the model, Unit Time Consumption and Operation Status are provided. This "Appliance Consumption Library" block interacts with the "Consumption Profile Generator" block and the "Coordination Logic Library" block. The associated blocks 
basically instantiate instances of the appliance consumption models with respect to specific configurations.

There are two major types of models in this library and a total of three parts representing three categories of household appliances. Recall that any household appliance is categorized to be either a shiftable appliance or a none-shiftable appliance. Thus, the two basic types represent these two groups respectively. In the first type, the none-shiftable appliances are further broken down into two parts. The first part is called the "Time Constrained None-Shiftable Appliances" table. This part of the model contains the entries for none-shiftable appliances that make most sense to only operate during specific times in a day, in general situations. For example, it makes most sense to use a stove only for cooking dinner around $5: 00 \mathrm{pm}$ to $7: 00 \mathrm{pm}$, and thus a stove is listed in the "Time Constrained None-Shiftable Appliances" table. On the other hand, a stove does not have to operate only in the evening. Different people use the same type of appliance differently. Therefore, the "Random Access None-Shiftable Appliances" model is introduced to bring some randomness to the appliances usages. A given appliance can be part of both categories of none-shiftable appliances. The main difference between these two categories arises from their values set in the table entries, which are explained in more details in subsequent sections. Lastly, for the shiftable appliances, although their operations are dependent on the coordination method of choice, the consumption model still contains their typical usage without any coordination. The consumption models are coded in a table format. Table 3.4 demonstrates an example of the appliance consumption 
models with respect to three types of appliances. Please see Appendix A for the actual implementation of the full table.

Table 3.4: Appliance Consumption Table Example

\begin{tabular}{|c|c|c|c|c|c|c|c|c|}
\hline Appliance & $\begin{array}{l}\text { In Use } \\
\text { (bool) }\end{array}$ & $\begin{array}{c}\text { Time } \\
\text { Constraint } \\
\text { (Min index) }\end{array}$ & $\begin{array}{c}\text { Time } \\
\text { Constraint } \\
\text { (Max index) }\end{array}$ & $\begin{array}{l}\text { Consumption } \\
\text { (W/min) }\end{array}$ & $\begin{array}{l}\text { Duration } \\
\text { (minute) }\end{array}$ & $\begin{array}{c}\text { Remainder } \\
\text { (minute) }\end{array}$ & $\begin{array}{c}\text { Use } \\
\text { Frequency } \\
\text { (/day) }\end{array}$ & $\begin{array}{c}\text { Odds Of } \\
\text { Using } \\
(\%)\end{array}$ \\
\hline \multicolumn{9}{|c|}{ Time Constrained None-Shiftable Appliances } \\
\hline Stove & True/False & 1020 & 1140 & 20.86 & 45 & 45 & 1 & $1 / 120$ \\
\hline \multicolumn{9}{|c|}{ Random Access None-Shiftable Appliances } \\
\hline Stove & True/False & 1 & 1440 & 20.86 & 15 & 15 & 2 & $2 / 1440$ \\
\hline \multicolumn{9}{|c|}{ Shiftable Appliances } \\
\hline Dishwasher & True/False & 1200 & 1440 & 20 & 90 & 90 & 1 & $1 / 120$ \\
\hline
\end{tabular}

*Note: The timeSlot simulation parameter used in this table is $24 * 60=1440$ (minutes)

When the simulation program starts, each household is assigned a copy of their own consumption table instance. The table attributes, as shown above, are applicable to individual household. Each row in the table corresponds to a single appliance. While the meaning of "Appliance" and "In Use" are quite obvious, others are explained in more detail in the following subsections.

\subsubsection{Time Constraint Attributes}

The min and max (or start and end) indexes in the appliance consumption table together define the time constraint for the applied appliance to start its operation. The unit of these time constraints is in terms of the time slots (see the note of Table 3.4). Each time slot represents one minute in the given example, and thus the $1020^{\text {th }}$ time slot represents 17:00 $(1020=17 * 60)$. The applied appliance can start its operation at any time within this time constrain in a random fashion controlled by the "Odds of Using" attribute which is explained later. For example, as shown in Table 3.4, the dishwasher is constrained to start its operation any time between 20:00 $(1200=20 * 60)$ and 24:00 $(1440=24 * 60)$. The logic 
behind this time constraint on the dishwasher is that dishwashers are usually used after dinner time before going to bed.

Observe the differences on these time constraint for a stove under the "Time Constrained None-Shiftable Appliances" versus the "Random Access None-Shiftable Appliances". The random access one basically has the freedom to start its operation any time in a day, but with a lower chance to actually being used.

\subsubsection{Consumption Attribute}

Consumption attribute provides the average/typical power consumption of a type of appliance. The unit of this attribute is in watt/timeslot. In Table 3.4, since one time slot represents one minute, the unit is thus in fact Watt/min. The calculation for this attribute is to divide the universal $\mathrm{kWh}$ measurement by the timeSlot simulation parameter. The $\mathrm{kWh}$ consumption measurements are obtained from a few different sources. Governmental publications such as the "Energy Consumption of Major Household Appliances Shipped in Canada, Trends for 1990-2009" [54] [55] from Natural Resource Canada provides trustworthy data. Some online sites such as [56] and [57] have good summaries and good explanations on how different appliances are used. Consumer electronics retailer websites provide product specs for a variety of brands, types, and sizes. Finally, my personal usages are verified using the Energy Display provided by the local utility "peaksaverPLUS" program [58]. All these data are brought together to complete the appliance consumption table of the simulation framework. 


\subsubsection{Duration, Remainder, and Use Frequency Attributes}

The duration attribute defines the typical operation time lapse of an appliance, in terms of time slots. This information is gathered from both the sources mentioned in Section 3.2.2.2 and a small size survey among colleagues and friends. The result may not be accurate but does serve the purpose of the simulation. While the duration is a constant value for each entry, the remainders attribute decreases once an appliance is started. The remainder attribute represents the time left until the end of duration, also expressed in terms of time slots. For simplicity, once an appliance starts its operation, it will not stop until it reaches the end of the duration. Upon finishing the operation cycle of an appliance, the simulation looks into the Use Frequency attribute to see if such appliance can be used again. If the value in the Use Frequency is greater than 0 , the value in Duration is copied to the Remainder field for the next use. Therefore, the Use Frequency represents the reoccurrences allowable for the same appliance to operate in the same day.

\subsubsection{Odds of Using Attribute}

This last attribute in the appliance consumption table introduces randomness into the appliance operations. It is a percentage value representing the probability that an appliance can start its operation. It is easiest to understand this probability value as the allowable operation count per time period. For example, a value of $1 / 120$ means the applied appliance is allowed to start its operation only once within 120 time slots. The formula for this probability is defined in Equation 3.1. 


\section{Equation 3.1:}

$$
\text { Odds of Using }=\frac{\text { Use Frequency }}{\text { Time Constraint (Max index })- \text { Time Constraint (Min index })}
$$

With the introduction of this randomness, appliances across different households get turned on at random times within the time constraint, in order to simulate the randomness of when different people return from work for example. Also some appliances do not get turned on at all to simulate the cases that either the household does not own that particular appliance, or that a household does not use that appliance within that time period.

The Time Constraints (min \& max), Duration, Remainder, Use Frequency and Odds Of Using attributes differentiate time-constrained none-shiftable appliances from their random access counterparts. In general, time-constrained appliances are more important and model regular behaviors, while the random access ones model the exceptions or rarer behaviors. Typically, time-constrained appliances have higher probability to be used to re-enforce their importance.

\subsubsection{Consumption Profile Generator}

The consumption profile generator houses the main program of the simulation. It controls the simulation process. The consumption profile generator first instantiates class objects and initiates simulation parameters according to the user settings. The most important instantiations are the copies of the appliance consumption library objects for each household. The generator customizes each consumption library object. For example, it 
will remove electrical vehicles from the object if EVSim is set to false. At this stage, the generator also reserves memory space for the entries in Table 3.2.

After the initializing phase, the consumption profile generator starts the actual simulation phase. There are two sequential stages to this phase. The first stage is called the "base consumption generation" and the second stage is called the "coordinated consumption generation". The first stage generates a set of base consumption profiles for each household using only the none-shiftable appliances. Coordination algorithms do not apply to none-shiftable appliances, and thus no communication is required among them. Therefore, the consumption generation algorithm for this stage is simple. A time-driven simulation technique is used for the generator. A variable is used to record the time of the simulation, and it is incremented with a fixed time step. Operation and decisions on whether appliances can be turned on are made at every time slot for every household. The pseudo logic is presented in Figure 3.2 below.

for each household

for each timeslot

for each appliance

check if the appliance is already in use

if yes, update its consumption

if no, figure out if it should get turned on :

check if Use Frequency $>0$

check if within the time constraint

roll a dice to see if < Odds of Using

if all passed, turn the appliance on

Figure 3.2: Base Consumption Generation Pseudo Logic 
The reason a time-driven simulation is chosen over an event-driven approach is that the consumption behaviors of appliances over a period of time are time-based continuous activities. Although time is modeled as discrete time steps, at each step certain events are bound to happen at either the power supply side or the power demand side. Simple examples are continues charge on electricity usage on the power supply side, and alwayson appliance consumptions such as from a fridge on the demand side. Therefore, time is the driving force of the simulation, and events happen at each time step. Also, a centralized time-driven approach does not need to deal with possible asynchronous behaviors posed by an event-driven technique. Overall, a time-driven simulation is the natural and obvious approach for the proposed simulation framework.

The second stage of the appliance consumption generation involves different coordination logics. Different coordination methods may need different configurations and modifications to the instantiated objects. Therefore, a separation component block is used to represent the coordination logic library as shown in Section 3.2.4 below.

\subsubsection{Coordination Algorithm Library}

Coordination algorithm library is used by the appliance consumption generation procedure with regard to the shiftable appliances. It houses the complete process and controls with respect to coordinated consumption generation. Therefore a high level model of this library can be seen as only have one input and one output. The input is used to select which coordination logic to use, and the output is the simulation results. The coordination logics make decision on the operation of controlled appliances. It updates 
the consumption profiles on top of the base consumption profiles described in Section 3.2.3. The implementations of individual coordination logics are unique. The following subsections give more details.

\subsubsection{Traditional Appliances Consumption Generation}

This first logic in fact has no coordination involved. It simulates how none-smart-grid enabled appliances (i.e. traditional appliances) are used. This type of appliances does not have knowledge of TOU information nor do they exchange information with any other appliances. They are turned on physically whenever they are needed. Therefore, there is no reconfiguration or modifications needed. Also there is no association to the “Communication Control Library", "Power Supply Library", and "Time of Use (TOU) Price Provider" blocks. The purpose for the implementation of this category is to first verify the accuracy of the implemented simulation framework, and second to set a baseline for comparisons with other coordination logics. All none-shiftable appliances operate using the same logic depicted in Figure 3.2.

\subsubsection{Programmable Appliances Consumption Generation}

Programmable appliances do not directly coordinate their operation with control stations or with other appliances. However, they have the ability to schedule their operations based on the time of a day and sometimes they even have the capability to learn the usage pattern from individual home owners [31]. An example of this type of appliance is the programmable thermostat. Users have the ability to schedule the temperature profile they want, and some newer ones have the ability to learn a users' schedule. While no 
sophisticated learning algorithm is implemented, the scheduling mechanism of the programmable appliances is implemented. It is done by modifying the shiftable appliance consumption table for each household according to a user-defined schedule. After the modifications, the new consumption tables contain the scheduled behaviors. What remains is to use the same logic depicted in Figure 3.2 to update the consumptions. Again, there is no association to the other three facility library blocks.

\subsubsection{Price Threshold Controlled Appliance Consumption Generation}

This type of appliances is representative of true smart-grid-enabled technology. The operations of the price threshold controlled appliances can be delayed until the time of use price, sent from the control station or utility, meets the user set price threshold. The communication in this case is on a per-household basis. There is no appliance to appliance communication or appliance to control station communication. The pseudo logic behind this type of coordination is slightly different from the base consumption generation logic shown in Figure 3.2. It has to perform one more check before turning on an appliance. Please refer to the figure below to see an illustration. 


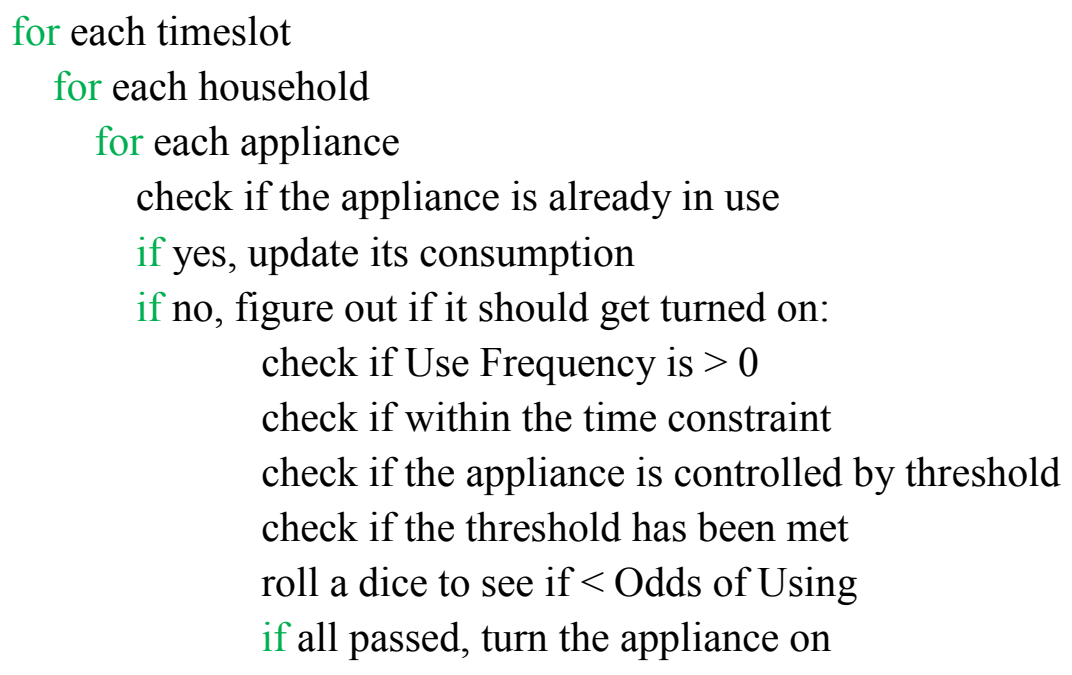

Figure 3.3: Threshold Controlled Appliance Consumption Generation Pseudo Logic

With this threshold controlled coordination method, two facility libraries are used. The "Communication Control Library" provides control to TOU price distribution and household consumption reporting. For example, at one time stamp, household consumption calculated in the "Application Coordination Logic Process" is ready to get reported to the control center. It invokes the "Communication Protocol Model" instance, which has already been instantiated when the coordination method is selected. In the implemented example, there is no real communication protocol is modeled. The only results are whether the communication failed or delayed. After that, the consumption value is sent to the "TOU Price Model/Calculator". In this case, since there is no power supply model implemented, the TOU price is calculated solely based on the total consumption. The calculated TOU price is then again sent back to the coordination process via the communication control module to complete one cycle of the simulation at one time stamp. 


\subsubsection{Time of Use (TOU) Price Provider}

TOU prices with respect to a user's choice of pricing scheme is provided either by loading the stored pricing profiles or by built in calculation mechanism, in real time during the simulation. When loading from a file, a user needs to match the data size to the timeSlot simulation parameter. The method of producing the TOU prices is selected by the "Appliance Coordination Logic Process". The TOU prices are distributed at every chosen time stamp regardless of the choice of pricing scheme. Failures in distribution can be simulated and this is discussed later in Section 3.2.6.

The current implementation provides five different pricing schemes. The "FlatRate" scheme prices the energy usage throughout the day with the same constant rate that is set by the user. "Historical" and "DayAhead" schemes both load a pricing profile from a prerecorded text file and use that profile to determine prices in the simulation. While the "Historical" scheme loads an average price profile that is generated from the simulation framework itself, the "DayAhead" scheme actually gets its data from a utility company [59]. The "ThreeLevel" scheme is also currently being used by another utility company,

as shown in the figure below. Due to its simplicity, where prices are not dynamically changing, this scheme is being hard-coded into the pricing scheme library. 


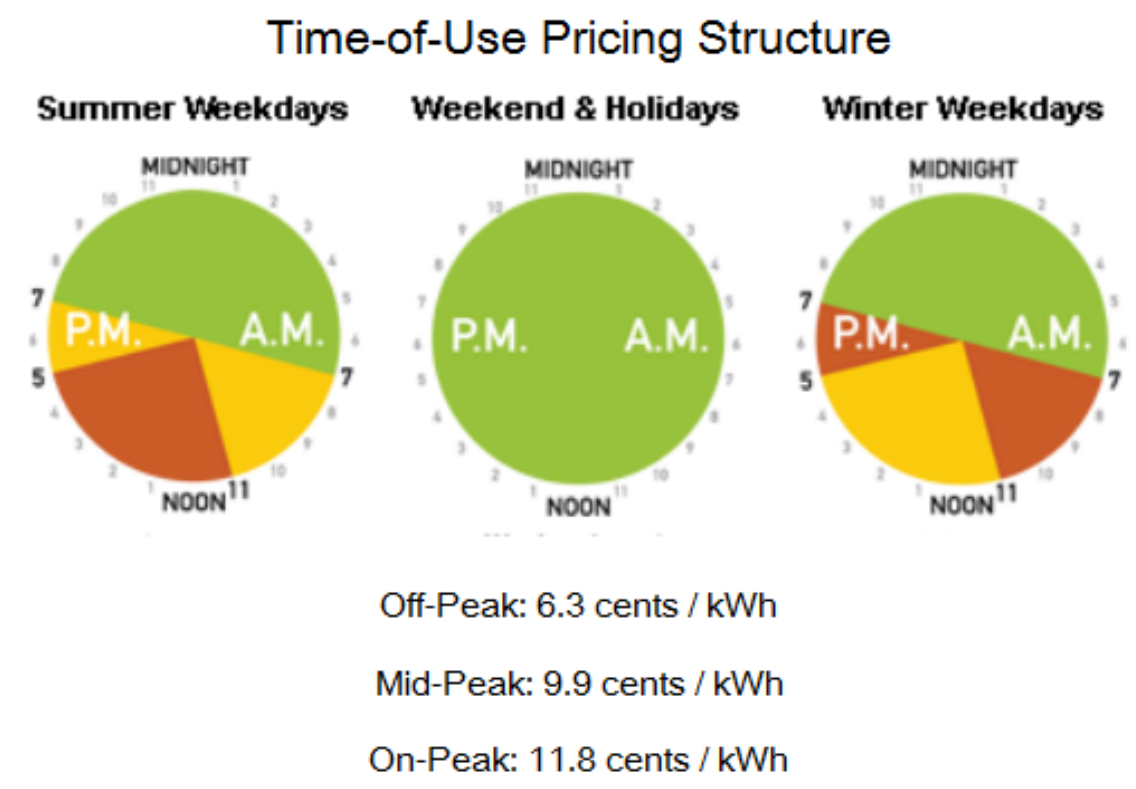

Figure 3.4: HydroOttawa TOU Pricing Structure [60]

The last implemented pricing scheme is called "Linear" pricing. This is an example of a dynamic pricing scheme. The TOU price is calculated at every evaluation timestamp and is linearly proportional to the consumption or available power supply. Again, there was no real power supply model been implemented in the prototype, we used the total consumption in the calculation. The minimum $\left(C_{\min }\right)$ and maximum $\left(C_{\max }\right)$ total consumption are first obtained from historical simulations. Users then define the minimum charge $\left(P_{\min }\right)$ and the maximum charge $\left(P_{\max }\right)$. They are currently being defaulted to be the Off-Peak price and the On-Peak price shown in Figure 3.4. However these are free to change and the points in between scales accordingly. With the measured total consumption $\left(C_{T O U}\right)$ at the evaluation timestamp, the corresponding TOU price is calculated using Equation 3.2. 
Equation 3.2:

$$
P_{\text {TOU }}=\frac{P_{\text {max }}-P_{\text {min }}}{C_{\max }-C_{\min }} *\left(C_{T O U}-C_{\min }\right)+P_{\text {min }}
$$

\subsubsection{Communication Control Library}

The communication control Library deals with inter and intra communications of the simulation framework. In the implemented prototype, it simulates the logical behavior of communication, but it abstracts away from the technical details of communication protocols. The "Communication Protocol Model" illustrated in Figure 3.1 only provides a very basic model to real communication protocols. This is because the interest of the proposed simulation framework focuses on the behavioral level of simulation instead of the micro scale inner workings of specific protocols, as explained before. Therefore, the most interested outputs of the communication model is to indicate whether the communication failed at a given time stamp. We did also implement a delay function in the communication model. However the delay time unit is simulation time slots. However, details of specific communication protocols can be modeled and stored in the library. Also, an external simulation tool, or built in simulation facilities in Matlab can be connected for better simulation.

With respect to a threshold controlled operation example, a user can define the average packet success rate for each communication method as mentioned in Table 3.3. Here a simple model is used to represent the coordination system. It is assumed that all households report their power consumptions to one central control station, with a single packet each time. The control station calculates the TOU price at each time stamp based 
on the received consumption reports. The calculated TOU price is then distributed to all households via broadcasting. For simplicity, there is no packet retransmission mechanism implemented. Once a per-household consumption reporting packet is lost, the control center would not have the information of that particular household for that time stamp. Likewise, once a TOU price distribution packet is lost, the household(s) would not have the up-to-date price. Instead, the price from the last update is used again. Also, regional communication failure simulation can be simulated. User can set up the error-ed time frame and affected household percentage. The effects of these failures are demonstrated in Chapter 5.

\subsubsection{Power Supply Library}

The power supply Library houses the models to simulation the power availability status in the simulation. The stored models can be a pre-recorded power supply profile, or actual simulation models of power generators. Renew able power generation models are a good example to fit in this library. The envisioned "Power Supply Model" should provide unit time power supply at given time stamp. However, unfortunately, there was no real implementation has been done with the prototype, and thus this block was not instantiated. This block is obviously associated with the "Time of Use (TOU) Price Provider" block for TOU price calculation. Also it is associated with the "Coordination Logic Library" and the "Communication Control Library" blocks. A simple example for the need of association is dynamic control of power generation. The coordination process has the knowledge of the snapshot of the power supply and consumption at a time stamp. It can 
decide whether more power is needed to be generated, and communicate the decision to the power generation model via the communication control module.

\subsubsection{Levels of Randomization}

As demonstrated in previous sections, randomizations are employed in many places with the aim to recreate the varying and bursty behavioral patterns observed in reality. Different households are equipped with different appliances in the simulation; the same appliances are used at different times of the day and communication failures can happen at random times. All these randomizations are using a uniform distribution in which all the possible numbers have equal odds to appear. In reality, the probabilities are sometimes skewed. For example, a stove is more probable to be used at breakfast time and dinner time compared to other time such as at night and away from home time periods. Therefore, a selectable probability control mechanism is implemented. As shown in the figure below, a sinusoidal wave is designed to peak at around 7:00am and 7:00pm (x-axis). The peak and valley magnitudes of the wave are adjustable and currently set at 1.2 and 0.6. When this wave is multiplied with the original Odds of Using attribute of each appliance, it manipulates the probability accordingly. For example, the probability of usage is increased by $20 \%$ around 7:00am, and the probability is decreased by $20 \%$ around 2:00am. 


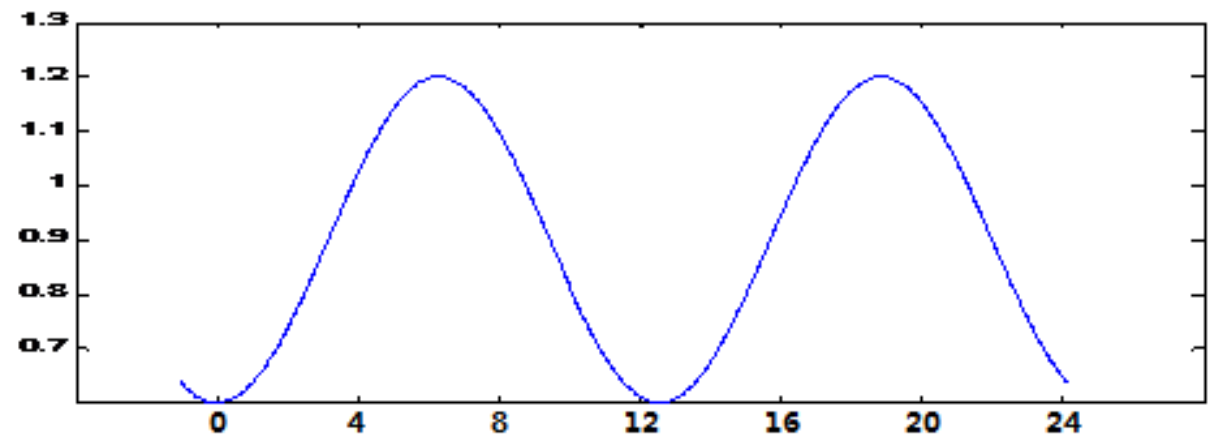

Figure 3.3: Probability Control Mechanism

\subsubsection{End of the Day Consumption Loopback}

The designed simulation framework is 24 -hour based. It does not have knowledge of the previous day or the next day. A problem caused by this is the limitation of the consumption continuity in the simulation across two days. For example, if an appliance is turned on before the end of the day and keeps operating into the next day, the consumptions in the second day is not within the scope of the 24-hour view. To solve this problem, a loopback of the consumptions for unfinished operations back to the beginning of the day is employed. The following figure provides a visual illustration of this mechanism.

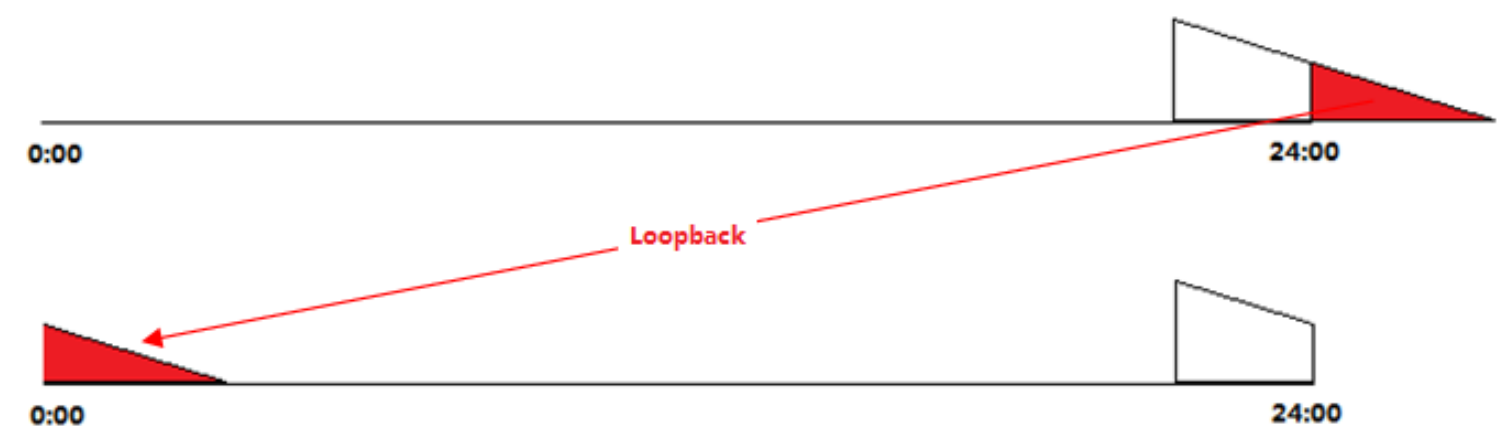

Figure 3.4: Example of Consumption Loopback 
As shown in Figure 3.8, the triangle represents a rundown timer of an appliance, and the red portion of the triangle represents the operation of the appliance that lasts into the next day. The loopback operation basically takes the red portion of the triangle and puts it back to the beginning of the day. This enhances accuracy of the simulation in light of the assumption that households' behavior during two consecutive days would be very similar. 


\section{Chapter 4: Results and Validation}

This chapter presents some initial simulation results and findings with no appliance coordination employed. Discussions and validations are provided on the simulation results. This chapter provides a ground of comparison for coordination algorithms simulations that will be presented in the next chapter.

\subsection{Simulation Environment}

All the simulations presented in this thesis are modeled based on the North America region. It is assumed that all simulated households are independent dwellings such as detached houses or town houses. Each household owns their private appliances and provides their own daily essentials such as hot water and space heating or cooling. Since the simulated environment is set to be the summer season, air conditioners are the active appliances instead of space heaters. For simplicity, the same type of appliance (such as a TV or fridge) consumes the same amount of energy among all households. What differs is the amount of these types of appliances owned by each household, which is generated by the randomization process explained in Section 3.2.8. All the simplifications assumed here can be refined and extended in the proposed simulation framework with further study.

The simulations are performed with Matlab. Matlab provides easy code execution with no compilation needed. A debugger is also integrated in the software which makes runtime value monitoring possible. Values of simulation variables can also be printed out or viewed in internal windows at any time. Manipulations and mathematical operations of 
the data are available both before and after the simulation. All these features make the execution of a simulation and the analysis of its results very simple.

A personal laptop is used as the carrier of the simulations. It has a quad core processor with $1.6 \mathrm{GHz}$ of processing power. $4 \mathrm{~GB}$ of built-in RAM is shared among all running applications. Although Matlab does support multi-threading and parallel processing, its capability of utilizing those technologies is still limited. Certain more advanced features need a specific toolbox or patch to support. The simulation speed may thus not be optimized. Also, Matlab is known to be slower than $\mathrm{C} / \mathrm{C}++$. On average a simulation with 1000 households takes around two hours to run. However, the limitations on the speed of the simulation can be improved by using a faster machine and/or some effort on simulation optimization. A metropolitan-scale simulation can also be broken down to regional representations. Overall, the simulation environment is relatively easy to use. However, future work might be needed to improve the simulation speed to scale to larger scenarios.

\subsection{Simulation Result Illustration}

As briefly mentioned in Chapter 3, a report and a plot are automatically generated at the end of each simulation. The plot provides visual demonstration and comparison among consumption profiles, and the report summarizes the plot and highlights some key parameters. Figure 4.1 shows a typical plot generated by the simulation framework. The figure displays the simulation plot for 1000 households that undergo a traditional operation without having their appliances coordinated. The plot is composed of an upper 
subplot and a lower subplot. The upper subplot displays the consumption aspect of the profiles while the lower subplot displays the TOU price aspect of the profiles.
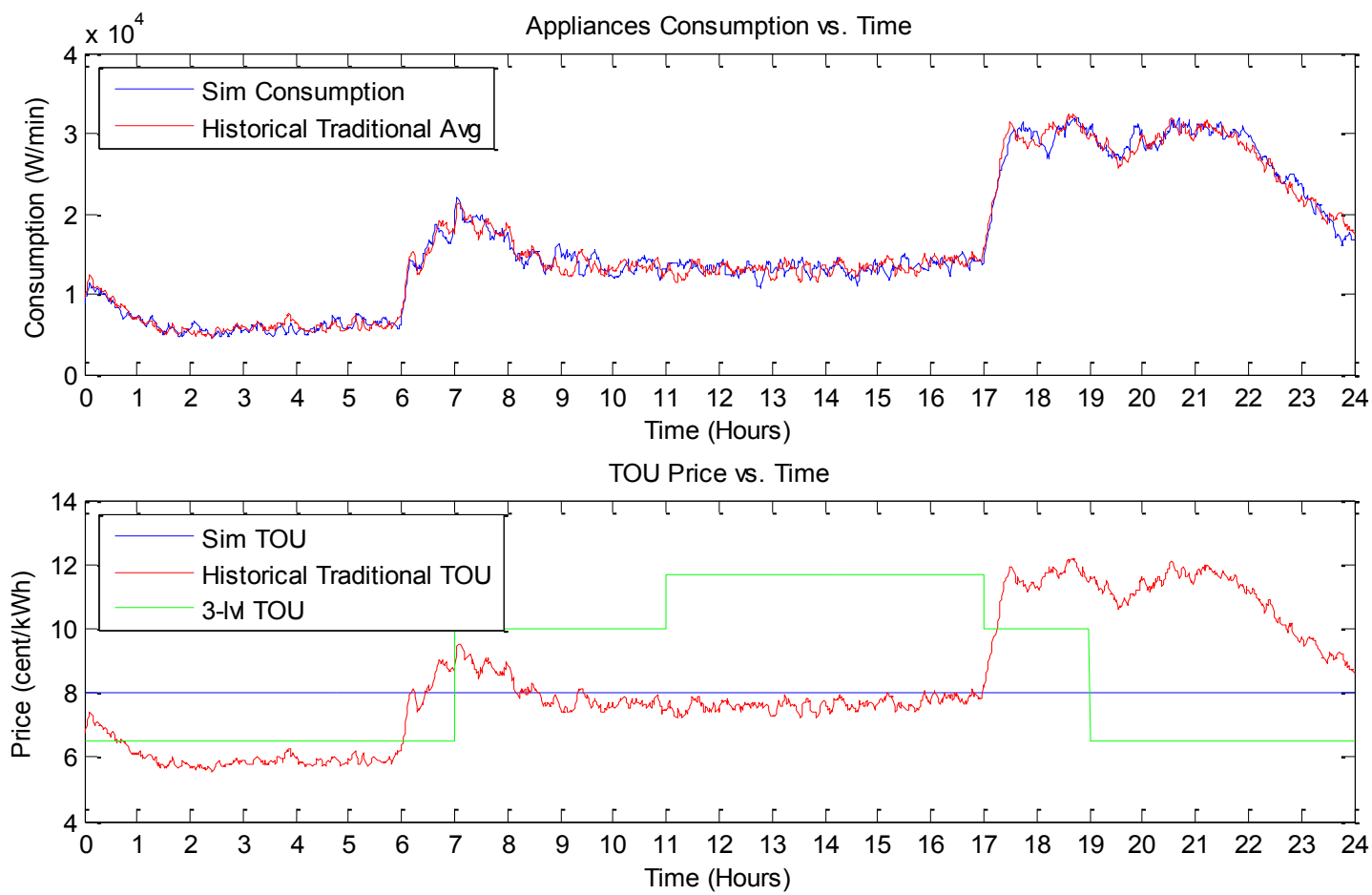

Figure 4.1: Sample Simulation Plot

For the upper subplot, it displays two consumption profiles. The blue line is the consumption generated from the current simulation; whereas the red line is a prerecorded consumption average of 1000 households for reference purposes. The reference line is used in all simulations as a comparison line. It has undergone tTest several times to verify there is no significant change compared to other simulations with identical settings. Hence the reference line is able to represent average or typical traditional consumption behavior. The blue line is subject to change according to each simulation, whereas the red line remains the same for all simulation results. The $\mathrm{x}$-axis represents the time stamp of a day. For simplicity, the unit is displayed in hours, although the actual 
data is sampled at every minute in the simulation. The y-axis represents the power consumption measured in Watt/minute. This unit is used instead of the standard unit $(\mathrm{kW} / \mathrm{h})$ because the simulation is configured to perform calculations every minute. It can be converted to the standard unit simply by multiplying with $\left(\frac{60}{1000}\right)$.

The lower subplot of Figure 4.1 contains the TOU price profile information. There are three lines in this subplot, two of which are for reference purposes. The blue line is the actual TOU profile used/generated from the current simulation according to the choice of TOU pricing scheme. In this example, the Flat Rate pricing scheme is used and the flat price is set to $8 \mathrm{cent} / \mathrm{kWh}$, thus a straight blue line is displayed in the figure. The second TOU price profile is represented by the green line. It is equivalent to the Summer Weekday OnPeak-MidPeak-OffPeak pricing scheme depicted in Figure 3.5, except for the price values. The price values displayed in Figure 3.5 are for Winter Weekdays, whereas the simulation is configured to deal with summer consumption. Therefore, the actual price represented by the green line reads: Off-peak $=6.5 \mathrm{cent} / \mathrm{kWh}$; Mid-peak $=10$ cent $/ \mathrm{kWh}$; and On-peak $=11.7 \mathrm{cent} / \mathrm{kWh}$. As shown in Figure 4.1, the staircase-shaped green line peaks from 11:00am to 5:00pm, during the hottest period of a day when air conditioners are working the hardest. The price drops down to off-peak after 7:00pm because the temperature starts to drop at that time. The last TOU price profile displayed in the lower subplot of Figure 4.1 is the red line. The red line is a linearly proportioned price profile with respect to the reference average consumption line (red line) shown in the upper half of the figure. The values of this red price line are calculated with Equation 3.2. It symbolizes the dynamic value of energy at different points of time for residential 
households' usages. For example, the higher the value is, the more precious the energy is at that moment of time because of high demand, and vice versa. Both the red line and the green line are for reference purposes and do not change from simulation to simulation.

Beside the plot discussed above, each simulation also generates a simulation report as shown in Figure 4.2. There are two parts to the report, separated by the "Simulation Report" line. The first part is a status report to indicate the current operation performed by the simulation. It comes especially handy when performing a large simulation. It does not only indicate simulation progress, but also records the elapsed time for each stage of the simulation. At the "Simulation Initializing" stage, user-defined parameters are read by the simulation framework and memory reservations are made based on the settings. After that, class objects are instantiated in the "Simulation Starting" stage. The appliance consumption table instance for each household is randomized according to the simulation settings. For example, electrical vehicle entries are removed from the table if EV is set to be excluded in the simulation settings. Having all the preparations finished, the actual consumption profile generation starts at the "None-shiftable Appliance Consumption Generation Running" stage. The base consumption profiles for each household discussed in Section 3.2.3 are generated in this stage. Followed by that, the shiftable appliances' consumptions according to different coordination algorithms are generated in the "Shiftable Appliance Consumption Generation Running" stage. Lastly, after the completion of the data generation, the simulation report and plot are generated in the "Simulation End" stage. 
04-Mar-2013 23:07:13: Simulation Initializing...

04-Mar-2013 23:07:13: Simulation Starting...

04-Mar-2013 23:07:14: None-shiftable Appliances Consumption Generation Running...

04-Mar-2013 23:36:22: Shiftable Appliances Consumption Generation Running...

05-Mar-2013 00:10:16: Simulation End

$======================$ Simulation Report $=======================$

Number of households simulated: $\quad 1000$

Coordination method used: $\quad$ Traditional Consumption Simulation

TOU price scheme used: $\quad$ Flat Rate Pricing

Average household daily consumption: $\quad 23.08 \pm 0.34 \mathrm{kWh}$

Reference average daily consumption: $\quad 23.07 \pm 0.33 \mathrm{kWh}$

Lowest household daily consumption: $\quad 10.01 \mathrm{kWh}$

Highest household daily consumption: $\quad 36.47 \mathrm{kWh}$

Average household daily cost: $\quad 1.85 \pm 0.03$ dollars

Reference average daily cost: $\quad 1.85 \pm 0.03$ dollars

Lowest household daily cost: $\quad 0.80$ dollars

Highest household daily cost: $\quad 2.92$ dollars

Peak daily total consumption: $\quad 1916.07 \mathrm{~kW} / \mathrm{h}$ occurred at $21: 41$

Reference peak consumption: $\quad 1940.09 \mathrm{~kW} / \mathrm{h}$ occurred at 19:41

Total consumption statistics: Mean: 961.47 Standard Deviation: 499.26

Reference consumption statistics: $\quad$ Mean: 961.43; Standard Deviation: 494.4343

Figure 4.2: Sample Simulation Report 
The second part of the report summarizes some key results of the simulation. The descriptions of each quantity are given in the table below.

Table 4.1: Simulation Report Description Table

\begin{tabular}{|c|c|}
\hline Name & Description \\
\hline $\begin{array}{c}\text { Number of } \\
\text { households } \\
\text { simulated }\end{array}$ & $\begin{array}{l}\text { Reiterate the simSize parameter that has been set for the simulation. It } \\
\text { indicates how many households have been included in the simulation. }\end{array}$ \\
\hline $\begin{array}{l}\text { Coordination } \\
\text { method used }\end{array}$ & $\begin{array}{l}\text { Reiterate the coordMethod parameter that has been set for the simulation. } \\
\text { It indicates the coordination method used for the simulation. }\end{array}$ \\
\hline $\begin{array}{l}\text { TOU price } \\
\text { scheme used }\end{array}$ & $\begin{array}{l}\text { Reiterate the TOUChoice parameter that has been set for the simulation. } \\
\text { It indicates the pricing scheme used for the simulation. }\end{array}$ \\
\hline $\begin{array}{l}\text { Average } \\
\text { household } \\
\text { daily } \\
\text { consumption }\end{array}$ & $\begin{array}{l}\text { The average daily total consumption among simulated households. It is } \\
\text { calculated with the equation below, where } C_{h / \text { timestamp denotes the }} \\
\text { household consumption at each evaluated time stamp. } \\
\text { Equation 4.1: } \\
\qquad \frac{\sum_{1}^{\operatorname{simSize}}\left(\sum_{1}^{\text {timeSlots }}\left(\mathrm{C}_{\mathrm{h} / \text { timeStamp }}\right)\right)}{\operatorname{simSize}} \\
\text { The average is followed by the } 95 \% \text { confidence interval around the } \\
\text { average. It is generated by a Matlab built-in function "ttest()". The } \\
\text { confidence interval is used to indicate the reliability of the average, and } \\
\text { used to indicate the significance in difference of different samples. }\end{array}$ \\
\hline $\begin{array}{l}\text { Reference } \\
\text { average daily } \\
\text { consumption }\end{array}$ & $\begin{array}{l}\text { The average daily total consumption calculated from the pre-recorded } \\
\text { reference consumption including the } 95 \% \text { confidence interval. The } \\
\text { previous description also applies to this parameter. }\end{array}$ \\
\hline
\end{tabular}




\begin{tabular}{|c|c|}
\hline $\begin{array}{l}\text { Lowest } \\
\text { household } \\
\text { daily } \\
\text { consumption }\end{array}$ & $\begin{array}{l}\text { The lowest household daily total consumption in the current simulation, } \\
\text { which is denoted with the following equation. } \\
\text { Equation 4.2: } \\
\qquad \operatorname{Min}_{1}^{\text {simSize }}\left(\sum_{1}^{\text {timeSlots }}\left(C_{\text {h/timeStamp }}\right)\right)\end{array}$ \\
\hline $\begin{array}{l}\text { Highest } \\
\text { household } \\
\text { daily } \\
\text { consumption }\end{array}$ & $\begin{array}{l}\text { The highest household daily total consumption in the current simulation, } \\
\text { which is denoted with the following equation. } \\
\text { Equation 4.3: } \\
\qquad \operatorname{Max}_{1}^{\text {simSize }}\left(\sum_{1}^{\text {timeSlots }}\left(C_{\mathrm{h} / \text { timeStamp }}\right)\right)\end{array}$ \\
\hline $\begin{array}{l}\text { Average } \\
\text { household } \\
\text { daily cost }\end{array}$ & $\begin{array}{l}\text { The average household daily electricity cost for the current simulation. It } \\
\text { is calculated with the equation below, where } T O U_{\text {timestamp }} \text { denotes the time } \\
\text { of use electricity price at each time stamp. } \\
\text { Equation 4.4: } \\
\qquad \frac{\sum_{1}^{\operatorname{sim} S i z e}\left(\sum_{1}^{\text {timeSlots }}\left(\mathrm{TOU}_{\text {timeStamp }} * \mathrm{C}_{\mathrm{h} / \text { timeStamp }}\right)\right)}{\text { SimSize }} \\
\text { It also comes with the } 95 \% \text { confidence interval around the average. }\end{array}$ \\
\hline $\begin{array}{l}\text { Reference } \\
\text { average daily } \\
\text { cost }\end{array}$ & $\begin{array}{l}\text { The average household daily electricity cost calculated with the pre- } \\
\text { recorded reference consumption profile and the TOU pricing choice } \\
\text { selected for the current simulation. The above equation and description } \\
\text { applies for this parameter too. }\end{array}$ \\
\hline $\begin{array}{c}\text { Lowest } \\
\text { household } \\
\text { daily cost }\end{array}$ & $\begin{array}{l}\text { The lowest household daily electricity cost found in the current } \\
\text { simulation. It is represented by the equation below: } \\
\text { Equation 4.5: } \\
\qquad \operatorname{Min}_{1}^{\text {simSize }}\left(\sum_{1}^{\text {timeslots }}\left(\text { TOU }_{\text {timeStamp }} * C_{\mathrm{h} / \text { timeStamp }}\right)\right)\end{array}$ \\
\hline
\end{tabular}




\begin{tabular}{|c|c|}
\hline $\begin{array}{l}\text { Highest } \\
\text { household } \\
\text { daily cost }\end{array}$ & $\begin{array}{l}\text { The highest household daily electricity cost found in the current } \\
\text { simulation. It is represented by the equation below: } \\
\text { Equation 4.6: } \\
\qquad \operatorname{Max}_{1}^{\text {simSize }}\left(\sum_{1}^{\text {timeSlots }}\left(\mathrm{TOU}_{\text {timeStamp }} * \mathrm{C}_{\mathrm{h} / \text { timeStamp }}\right)\right)\end{array}$ \\
\hline $\begin{array}{l}\text { Peak daily } \\
\text { total } \\
\text { consumption }\end{array}$ & $\begin{array}{l}\text { The maximum total electricity consumption used by all simulated } \\
\text { households at a particular time of a day. It reflects the peak load } \\
\text { requirement that power stations need to cover. The formula for finding } \\
\text { the peak value is as follows: } \\
\text { Equation 4.7: } \\
\qquad \operatorname{Max}_{1}^{\text {timeSlots }}\left(\sum_{1}^{\text {simSize }}\left(\mathrm{C}_{\mathrm{h} / \text { timeStamp }}\right)\right)\end{array}$ \\
\hline $\begin{array}{l}\text { Reference } \\
\text { peak } \\
\text { consumption }\end{array}$ & $\begin{array}{l}\text { The peak total consumption seen on the reference pre-recorded } \\
\text { consumption profile. This quantity is particularly useful to determine } \\
\text { whether a certain coordination method has the capability of reducing the } \\
\text { peak load needed from the power grid. Equation } 4.7 \text { is also employed for } \\
\text { this calculation. }\end{array}$ \\
\hline $\begin{array}{l}\text { Mean \& } \\
\text { Standard } \\
\text { deviation for } \\
\quad \text { total } \\
\text { consumption } \\
\text { per time unit }\end{array}$ & $\begin{array}{l}\text { The standard probability distribution measurements to evaluate the } \\
\text { dispersion of the data set. } \\
\text { - Mean is the arithmetic mean of the sampled data set. It represents } \\
\text { the mathematical average without any weight or any manipulation } \\
\text { on the raw data. } \\
\text { - Standard Deviation indicates how close a set of data points is with } \\
\text { respect to the mean. It is calculated by taking the square root of } \\
\text { the variance. } \\
\text { These two measurements are provided with Matlab's built-in functions } \\
\text { "mean()" and "std()". These measurements are important criteria for load } \\
\text { balancing study. }\end{array}$ \\
\hline $\begin{array}{l}\text { Reference } \\
\text { consumption } \\
\text { statistics }\end{array}$ & $\begin{array}{l}\text { The same probability distribution measurements described above that are } \\
\text { obtained from the reference pre-recorded consumption profile. }\end{array}$ \\
\hline
\end{tabular}


The items shown in the report can be freely added, moved around, or completely removed when seen fit. Each individual item can be manipulated or enhanced to suit a specific purpose of study.

\subsection{Result Validation and Verification}

First of all, it is important to understand the difference between validation and verification. Validation is about whether the designed product does provide the desired functionality to meet the requirements or the purpose of building the product; verification, on the other hand, is about whether the end product implements the desired functionalities correctly. With respect to this thesis work, validation is about whether the designed simulation framework is able to satisfy the research objective; and verification is then about whether the implemented simulation prototype is able to generate appliance consumptions correctly.

Let us first talk about the validation of the simulation framework. As explained in Section 1.2 , the research objective is to develop a flexible simulation framework to study the behaviors and impacts of smart grid enabled household appliances. There are a few key elements in such a statement. First, the design should be a flexible simulation framework. This statement is satisfied by the implementation of the simulation program prototype that realizes three different kinds of appliance coordination algorithms by following the same framework. Explanations and instructions of how to utilize different framework components for different simulation program implementations are presented throughout

Chapter 3. The second key element is the involvement of smart grid. Two key aspects of 
smart grid are incorporated in the proposed simulation framework. First, smart grid is a hybrid of electricity power grid and network communication grid. This aspect is obviously embodied by the "Communication Control Library", "Time of Use (TOU) Price Provider" and the "Coordination Logic Library" components of the proposed simulation framework. Second aspect of smart grid is the control of power flow. Again, such aspect is covered by upper three components plus the "Power Supply Library" block itself. Renewable energy generation models and models to interpret electrical vehicles as an energy source can be implemented in this block. The last key element of the objective is the focus of residential appliances. The "Appliance Consumption Library" is built around household appliances, instead of commercial machineries. The coordination logics are operated on the residential appliances as well. Overall, the proposed simulation framework satisfies the desired functionalities defined in the research objective. Therefore the framework is validated.

Next, let us talk about verification. Firstly, white box unit tests were performed manually and with the integrated debug tool throughout the coding phase. Tests were performed at a smallest functional basis, such as verification upon each consumption library instance modification. Expected values were verified against different possible configurations. Boundary tests were performed and error messages were included where necessary. A debug option was embedded in the code, which, upon activation, will output critical traces and values during the simulation. The following sections illustrate the result from integration tests and final results. 
With the simulation plot and report explained in the previous section, this section further provides a verification of the accuracy of the simulation results. First, let us take a look at the shape of the consumption profile. As observed from Figure 4.1, there are two consumption peaks in a day for household usages. One smaller peak happens around 7:00am, and another larger peak happens starting at 5:00pm and lasts through the evening. The reason for the peaks is obvious, the morning peak occurs when people prepare their breakfast with some low-consumption appliances such as toaster and microwave; the evening peak is due to households' evening activities involving some major appliances such as stove, dishwasher and TV. Energy consumption for the rest of the time is mainly due to always-on appliances such as fridge and air conditioner, and standby power consumptions for some idle appliances. There is also some random usage of all kinds of appliance throughout the day as mentioned in Chapter 3. To compare to an actual recording of household usages, the following figure provides a daily consumption profile record averaged across a year in Northern Ireland [61]. Unfortunately, there is no similar North American plot that we found at the time of research. However, the general consumption behavior is similar.

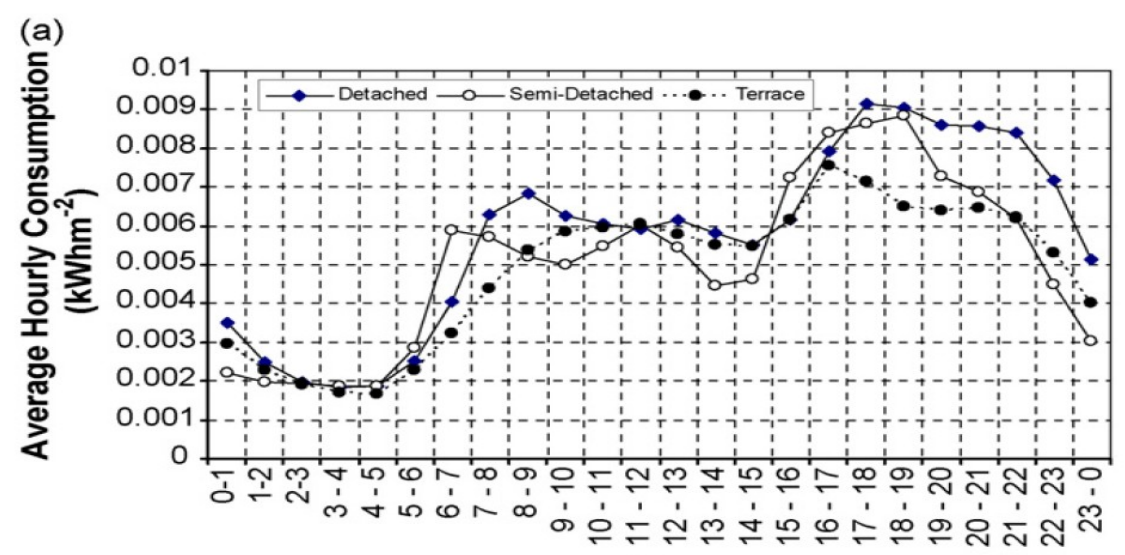

Hour

Figure 4.3: Average Daily Annual Electricity Consumption for Different House Types [63] 
The figure plots the average daily consumption profiles of three different types of houses, which are detached houses, semi-detached houses and terrace units. As shown in the figure, the general shapes of all three types of households closely resemble the simulation result shown in Figure 4.1. The biggest difference is the smoothness and gradualness of the lines. The actual recorded values shown in Figure 4.3 form smooth curves with gentle ramping up and down, whereas the simulation result displaces wavy curves and sudden rises of the energy consumption around 6:00am and $5: 00 \mathrm{pm}$. The wavy curves are due to more frequent evaluation points throughout the day. The simulation is evaluated at a per minute basis whereas the recorded data in Figure 4.3 is collected on a per hour basis. More data points allow the simulation plot to reveal more details of the dynamic nature of power consumption behavior. As to the sudden rise in energy consumption, it is mostly due to the imperfection of the household consumption modeling and simplification of real life scenarios in the simulation. The simulation was configured such that multiple appliances are allowed to start their operations at the same time for all households. Although each appliance needs to roll a dice to get its permission to start, still a bulk of appliances start their operations right away. The household consumption model can be refined to have more precise entries in the appliance consumption library to more closely resemble reality. Nonetheless, the shape of the simulated consumption profile does truly reflect the consumption reality. The times of the minimum and maximum load on the grid match exactly the measured results regardless of the dwelling type shown in Figure 4.3.

After having the shape of the simulated result verified, let us discuss about the accuracy of the simulated values. Unfortunately, the measurements given in [63] (thus in Figure 
4.3) are in terms of $\mathrm{kWh}$-per-square-meter $\left(\mathrm{kWh} / \mathrm{m}^{2}\right)$. The house type and floor area are not included as a factor in the simulation. These factors eventually come down to the unit time consumption of an appliance and the operation duration of such an appliance, and of course the amount of appliances owned by a household. To verify the accuracy of the simulated values, a non-scientific survey was given to a small sample size of people to provide their average daily consumption reported by their local utility company during the months from June to August. The gathered results ranged from $8 \mathrm{kWh} /$ day to $80 \mathrm{kWh} /$ day in hot summer days. The large variation seen in the power consumption among different households is mostly due to the type and number of appliances each home owns. The household with the largest power consumption owns a swimming pool with a pump (and a heat pump) constantly running. This is the major contribution to the household's large power consumption. None of the other surveyed households owns a private pool, and thus neither does the simulation currently contain a model for a swimming pool in the appliance consumption library. Therefore, the $80 \mathrm{kWh} /$ day sample is treated as an outlier from the gathered data set. For all other households, the most frequently appearing average daily consumptions then ranged from $8 \mathrm{kWh} /$ day to $29 \mathrm{kWh} /$ day. The simulated average daily consumption $(23.08 \mathrm{kWh})$ falls well within this range as show in Figure 4.2, and the minimum $(10.01 \mathrm{kWh})$ and maximum $(36.47 \mathrm{kWh})$ simulated daily consumptions are also close to the survey results. Therefore, the simulation results are verified.

\subsection{Base Consumption Profile}

The following figure shows the base consumption vs. traditional consumption profiles. 


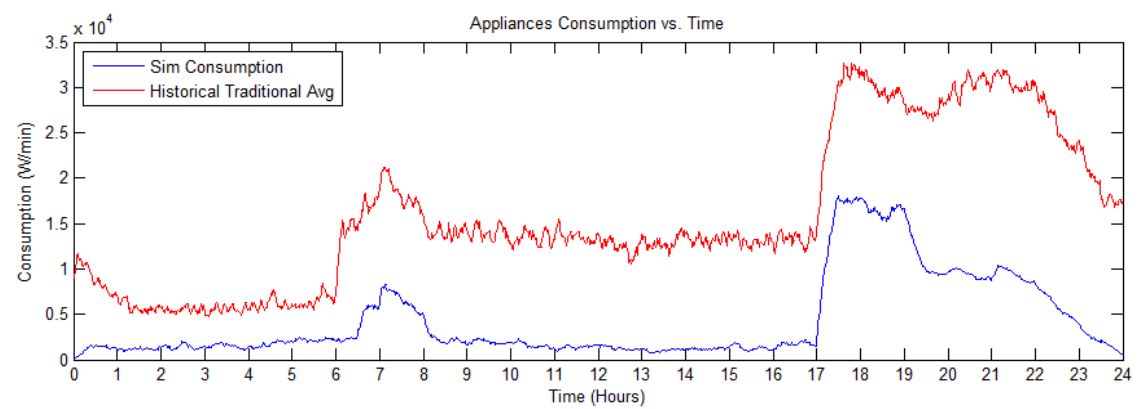

Figure 4.4: Base Consumption vs. Traditional Consumption

As shown in Figure 4.4, the blue line is the base consumption profile of 1000 households, and the red line is the reference traditional consumption profile of 1000 households. Recall that the base consumption profile only captures the consumption behavior of the non-shiftable appliances. These are the appliances which operate as soon as they are needed. Thus coordination logics are not applicable on this type of appliances. On the other hand, the shiftable appliances are subject to coordination, and they are represented by the space between the red line and the blue line. As shown in the figure, the shiftable appliances' consumption represents a large chunk of workable area. This further indicates a large potential to coordination algorithm development. A well designed coordination algorithm may balance the consumption to reduce peak load and/or better utilize energy resources during the low demand period.

\subsection{Traditional Consumption Profile}

Please refer to Figure 4.1 and Figure 4.2 for the illustration of the simulation results of 1000 households under traditional coordination (i.e. no coordination). Looking at the upper subplot of Figure 4.1, what is worth noticing is that the general shape of the consumption profile follows the reference consumption profile closely since both lines 
are generated with no coordination. The peaks and valleys of the consumption profiles happen at the same time with slightly different values. To statistically verify there is no significant difference between two curves, the average household consumptions and their confidence intervals are compared. As seen in Figure 4.2, the confidence interval of the simulated results and the reference values are almost the same $(23.08 \pm 0.34 \mathrm{kWh}$ vs. $23.07 \pm 0.33 \mathrm{kWh}$ ). Observed from Figure 4.1, the two consumption profiles do not overlap completely. The pseudo random data set is able to converge to the same mean. This result statistically confirms that the differences among the two simulations are insignificant. This also aids in demonstrating the stability of the implemented simulation framework.

Since the electricity is traditionally charged with the same price throughout a day, a flat rate of $8 \mathrm{cent} / \mathrm{kWh}$ is used in the simulation. This is shown as the blue line in the lower subplot of Figure 4.1. Under this flat price, the corresponding average household energy costs are calculated to be the same as expected. Both the simulated average cost and the reference average are $1.85 \pm 0.03$ dollars. Traditional electricity usage is not affected by the price of electricity, nor there is any communication or coordination involved. The lower subplot is merely provided for information purpose.

\subsection{Introduction of Electrical Vehicles}

Electrical vehicles are a new type of "household appliance" introduced in recent years. The integration and support of electrical vehicles is also a key driver for smart grid development. It is interesting to see the effect of this power-hungry appliance on the 
power grid. Below is an example of this phenomenon. The simulation was configured such that 1 in 15 households owns an electrical vehicle. Each electrical vehicle is allowed to start charging between $5: 00 \mathrm{pm}$ to $7: 00 \mathrm{pm}$ with the exact time chosen at random. For simplicity, the length of the charging cycle for all electrical vehicles is set to 4.5 hours.
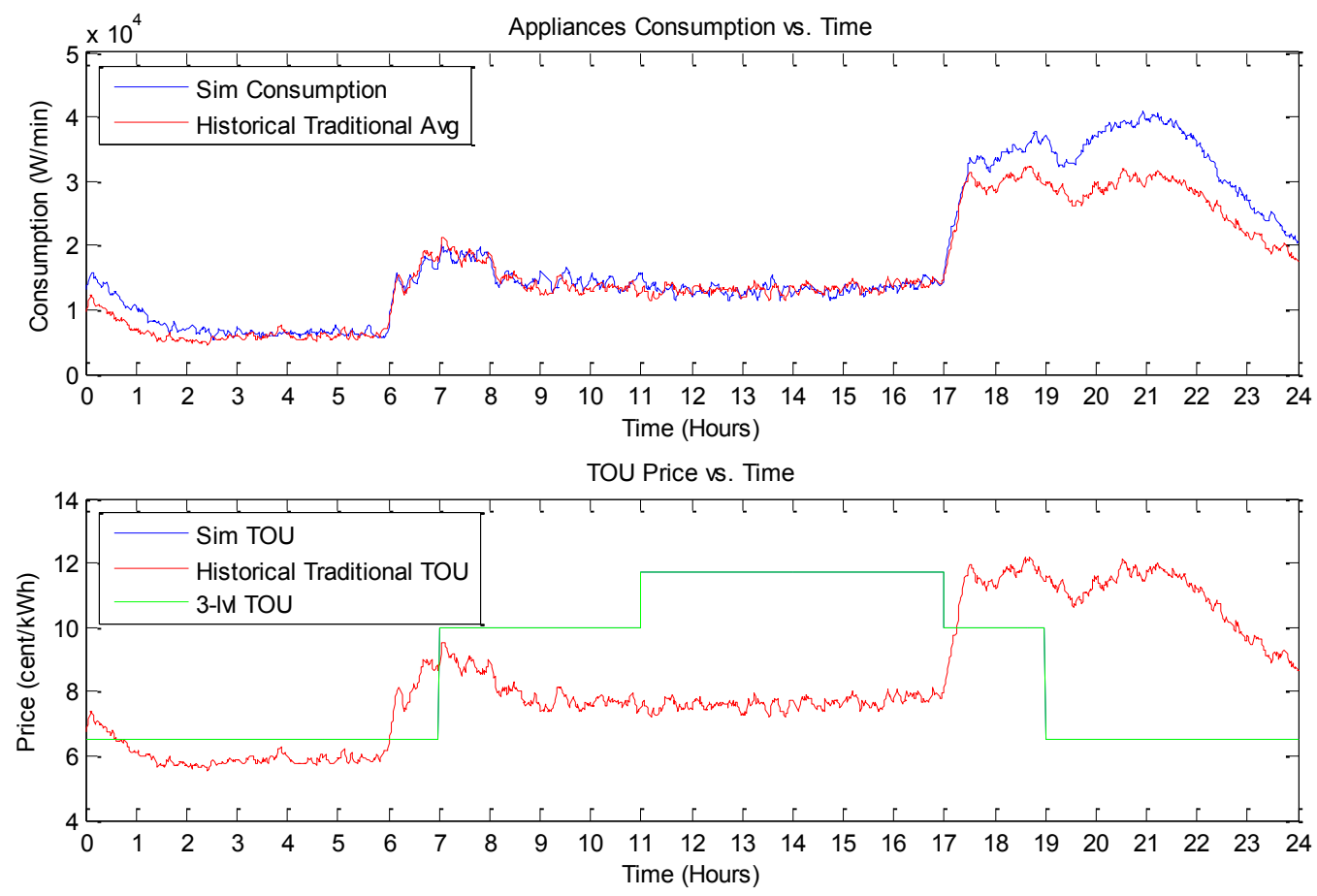

Figure 4.5: Electrical Vehicle Effect Plot

As shown in Figure 4.5 and Figure 4.6, assuming electrical vehicle owners start the charging cycle as soon as they return home from work between 5:00pm and 7:00pm, 67 $\left(1 / 15^{*} 1000 \sim 67\right)$ electrical vehicles draw a significant amount of electricity off the power grid. This can be verified by comparing the confidence intervals of the average consumption from the simulation and from the reference profile. The two intervals are mutually exclusive, which indicates that the differences between the two consumption curves are significant. The difference is also visible from Figure 4.5 as shown by the 
block of additional electricity being consumption from around 5:30pm to midnight. The peak consumption rises from $1940.09 \mathrm{kWh}$ to $2451.30 \mathrm{kWh}$ when compared to the reference consumption profile. The highest household consumption reaches all the way to $78.89 \mathrm{kWh} /$ day, where the traditional highest value is around $35 \mathrm{kWh} /$ day, and the highest household electricity cost reaches $\$ 6.17$, compared to a cost of around $\$ 3.50$ in the absence of an electrical vehicle. To conclude, electrical vehicles do have a big impact on the power grid. Both the residential consumer and the power grid in general would benefit from smart grid load balancing studies to minimize the impact of the introduction of electrical vehicles. The generated consumption profile (blue line) will be used as reference in this thesis when electrical vehicles are included in the simulation.

\begin{tabular}{|c|c|}
\hline Number of households simulated: & 1000 \\
\hline Coordination method used: & Traditional Consumption Simulation \\
\hline TOU price scheme used: & OnPeak-MidPeak-OffPeak Pricing \\
\hline Average household daily consumption: & $26.04 \pm 0.79 \mathrm{kWh}$ \\
\hline Reference average daily consumption: & $23.07 \pm 0.33 \mathrm{kWh}$ \\
\hline Lowest household daily consumption: & $9.58 \mathrm{kWh}$ \\
\hline Highest household daily consumption: & $78.89 \mathrm{kWh}$ \\
\hline Average household daily cost: & $2.21 \pm 0.06$ dollars \\
\hline Reference average daily cost: & $1.85 \pm 0.03$ dollars \\
\hline Lowest household daily cost: & 0.82 dollars \\
\hline Highest household daily cost: & 6.17 dollars \\
\hline Peak daily total consumption: & $2451.30 \mathrm{~kW} / \mathrm{h}$ occurred at $21: 56$ \\
\hline Reference peak consumption: & $1940.09 \mathrm{~kW} / \mathrm{h}$ occurred at $19: 41$ \\
\hline Total consumption statistics: & Mean: $1085.00 ; \quad$ Standard Deviation: 630.71 \\
\hline Reference consumption statistics: & Mean: 961.43; $\quad$ Standard Deviation: 494.4343 \\
\hline
\end{tabular}

Figure 4.6: Electrical Vehicle Effect Report 


\section{Chapter 5: Coordination and Pricing Schemes}

This chapter presents the simulation results of the implemented coordination models and pricing schemes. The results will be compared with the ones presented and validated in Chapter 4.

\subsection{Programmable Appliances Consumption Profile}

Recall that programmable appliances can schedule their operations based on time. A well known example of this type of appliances is the programmable thermostat. User sets a temperature profile based on time across a day, and the heating or cooling appliances operate to meet the temperature profile. This section demonstrates the impact of this type of appliance on the power grid. Since the accuracy of the simulation framework has been demonstrated in Section 4.5, simulations in this section will only employ 100 households instead of 1000 .

\subsubsection{Basic Programmable Appliances Operation}

Let us simplify the model and assume that we can schedule a single appliance's operation profile. Below is an example of scheduling the air conditioner to turn off after occupants left home for work around 9:00am, and to turn back on around 4:00pm when they return home. 

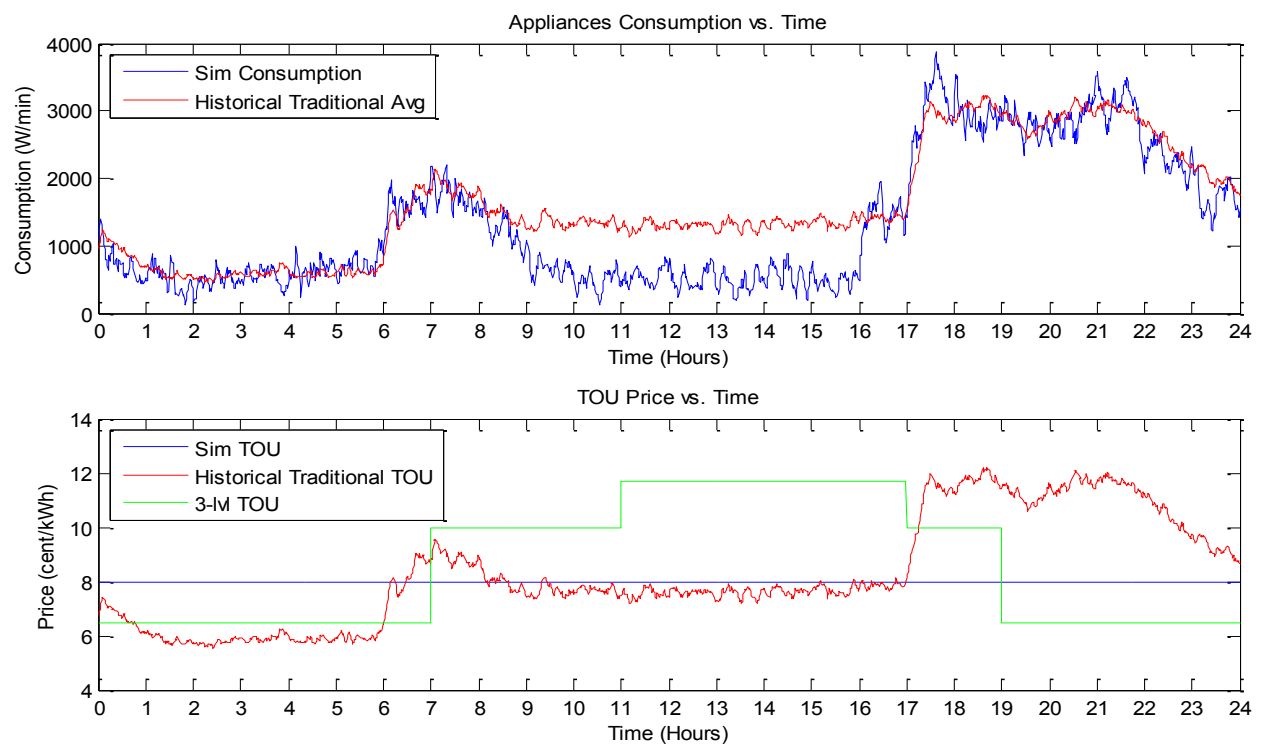

Figure 5.1: Programmable Appliance Consumption Profile

$==\mathrm{E}=\mathrm{=}=\mathrm{=}=\mathrm{=}=\mathrm{=}=\mathrm{=}=\mathrm{=}=\mathrm{=}=$ Simulation Report

Number of households simulated:

100

Coordination method used:

Programmable Appliances Simulation

TOU price scheme used:

Flat Rate Pricing

Average household daily consumption:

$19.20 \pm 1.07 \mathrm{kWh}$

Reference average daily consumption:

$23.07 \pm 0.33 \mathrm{kWh}$

Lowest household daily consumption:

$10.50 \mathrm{kWh}$

Highest household daily consumption:

$33.02 \mathrm{kWh}$

Average household daily cost:

$1.54 \pm 0.09$ dollars

Reference average daily cost

$1.85 \pm 0.03$ dollars

Lowest household daily cost:

0.84 dollars

Highest household daily cost:

2.64 dollars

Peak daily total consumption:

$232.66 \mathrm{~kW} / \mathrm{h}$ occurred at $18: 37$

Reference peak consumption:

$194.01 \mathrm{~kW} / \mathrm{h}$ occurred at 19:41

Total consumption statistics:

Mean: 80.02; Standard Deviation: 58.85

Reference consumption statistics:

Mean: 96.14; Standard Deviation: 49.44.09 
As shown in the upper subplot of Figure 5.1, compared to the red reference line, the consumption profile of the current simulation displays an obvious drop between 8:00am and 4:00pm. Also, a step-up of consumption appears at around 4:00pm due to the precooling period before the occupants return home. This particular simulation also reports that the peak consumption $(232.66 \mathrm{kWh})$ is higher than the reference one $(194.01 \mathrm{kWh})$. However, the average household daily consumption $(19.20 \mathrm{kWh})$ is lower than the reference usage $(23.07 \mathrm{kWh})$. All this indicates a significant change in the consumption. To statistically verify the significant of this change, the confidence intervals are again employed for the comparison. As shown Figure 5.2, the confidence interval for the average energy consumption under basic programmable appliances is mutually exclusive with the reference one. This indicates that the changes are statistically significant. To see how much of a change in the consumption results from having basic programmable appliances, the following equation calculates how much savings the simulation actually achieves.

Equation 5.1:

$$
\%_{\text {consumption-saving }}=\frac{\mathrm{C}_{\text {Ref-consumption }}-\mathrm{C}_{\text {consumption }}}{\mathrm{C}_{\text {Ref-consumption }}} * 100 \%
$$

In Equation 5.1, $\mathrm{C}_{\text {consumption }}$ is the cost of the daily consumption from the current simulation, and $\mathrm{C}_{\text {Ref-consumption }}$ is the cost of the daily consumption of the reference consumption profile. Using this equation, the percentage saving of turning off the air conditioner during the day time is about $17 \%$.

The simulation uses a flat rate of 8 cent $/ \mathrm{kWh}$ for pricing as shown by the blue horizontal line. With this same price, the reported averaged daily cost is $\$ 1.54$, which is lower than 
the referencing average of $\$ 1.85$. The percentage saving in terms of money is defined with the following equation:

Equation 5.2:

$$
\%_{\text {money-saving }}=\frac{\mathrm{S}_{\mathrm{Ref}-\text { consumption }}-\mathrm{S}_{\text {consumption }}}{\mathrm{S}_{\text {Ref-consumption }}} * 100 \%
$$

In this equation " $S$ " denotes the spending or daily cost of the consumption. Using this equation, the monetary percentage saving of turning off the air conditioner during day time is about $17 \%$, the same as the consumption percentage saving. This is due to the $1: 1$ ratio between consumption saving to monetary saving under the flat rate pricing scheme.

Overall, the programmable air conditioner (or a thermostat) does seem to lower the household total consumption. Better or smarter algorithms, such as in [31], can optimize the scheduling of programmable appliances to better reduce energy consumption. Residential consumers are able to obtain some savings on the electricity cost on a daily basis. If the schedule fits the occupancy status well, savings are a function of time that an expensive appliance can be turned off.

\subsubsection{Effect of Different Pricing Schemes}

This section demonstrates the effect of two different pricing schemes on the programmable appliances' consumption profile. The first scheme, called OnPeakMidPeak-OffPeak, is similar to the 3 level pricing structured used in Ottawa. For the second scheme, called linear scale, the cost of electricity is derived from the real-time consumption. 


\subsubsection{OnPeak-MidPeak-OffPeak Pricing Scheme}

The first scheme is the OnPeak-MidPeak-OffPeak pricing scheme. As the name suggests, the daily price profile is divided into three different pricing periods. The shape and value of this price profile is usually static and set by utility companies. Please see the following figures for an illustration of such pricing scheme and the effect of air conditioner scheduling under this pricing scheme.
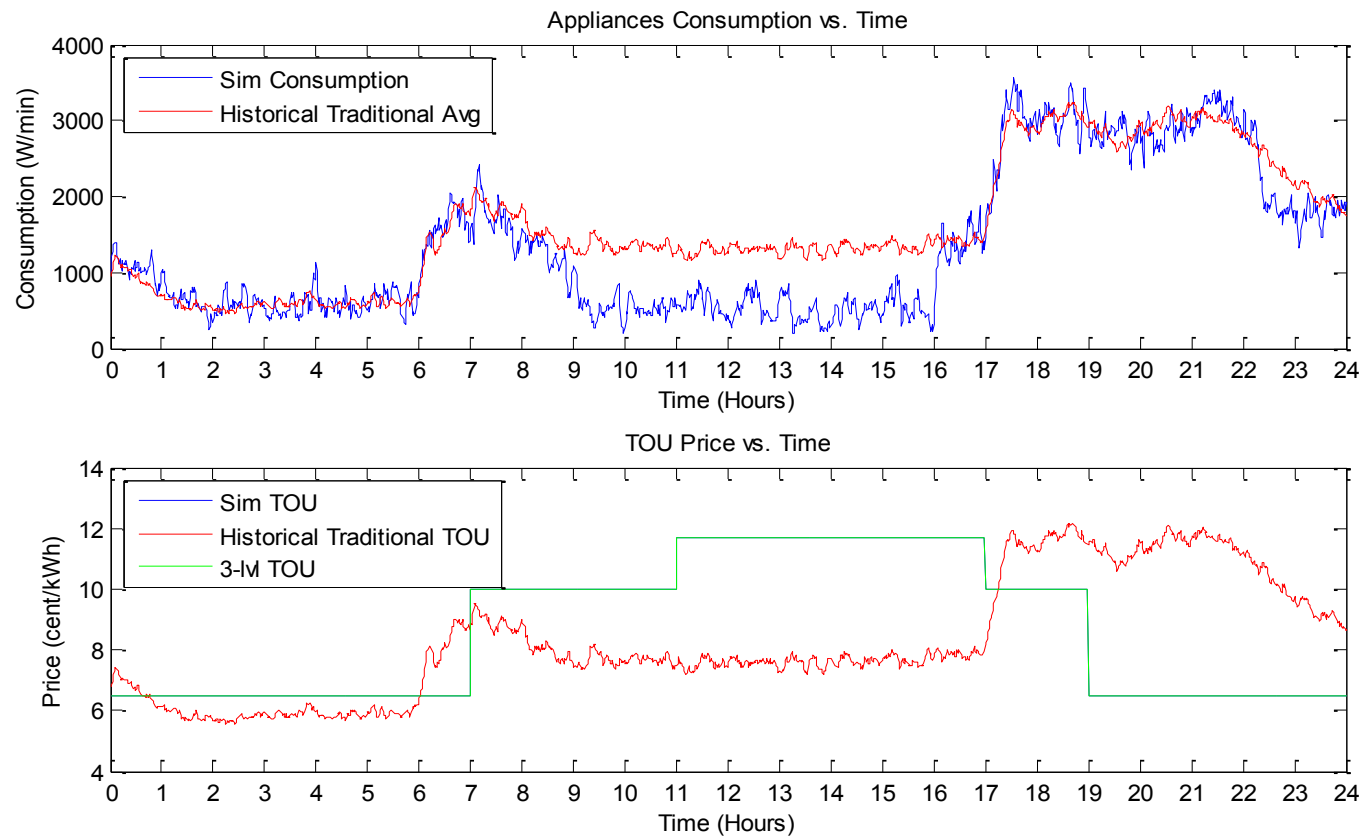

Figure 5.3: Programmable Appliance Consumption Profile with OnPeak-MidPeak-OffPeak Pricing

As shown in Figure 5.3, the generated consumption profile largely resembles the one shown in Figure 5.1. An obvious drop in consumption is presented between 9:00 and 16:00. Minor variations in shape come from the randomization mechanism. 


\begin{tabular}{|c|c|}
\hline Number of households simulated: & 100 \\
\hline Coordination method used: & Programmable Appliances Simulation \\
\hline TOU price scheme used: & OnPeak-MidPeak-OffPeak Pricing \\
\hline Average household daily consumption: & $19.39 \pm 0.97 \mathrm{kWh}$ \\
\hline Reference average daily consumption: & $23.07 \pm 0.33 \mathrm{kWh}$ \\
\hline Lowest household daily consumption: & $11.20 \mathrm{kWh}$ \\
\hline Highest household daily consumption: & $29.40 \mathrm{kWh}$ \\
\hline Average household daily cost: & $1.59 \pm 0.08$ dollars \\
\hline Reference average daily cost: & $2.00 \pm 0.05$ dollars \\
\hline Lowest household daily cost: & 0.92 dollars \\
\hline Highest household daily cost: & 2.40 dollars \\
\hline Peak daily total consumption: & $213.84 \mathrm{~kW} / \mathrm{h}$ occurred at $18: 33$ \\
\hline Reference peak consumption: & $194.01 \mathrm{~kW} / \mathrm{h}$ occurred at $19: 41$ \\
\hline Total consumption statistics: & Mean: $80.79 ; \quad$ Standard Deviation: 58.23 \\
\hline Reference consumption statistics: & Mean: 96.14; $\quad$ Standard Deviation: 49.44 \\
\hline
\end{tabular}

Figure 5.4: Programmable Appliance Consumption Report with OnPeak-MidPeak-OffPeak Pricing

To verify there is no significant change from Figure 5.1, the confidence intervals are compared against Figure 5.4. For Figure 5.1, the average household consumption has a confidence interval of $19.20 \pm 1.07 \mathrm{kWh}$, whereas the current simulation yields an average consumption of $19.39 \pm 0.97 \mathrm{kWh}$. The confidence intervals from the two simulations are overlapping and the average value of each falls into the confidence interval of the other. This indicates the two consumption profiles have no significant difference in terms of daily consumption on average. Plus the general shapes of the consumption profiles are similar, this provides a good basis for comparison. According to Equation 5.1, there is about $16 \%$ of saving on the consumption. It is again very close to 
the result obtained with the flat rate pricing case. On the pricing side, since the simulation uses the OnPeak-MidPeak-OffPeak pricing scheme, the referencing green line and the blue line overlap in the lower subplot of Figure 5.3. With this same pricing scheme, the reported average daily cost is $\$ 1.59$, which is lower than the referencing traditional average of $\$ 2.00$. The difference is again shown to be statistically significant by the confidence intervals. With equation 5.2, the calculated monetary percentage saving is $20 \%$.

Comparing these results to the previous simulation with the flat rate pricing scheme, the percentage saving on the consumption $(16 \%)$ is slightly lower than the flat rate pricing case $(17 \%)$. This is due to the randomization from two different simulations. What is more interesting is the relationship to the monetary percentage saving value. With the flat rate pricing simulation, the monetary percentage saving is $17 \%$ which is the same as the consumption percentage saving as explained before. On the other side, with the OnPeakMidPeak-OffPeak pricing, the monetary percentage saving is $20 \%$ which is higher than its consumption percentage saving counterpart. This result is expected because the scheduling purposely avoids air condition operation during the high price period. Since the price per saved consumption is more valuable than the flat rate case in terms of money, the monetary percentage saving is thus higher. So far, the operations scheduling with OnPeak-MidPeak-OffPeak pricing scheme seems to be a good candidate for consumers to use in smart grid. However, due to the previously turned off air conditioners, the room temperature of households are very high. It takes much more effort to cool the house down before the arrival of the occupants. It is modeled in the 
simulator that air conditioners have higher chance to start operate around $4: 30 \mathrm{pm}$ to 6:00pm and they operate longer for this period to simulate this situation. As a result, the peak consumption rises from $194.01 \mathrm{kWh}$ to $213.84 \mathrm{kWh}$ around $6: 00 \mathrm{pm}$. This is not a desired consequence of the overall smart grid design. A rise in peak consumption may cause the need for building more generators or power plants, which is the opposite of the goal of smart grid development. Therefore, a better coordination method is required.

\subsubsection{Linear scale pricing scheme}

The next simulation demonstrates the effect of the linear scale pricing scheme on programmable air conditioners. Recall that the linear pricing is a one-to-one scale matching between consumption and the time of use price.
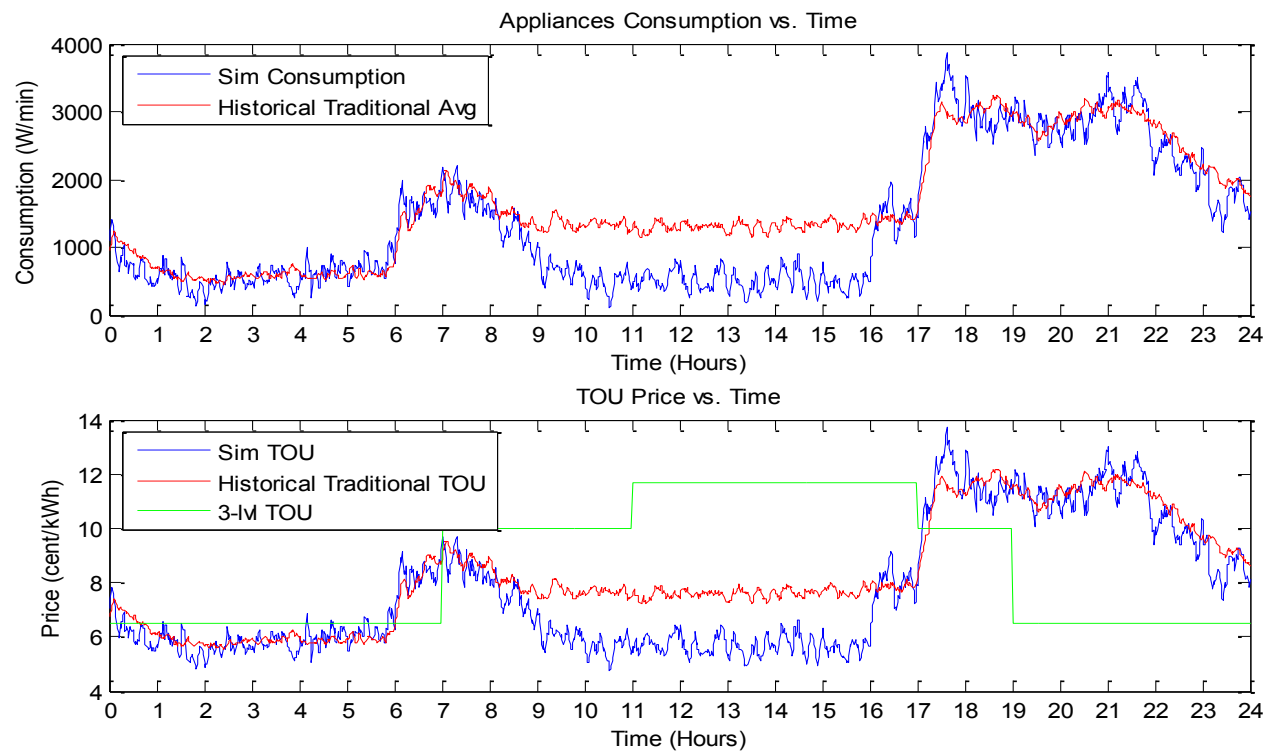

Figure 5.5: Programmable Appliance Consumption Profile with Linear Scale Pricing

As shown in Figure 5.5, the overall shape of the consumption profile resembles the ones from the previous two simulations. What differs is the linear price profile in the lower 
subplot. As shown, the shape of this line is identical to the consumption line in the upper subplot. The scale of this line is set such that its valley is lower than the off-peak value of the OnPeak-MidPeak-OffPeak pricing scheme; and its peak is higher than the on-peak value. The reason for doing so is to further encourage consumption at off-peak hours, and to tighten usage in on-peak hours. The scale of this line is easily modifiable, which is convenient for price regulation used by utility companies for example.

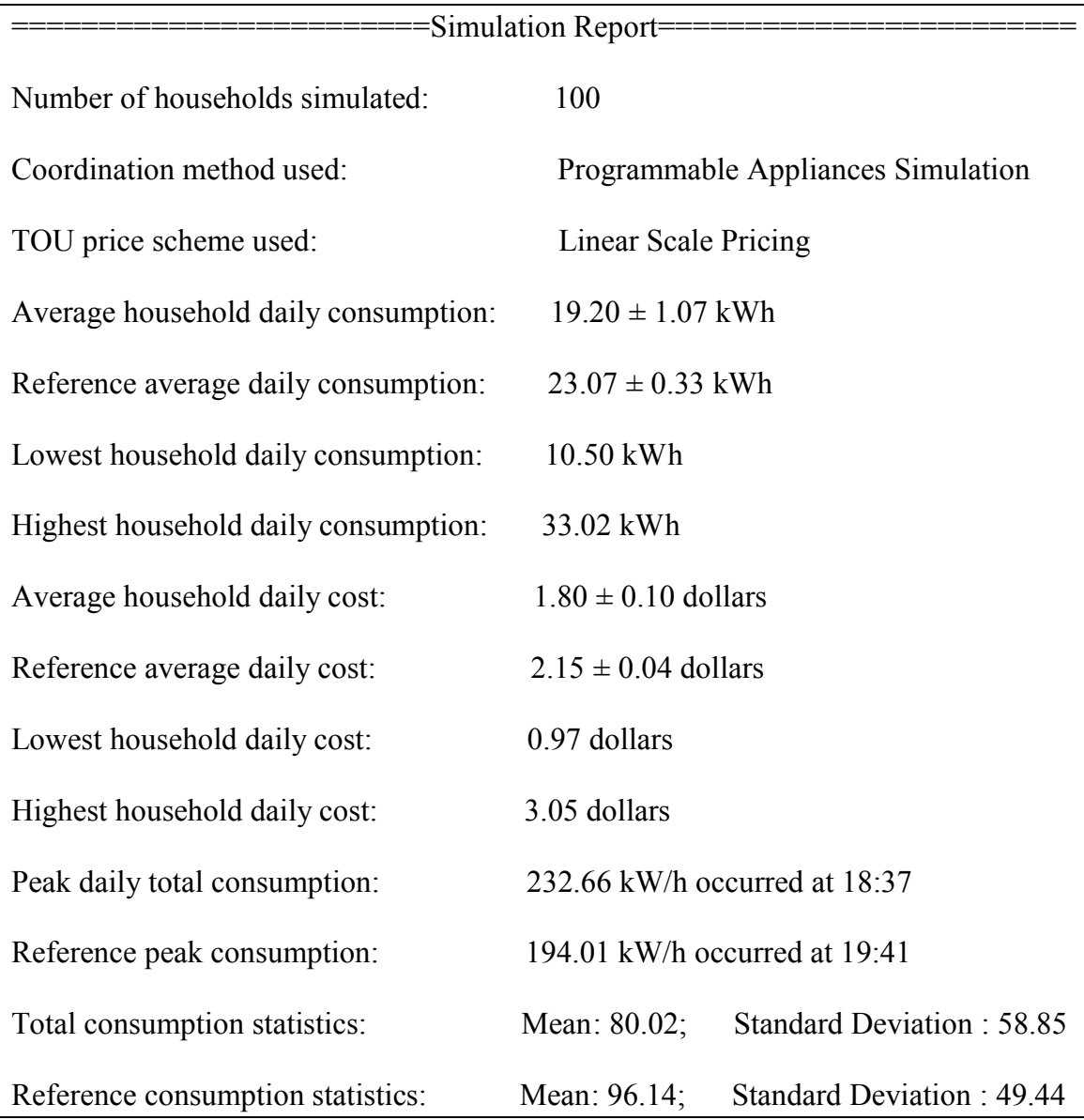

Figure 5.6: Programmable Appliance Consumption Report with Linear Scale Pricing

Under this linear pricing scheme, the calculated consumption percentage saving and monetary percentage saving are $17 \%$ and $16 \%$ respectively. Surprisingly, the monetary saving ratio is actually slightly lower, which means a consumer achieves a little less 
saving off their reduction on energy consumption. This unfortunate result is again due to the difference in the value of energy in different scenarios. After scheduling the air conditioners to turn off during the day, the value of energy drops a little under the linear price scheme because there is less demand for energy. In other words, the value of the energy depreciates when reducing the energy demand. In the end, the saved energy is not worth as much as before.

The above results may sound disappointing. However, keep in mind that the simulation only includes residential energy usage; commercial usage and all other usage are excluded. It is well-known that commercial peak usage happens during the daytime between 9:00am and 5:00pm. Therefore, the actual time of use price during this time period is very high. Regardless whether the value of energy depreciates after saving, it is a large amount of money saved if not operating in this time period. Also, no realistic supply-side model has been included in the simulation framework yet. Utility companies can turn off a number of generators during low-demand period. As a consequence of this change, the value of energy actually increases because there is less available energy being supplied. A complete supply-demand model is highly desirable to reveal the true value of energy. Here, in this study, we are only able to characterize and demonstrate the effects of different demand-side approaches, and try to find a midpoint of load balancing and residential consumption savings.

Overall, the linear pricing scheme is able to faithfully reveal the value of energy and should be used as a guideline of smart grid coordination algorithm development and load 
balancing research. As illustrated in Figure 5.5, although there are energy savings due to scheduling, the higher peak consumption due to the extra cooling effort from air conditioners is still present. As shown in the simulation report, the standard deviation after scheduling (58.85) is higher than reference usage (49.44). This means a greater dispersion resulted on the power grid. This effect is the opposite of the load balancing goal, and thus needs to be improved upon. What is lacking is an effective response system or coordination algorithm to utilize the dynamic pricing scheme. An improved solution could create a great benefit for society.

\subsubsection{Effect of Electrical Vehicle Charging Scheduling}

Section 5.1.2 demonstrated that operation scheduling with linear pricing resulted in a lower monetary rate of return from the reduction in the usage of electricity. There is another type of scheduling that does not reduce energy usage but is still able to achieve a positive rate of return by shifting an appliance's operation time overnight. A typical example of this type of appliance is the electrical vehicle. The following figures demonstrate the effect of scheduling this power-hungry appliance to move its charging cycle overnight when energy demand/consumption is at its minimum.

The first result demonstrates the effect of scheduling the charging operation under the OnPeak-MidPeak-OffPeak pricing scheme. The electrical vehicles are scheduled to start their charging cycle around midnight instead of in the evening. The charging period is still 4.5 hours for simplicity. The following figures are the results of this setting. 

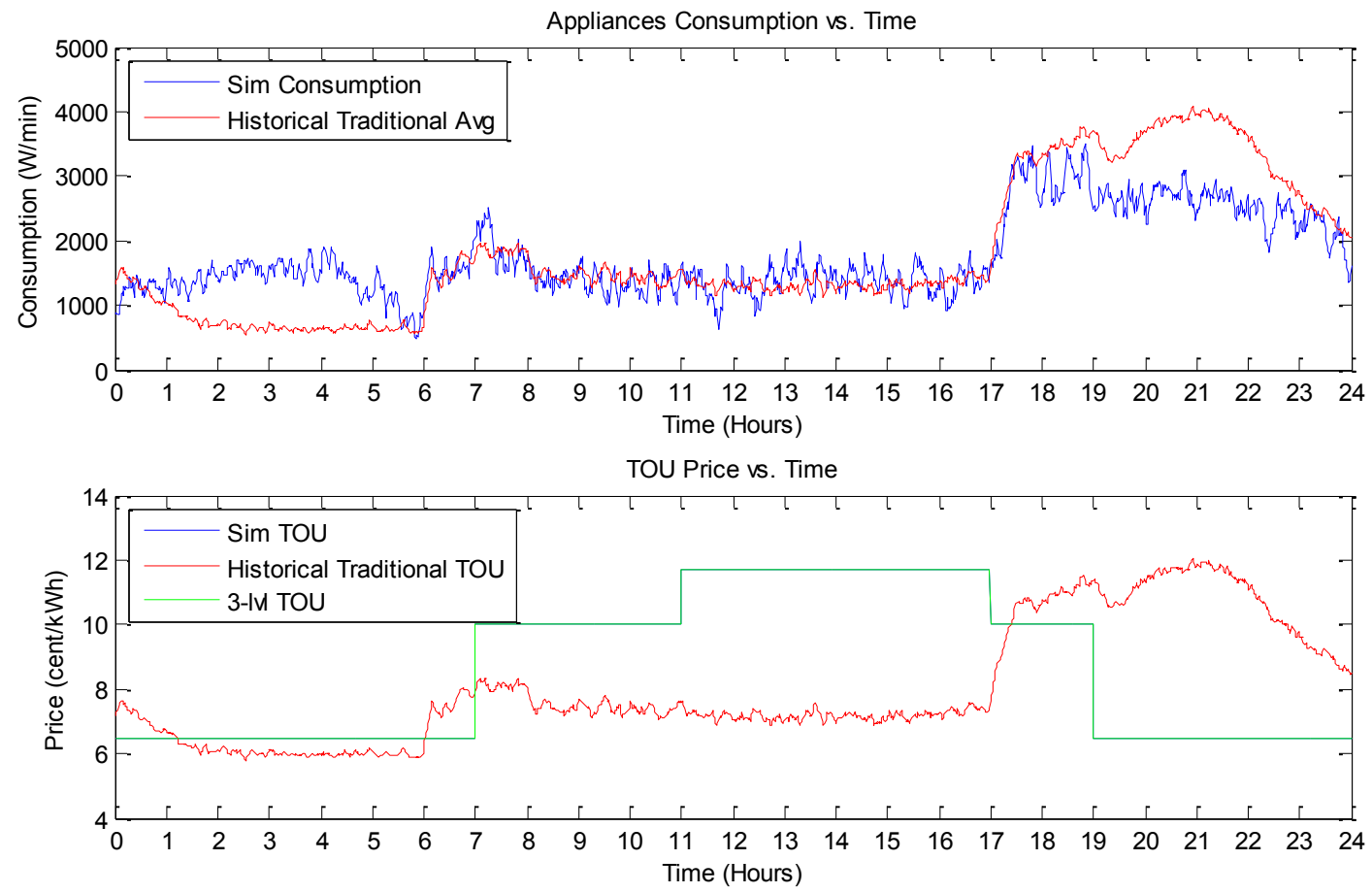

Figure 5.7: EV Charging Scheduling Under OnPeak-MidPeak-OffPeak Pricing Sceme

As shown in Figure 5.7, the red reference line is profiled under traditional usage plus the introduction of electrical vehicles that was illustrated in Figure 4.5 of Section 4.6. With this reference line, it is very obvious to see the effect of shifting the electrical vehicle charging operation. The bulk of consumption, which used to occur during the evening, is delayed to happen overnight and into the next day. To see if there is a significant change after the shifting, we analyze the consumption confidence intervals. As we can see, the confidence interval of the reference average falls entirely within the confidence interval of the simulated curve. This is mainly due to the difference in the sample size of the current simulation versus the reference one; also the introduction of electrical vehicles enlarges the confidence interval as there is more variance in households' consumptions. As a result of the operation shifting, the calculated consumption percentage saving and 
the monetary percentage saving are both around $3 \%$. The statistically insignificant change in consumption also results in insignificant change in the energy cost. This is because the value of the shifted consumptions remains the same during the operation. As shown in Figure 5.7, the block of consumption moved from the evening to the overnight is always charged based on the off-peak price.

\begin{tabular}{|c|c|}
\hline Number of households simulated: & 100 \\
\hline Coordination method used: & Programmable Appliances Simulation \\
\hline TOU price scheme used: & OnPeak-MidPeak-OffPeak Pricing \\
\hline Average household daily consumption: & $25.22 \pm 2.59 \mathrm{kWh}$ \\
\hline Reference average daily consumption: & $26.04 \pm 0.79 \mathrm{kWh}$ \\
\hline Lowest household daily consumption: & $9.82 \mathrm{kWh}$ \\
\hline Highest household daily consumption: & $79.24 \mathrm{kWh}$ \\
\hline Average household daily cost: & $2.14 \pm 0.19$ dollars \\
\hline Reference average daily cost: & $2.21 \pm 0.06$ dollars \\
\hline Lowest household daily cost: & 0.88 dollars \\
\hline Highest household daily cost: & 5.94 dollars \\
\hline Peak daily total consumption: & $210.20 \mathrm{~kW} / \mathrm{h}$ occurred at 19:50 \\
\hline Reference peak consumption: & $245.13 \mathrm{~kW} / \mathrm{h}$ occurred at $21: 56$ \\
\hline Total consumption statistics: & Mean: 105.06; $\quad$ Standard Deviation: 37.42 \\
\hline Reference consumption statistics: & Standard Deviation: 63.07 \\
\hline
\end{tabular}

\section{Figure 5.8: EV Charging Scheduling Under OnPeak-MidPeak-OffPeak Report}

There is no real monetary benefit to individual households. However, the real benefit of the operation shifting under the OnPeak-MidPeak-OffPeak pricing scheme comes in a reduction of peak consumption. The peak consumption drops from $245.13 \mathrm{kWh}$ to $210.20 \mathrm{kWh}$; and the standard deviation of the curve also drops from 63.07 to 37.42 . All 
these results mean that the power drawn off the power grid is more steady and constant. The generated energy is utilized evenly, and power generators can reduce the starting up/stopping of power plants, increasing their lifetime. A drop in the peak consumption can potentially eliminate the need to build more power plants to cover the ever growing demand for electricity. Overall, operation scheduling for electrical vehicles is a promising solution.

However, since the majority of the consumption from charging electrical vehicles with or without shifting happens during Off-Peak hours already (after 7:00pm), there is no significant monetary benefit to individual household consumers. To enable this incentive, the next simulation demonstrates the same electrical vehicle scheduling under the linear pricing scheme.
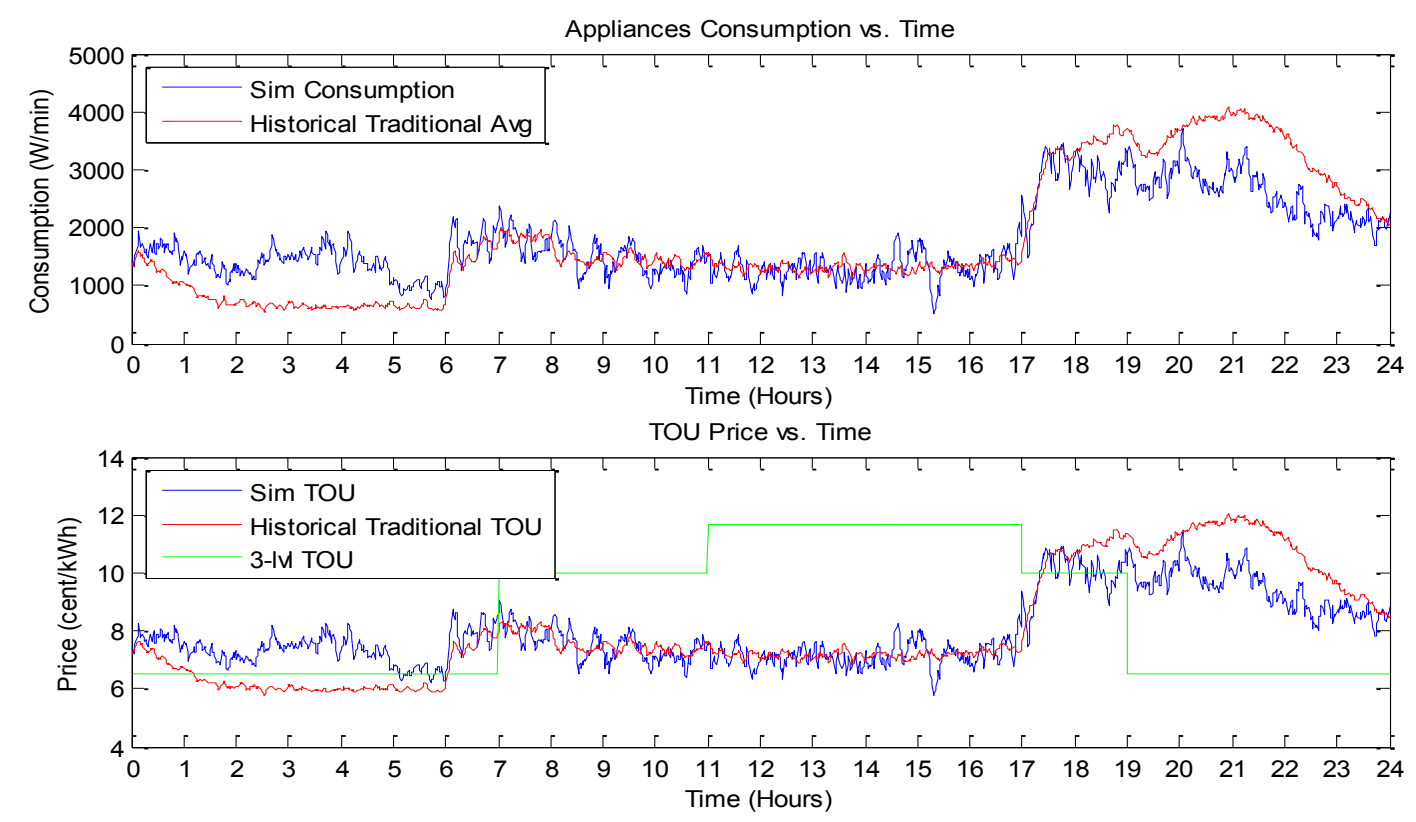

Figure 5.9: Charging Scheduling Under Linear Pricing Sceme 


\begin{tabular}{|c|c|}
\hline Number of households simulated: & 100 \\
\hline Coordination method used: & Programmable Appliances Simulation \\
\hline TOU price scheme used: & Linear Scale Pricing \\
\hline Average household daily consumption: & $25.61 \pm 2.25 \mathrm{kWh}$ \\
\hline Reference average daily consumption: & $26.04 \pm 0.79 \mathrm{kWh}$ \\
\hline Lowest household daily consumption: & $12.35 \mathrm{kWh}$ \\
\hline Highest household daily consumption: & $77.19 \mathrm{kWh}$ \\
\hline Average household daily cost: & $2.16 \pm 0.13$ dollars \\
\hline Reference average daily cost: & $2.37 \pm 0.07$ dollars \\
\hline Lowest household daily cost: & 1.13 dollars \\
\hline Highest household daily cost: & 6.05 dollars \\
\hline Peak daily total consumption: & $223.10 \mathrm{~kW} / \mathrm{h}$ occurred at $20: 3$ \\
\hline Reference peak consumption: & $245.13 \mathrm{~kW} / \mathrm{h}$ occurred at $21: 56$ \\
\hline Total consumption statistics: & Mean: $106.73 ; \quad$ Standard Deviation: 39.74 \\
\hline Reference consumption statistics: & Mean: 108.50; $\quad$ Standard Deviation: 63.07 \\
\hline
\end{tabular}

\section{Figure 5.10: Charging Scheduling Under Linear Pricing Scheme}

As shown in Figure 5.9, the effect of delaying the electrical vehicle charging cycle to overnight is the same as depicted in Figure 5.7. Again, the consumption confidence intervals indicate that there is no statistically significant difference in the change. The calculated consumption percentage saving is about $2 \%$ due to randomization. However, what has made a big change is the corresponding monetary percentage saving, which is calculated to be about $9 \%$. This change is shown to be statistically significant by the confidence intervals. The $95 \%$ confidence interval of the average household daily cost is mutually exclusive from its reference. Why would electrical vehicle charging operation 
scheduling be able to always achieve a positive rate of return under the linear pricing scheme? The following figure explains the reason.
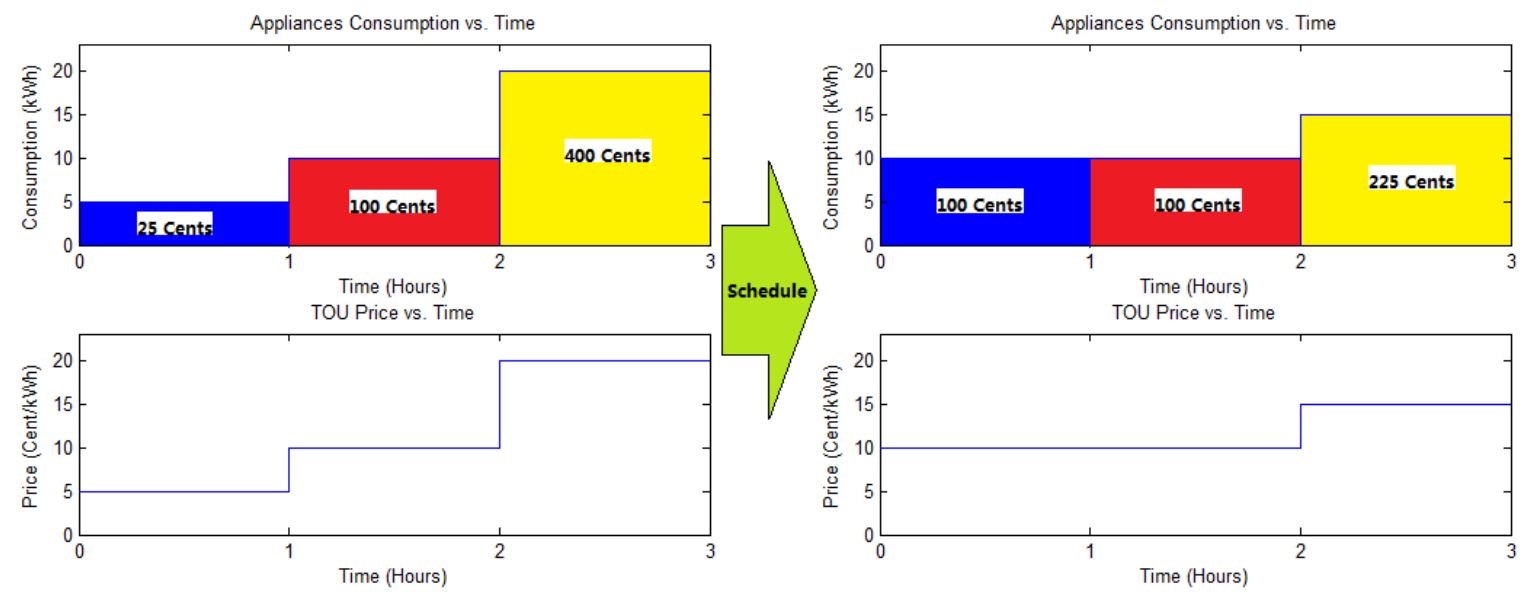

Figure 5.11: EV Charging Scheduling Explaination

To simplify the problem, let us assume there are only three hours in a day. The left plot in Figure 5.11 shows the household daily consumption and the TOU prices of the day before performing electrical vehicle charging operation scheduling; and the plot to the right is the effect after scheduling. In the left plot, the three hours of the day consume $5 \mathrm{kWh}, 10 \mathrm{kWh}$, and $20 \mathrm{kWh}$ of energy respectively; and its linear time of use price is 5 cents per $\mathrm{kWh}$ for the first hour, 10 cents per $\mathrm{kWh}$ for the second hour, and 20 cents per $\mathrm{kWh}$ for the third hour. To calculate the cost of consuming energy in the first hour we have $5 \mathrm{kWh} * 1 \mathrm{~h} * 5 \mathrm{cent} / \mathrm{kWh}=25$ cents for that hour. Therefore, the daily cost on energy for the left plot is $25+100+400=525$ cents. In the plot to the right, what the scheduling does is that it merely moved $5 \mathrm{kWh}$ worth of consumption from the third hour to the first hour. The total consumption of the day remains the same. However, with this change, the total daily cost of consumption becomes $100+100+225=425$ cents. There is a 100 cents 
saving just by shifting the operation. Therefore consumers are able to enjoy the same amount of energy at lower cost.

This is a great incentive to get consumers to be involved in smart grid projects. However, this may not be a scalable solution due to the fact that the scheduling method is not dynamic. In this example, only the electrical vehicles are included in the simulation. There are other appliances that can undergo the same scheduling scheme, such as dishwashers and washing machines. If this manual scheduling operation is overdone, the result may raise the time of use price in the first hour higher than in the third hour, and thus, results in a negative rate of return. Therefore, a more controllable and dynamic method is desired in coordinating appliances' operations. The next section illustrates one simple dynamic solution.

\subsection{Threshold-Controlled Appliances Consumption Profile}

Threshold-controlled appliances make their decision about whether to start operation based on the price of electricity. A threshold price is predetermined by the user or the utility company, and the appliance will only start operation once the electricity price is lower than that threshold. It is very obvious that this coordination method does not work well with static pricing schemes including the OnPeak-MidPeak-OffPeak pricing scheme. This is because the time of use prices are known ahead of time. Operation scheduling based on that known price scheme is enough, but it is not able to regulate the consumption. Therefore, only dynamic pricing schemes, such as the linear pricing scheme, are truly feasible with threshold-controlled operations. The figure below 
illustrates the effect of setting thresholds on electrical vehicles, dishwashers and washing machines to only start their operation when the electricity price is lower than 9 cent $/ \mathrm{kWh}$. For simplicity, all the controlled appliances in all households follow the same threshold.
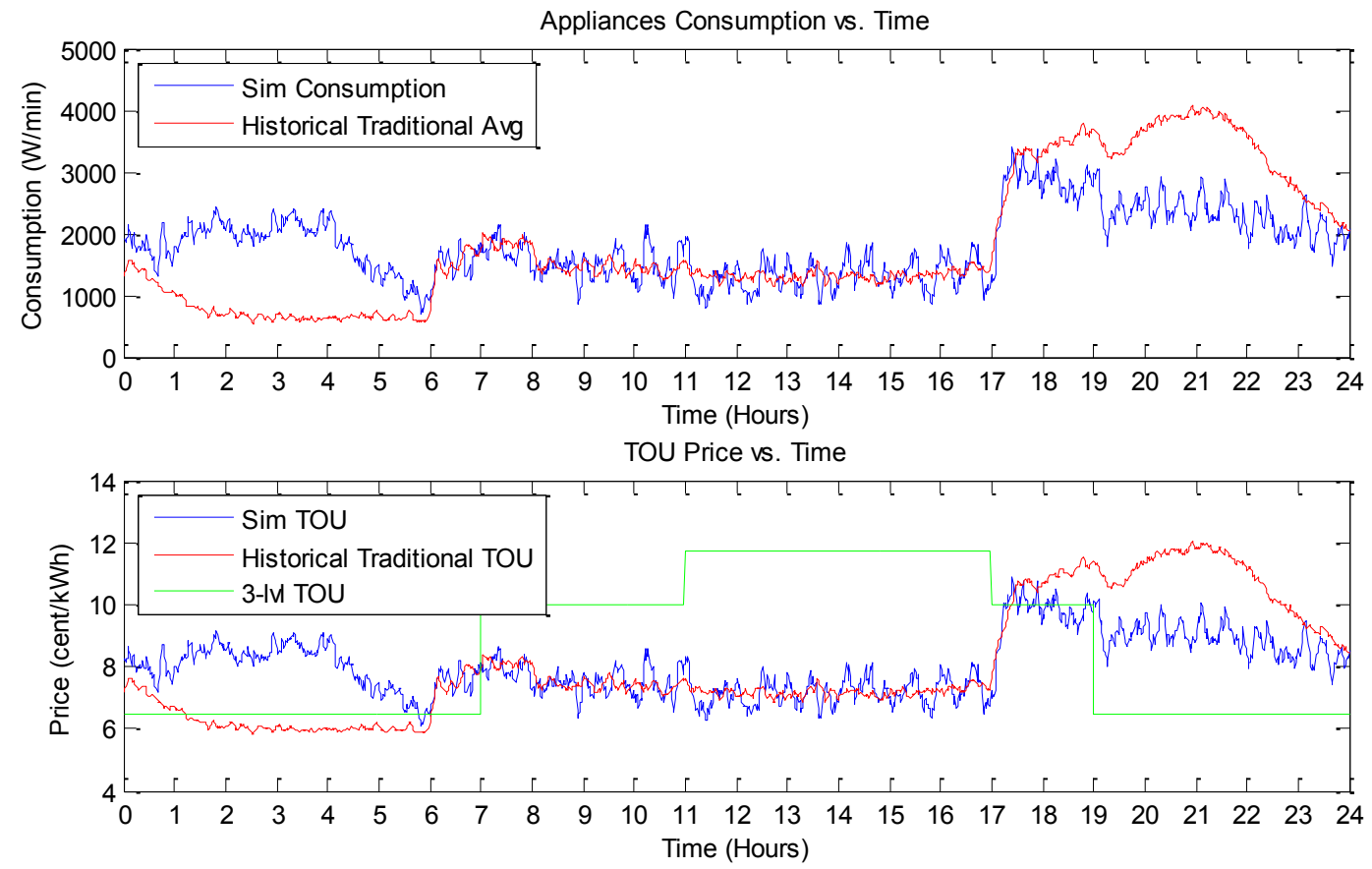

Figure 5.12: Threshold Controlled Appliance Consumption Plots

As shown in Figure 5.12, the consumption after 5:00pm is a more even line which is governed by the price threshold set to 9 cent $/ \mathrm{kWh}$. The controlled appliances need to check the time of use price when trying to start their operation. The previously started appliances contribute to raising the electricity price under the linear pricing scheme. Once the price is higher than the preset threshold, the entry of new appliances is prevented. These appliances keep listening to the time of use price announcements from the smart grid and then retry when a lower price is received. For simplicity, these waiting appliances will need to roll their dice again when a low price is received. There is no 
ticket assigned to them based on order of queuing, nor is there any type of contention algorithm implemented in the current model. Future work can focus on this area. The current study only focuses on exploring the impacts of a simple coordination and the study of metro-scale grid behavior.

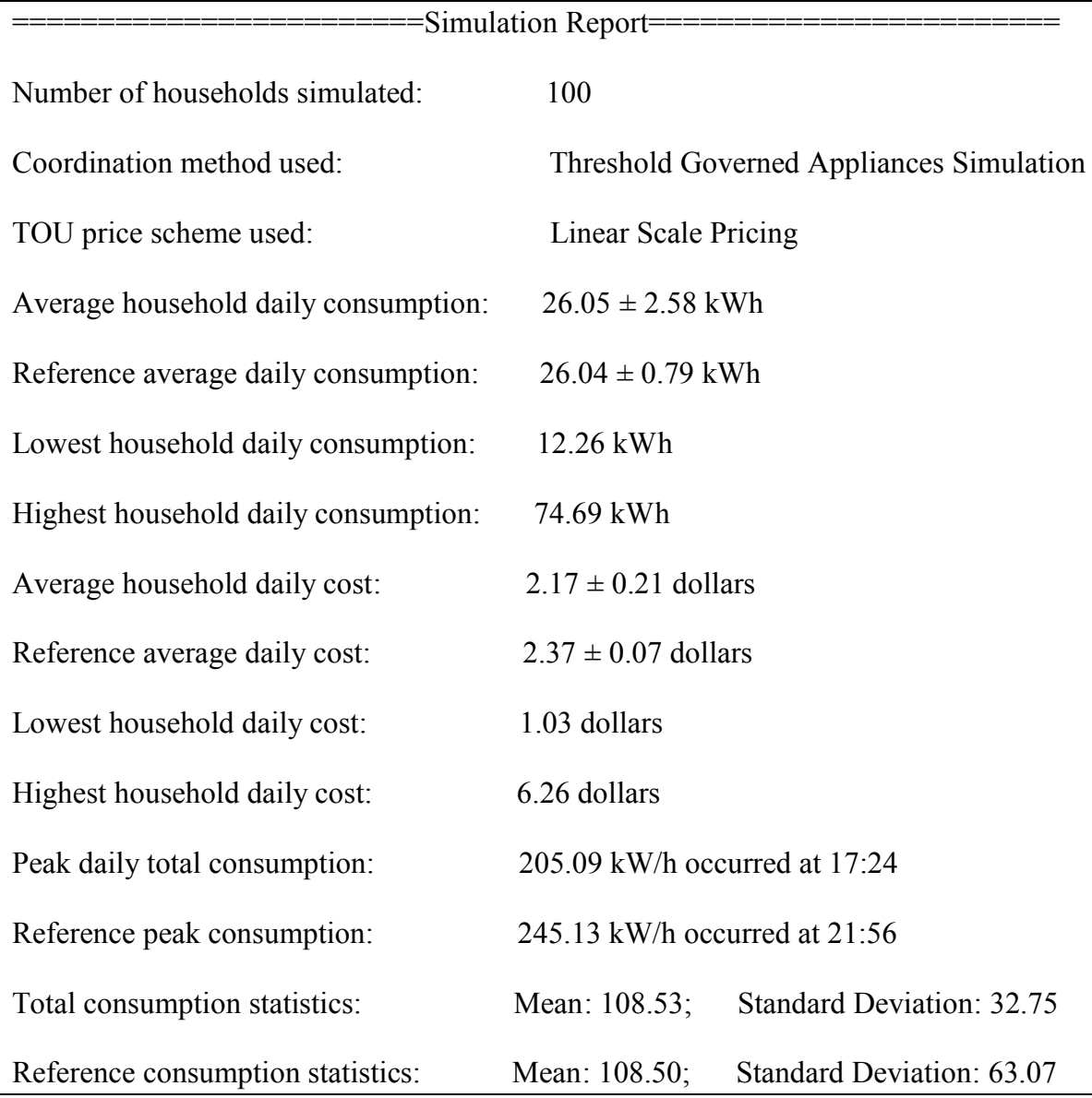

According to the simulation report in Figure 5.13, the simulation yields almost identical amount of consumption, and the corresponding monetary percentage saving is about $9 \%$. To verify the significance, the simulated confidence interval contains the reference one which indicates the insignificance in consumption change. However, the confidence intervals for the average costs are overlapping and the mean of the reference cost falls 
into the interval of the simulated one. Under this situation, a tTest is best used to clarify the significance. The following formula performs the tTest.

Equation 5.3:

$$
\mathrm{t}=\frac{\text { Average }_{1}-\text { Average }_{2}}{\sqrt{\frac{\sigma_{1}^{2}}{\mathrm{n}_{1}}+\frac{\sigma_{2}^{2}}{\mathrm{n}_{2}}}}
$$

In this equation, the $\sigma$ and $n$ are the same standard deviations and number of samples that are explained in Equation 4.10. The built-in Matlab function "ttest2" implements this unpaired two sample tTest. With that function, the result indicates "a rejection of the null hypothesis at the 5\% significance level", which means that the changes from the reference sample to the simulated result is significant. Therefore, similar to the case of programmable appliances, the threshold-controlled appliances are able to achieve higher monetary percentage saving by moving around the consumptions. The reason for the higher monetary percentage saving is the same as explained with Figure 5.11. Also, the threshold-controlled appliances are able to achieve lower peak consumption and better balanced usage of electricity. The main difference from the appliance operation scheduling method versus the threshold controlled coordination method is that the latter one is a more controllable and scalable solution. When more appliances are thresholdcontrolled, the householders do not need to study the consumption patterns to program individual appliance's operation. The time of use price or the demand and supply relationship on the grid is able to govern the appliances' operations automatically. The following subsections will demonstrate the effects of various attributes concerning the efficiency of the threshold controlled coordination method. 


\subsubsection{Effect of Different Price Threshold Settings}

Obviously, the threshold setting affects the result of appliance operations directly. One extreme is that if the threshold is set higher than the maximum linear price the grid can get, there will be no savings or constraints on the appliances' operations; oppositely, if the threshold is set lower than the minimum linear price, none of the controlled appliance is able to operate. Consumers of course want their appliances to operate at the lowest prices, but they also do not want to find that their electrical vehicle is not charged by the morning due to a scheme not being able to meet the preset threshold. In addition to other prevention algorithms such as simply start charging by a deadline regardless of the price, a right balance is of key interest. Due to the complexity of how different households define their priorities of appliance operations, it is hard to define a single goal for the balancing point. For example, some households have to have the dishwashers finish their operation by the breakfast time the next morning, while other households do not have a firm requirement on that or they do not even use the dishwasher. Here, we only concern about the charging of electrical vehicle as the simplest case: assume all households need their electrical vehicle fully charged when they leave to work. Let us further assume that at the latest, a consumer has to leave home at 8:30am. Since the charging cycle is defined as 4.5 hours in this report, by $4: 00 \mathrm{am}$ all the electrical vehicles have to start their charging operation. We define the miss of a charging opportunity as $M_{E V}$ to count how many electrical vehicles could not start charging by 4:00am, and the goal for $M_{E V}$ is 0 . After many tries, a threshold of 8 cent $/ \mathrm{kWh}$ or sometimes 7.5 cent $/ \mathrm{kWh}$ is able to achieve this goal. The following figures are the results from a threshold of 8 cent $/ \mathrm{kWh}$. 

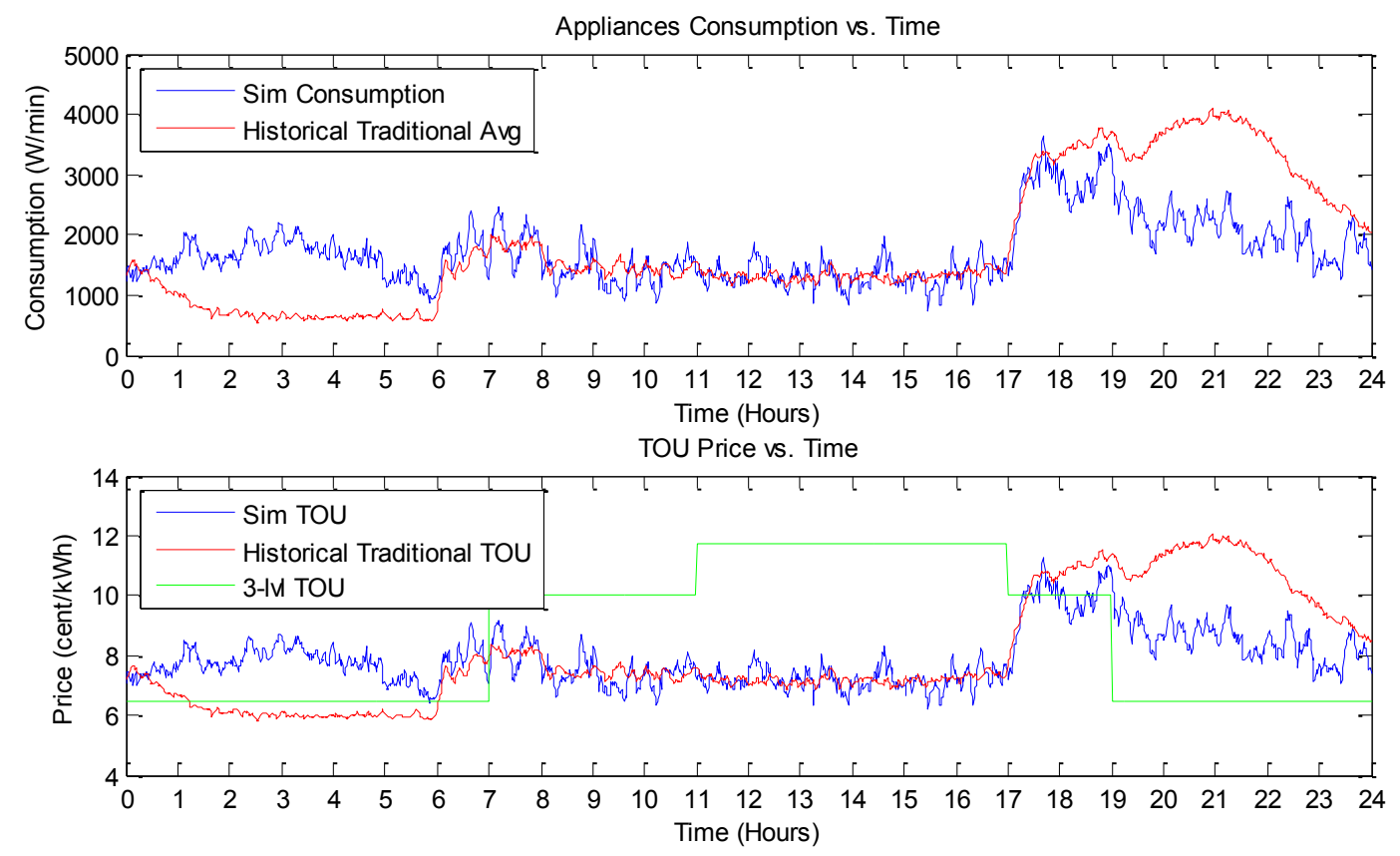

Figure 5.14: Threshold of 8 cent $/ \mathrm{kWh}$ Consumption Plot

As shown in the lower subplot of Figure 5.14, the linear pricing line after 5:00pm drops from around $10 \mathrm{cent} / \mathrm{kWh}$ to around $8 \mathrm{cent} / \mathrm{kWh}$. This is due to the reduction of the corresponding consumption line in the upper subplot. There are two obvious peaks around 5:00pm to 7:00pm which come from the operations of none-shiftable appliances such as stoves and televisions. These appliances are not regulated by the threshold. For the ones that are controlled by the price threshold, their operations are delayed to the next day which is represented by the bulk of extra consumption shown from 0:00am to 6:00am. As stated before, to meet $M_{E V}=0$, all electrical vehicles started their charging operations before 4:00am. As the results of this simulation show, the monetary percentage saving is around $14 \%$, while the consumption has about a $4 \%$ drop due to randomization. The confidence intervals of the average costs are mutually exclusive to indicate the significance. This threshold controlled operation also has a big impact on the peak load 
reduction and a major refinement on the load balancing. The peak consumption drops from $245.13 \mathrm{kWh}$ to $218.30 \mathrm{kWh}$; and the standard deviation drops from 63.07 to 31.57 . Overall, the above results are about the best one can obtain to be able to fully charge the electrical vehicles on time without any other prevention algorithm implemented.

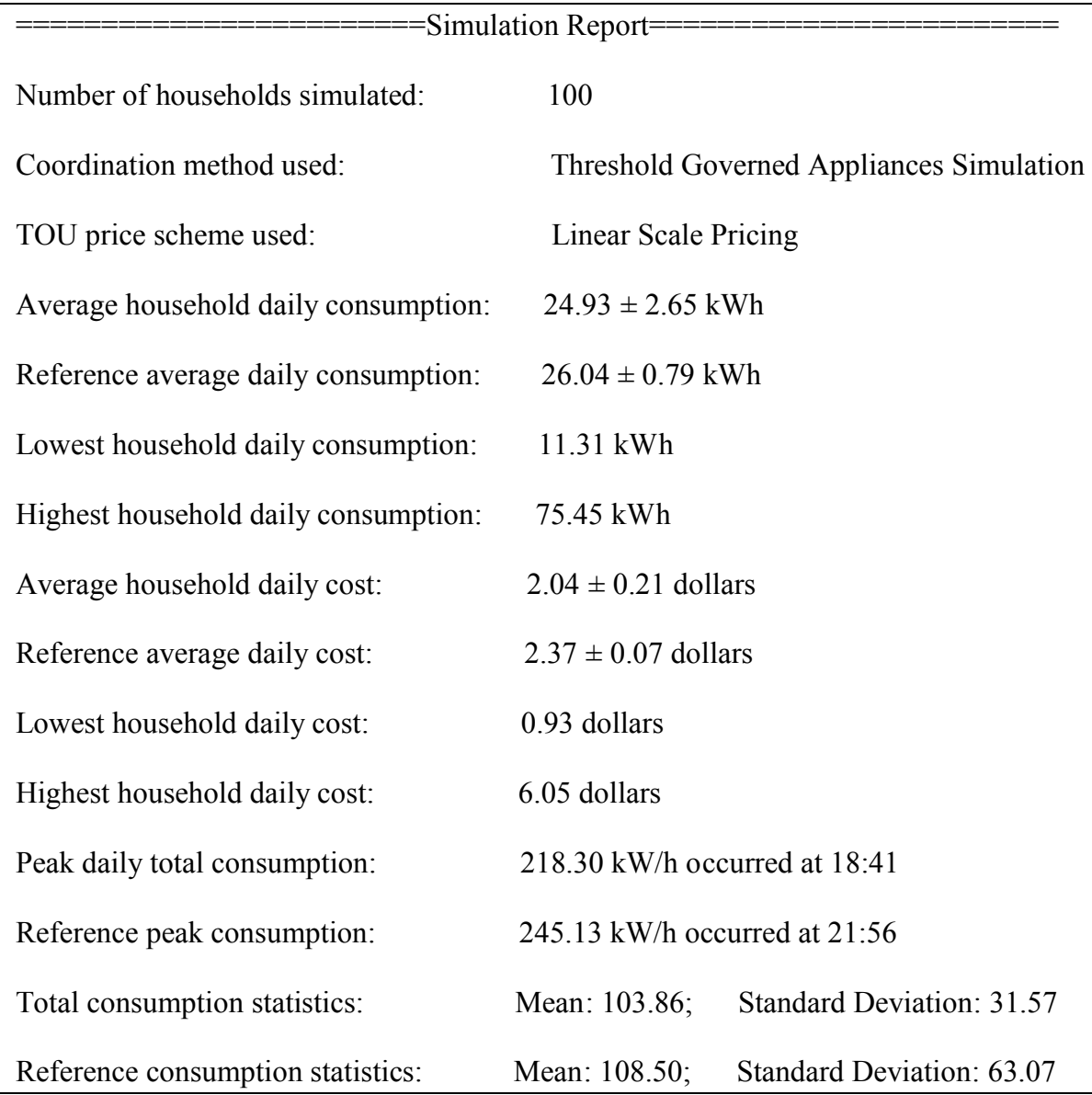

Figure 5.15: Threshold of 8 cent/kWh Consumption Report

\subsubsection{Effect of Pricing Profile Manipulation}

We were able to find a good threshold value to maximize consumer savings while ensuring a fully charged electrical vehicle by the morning. However, such a threshold value is only good for that particular price scheme. As mentioned before, it does not make much sense to use threshold-controlled appliances with flat rate or OnPeak- 
MidPeak-OffPeak pricing scheme. However, that conclusion is only applicable to a static price profile that is pre-known to consumers and not subject to change. Threshold governed coordination is still valid to respond to peak control purpose price changes. For example, assume that a flat rate of 7 cent $/ \mathrm{kWh}$ is normally used, and all households' threshold is set to 8 cent $/ \mathrm{kWh}$. This threshold does not take effect as it is never met at the flat rate. Assume one day excessive consumption is detected on the grid, and thus the utility changes the price to 9 cent $/ \mathrm{kWh}$ to force the controlled appliances to turn off. The following figures illustrate the effect.
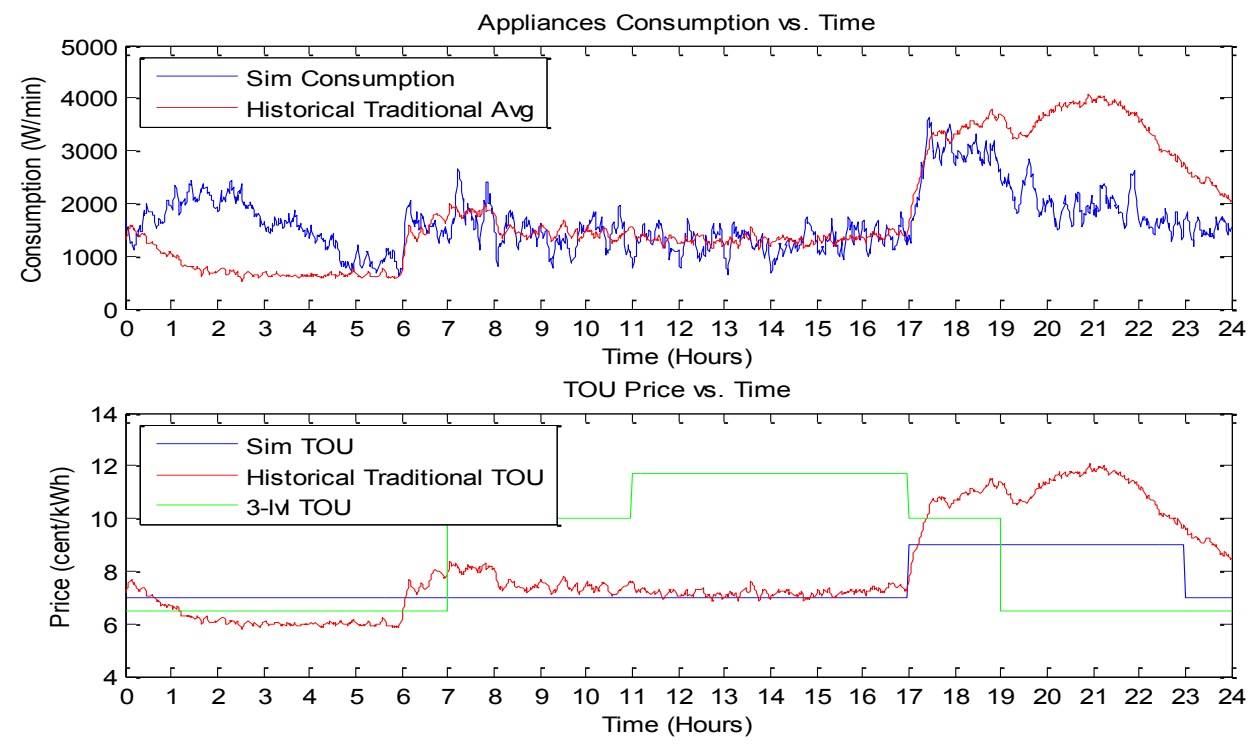

Figure 5.16: Price Profile Scaling Effect Plot

As shown in Figure 5.16, the price change takes effect immediately and turns off all controlled appliances until 11:00pm. At that point, all turned off appliances resume their normal operation and try to start. The large bulk of consumption is effectively shifted from evening to the next morning. This effect is similar to the linear pricing case except that the consumption cap is not well controlled. The peak of the morning consumption is 
higher than that of the evening. Nonetheless, the simulation report in Figure 5.17 still displays a major drop in consumption standard deviation. The value of 32.82 is just slightly higher than 31.57 in Figure 5.15. Therefore, in general, the threshold controlled appliances are also useful to respond to governmental consumption control. This type of functionality already exists in another form of control. Utility companies are able to adjust participating households' thermostats to regulate the consumption behavior.

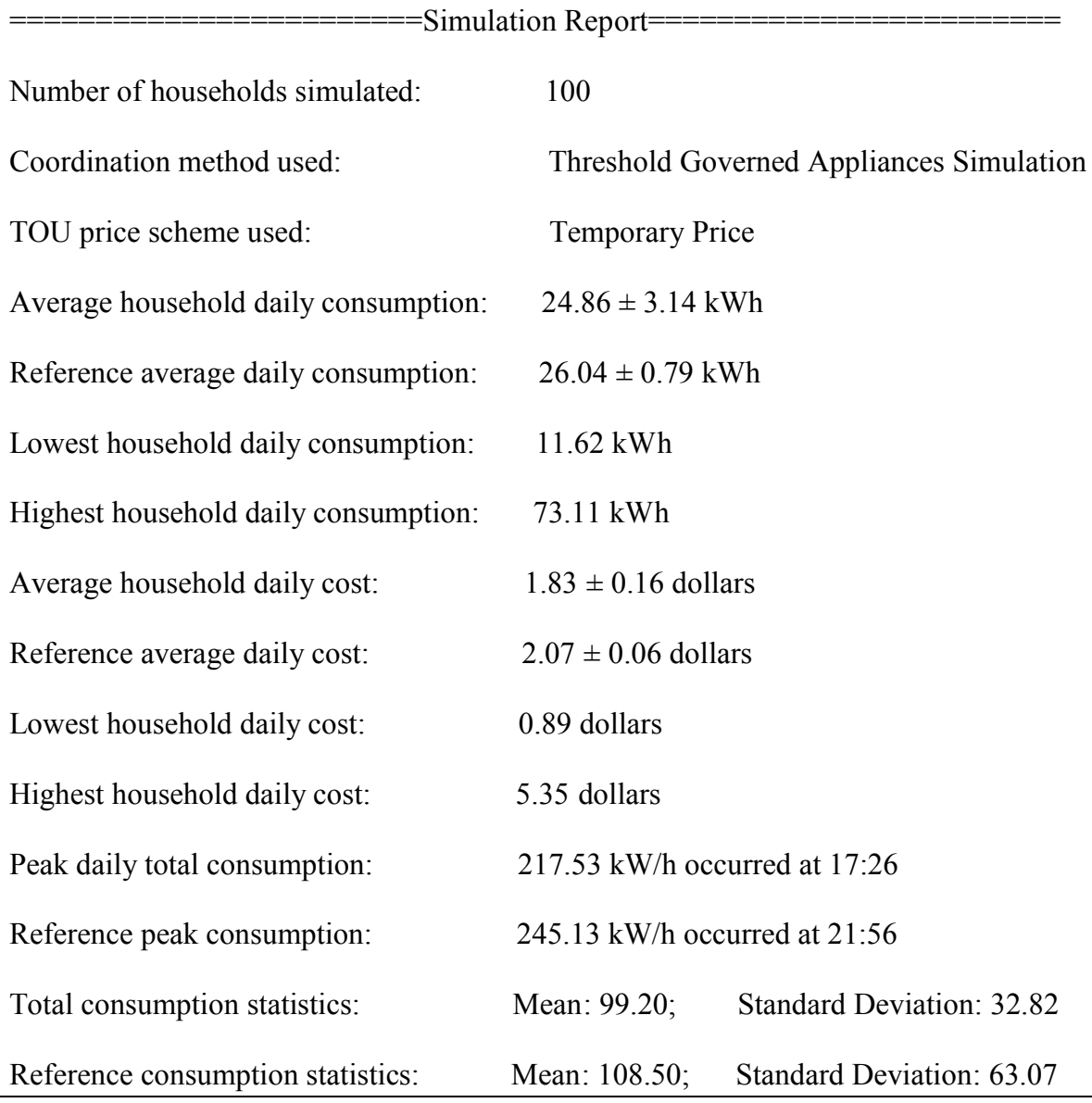

Figure 5.17: Price Profile Scaling Effect Report

Overall, threshold-controlled appliances are best to operate under dynamic pricing schemes such as the linear pricing scheme. Since the price is always proportional to the consumption, it is able to attract most participation. Utility companies can scale up/down 
the price difference to set proper incentives. The current price difference is about 12 cent $/ \mathrm{kWh}-6.5 \mathrm{cent} / \mathrm{kWh}=5.5 \mathrm{cent} / \mathrm{kWh}$. If the price range is scaled from 5 cent $/ \mathrm{kWh}$ to $13.5 \mathrm{cent} / \mathrm{kWh}$, that would entice consumers to take more advantage of thresholdcontrolled operation.

\subsubsection{Effect of Communication Failures}

One key difference from the threshold governed operation to the previous two coordination methods is the need of communication between the control center or utility company who regulates the time of use price calculation and distribution. It is therefore a key element of the simulation framework to capture the behavior of this activity. Nonetheless, the current implementations of the framework only focus on the behavioral level effects as explained in Chapter 3. The following simulation results demonstrate the effect of losing $50 \%$ of the information packets sent from households to the control center to report individual consumptions.
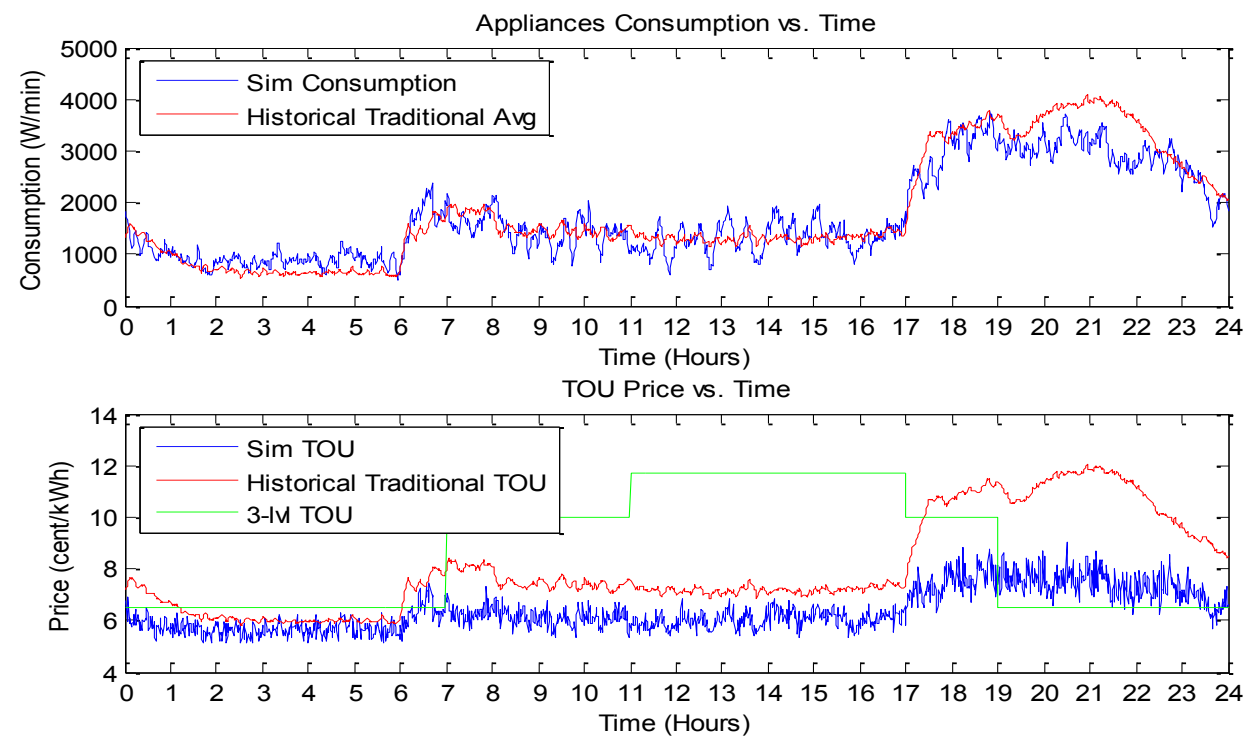

Figure 5.18: Communication Failure Plot 


\begin{tabular}{|c|c|}
\hline Number of households simulated: & 100 \\
\hline Coordination method used: & Threshold Governed Appliances Simulation \\
\hline TOU price scheme used: & Linear Scale Pricing \\
\hline Average household daily consumption: & $24.87 \pm 2.45 \mathrm{kWh}$ \\
\hline Reference average daily consumption: & $26.04 \pm 0.79 \mathrm{kWh}$ \\
\hline Lowest household daily consumption: & $13.36 \mathrm{kWh}$ \\
\hline Highest household daily consumption: & $72.74 \mathrm{kWh}$ \\
\hline Average household daily cost: & $2.15 \pm 0.21$ dollars \\
\hline Reference average daily cost: & $2.37 \pm 0.07$ dollars \\
\hline Lowest household daily cost: & 1.13 dollars \\
\hline Highest household daily cost: & 6.79 dollars \\
\hline Peak daily total consumption: & $227.49 \mathrm{~kW} / \mathrm{h}$ occurred at $19: 50$ \\
\hline Reference peak consumption: & $245.13 \mathrm{~kW} / \mathrm{h}$ occurred at $21: 56$ \\
\hline Total consumption statistics: & Mean: $103.62 ; \quad$ Standard Deviation: 51.18 \\
\hline Reference consumption statistics: & Mean: $108.50 ; \quad$ Standard Deviation: 63.07 \\
\hline
\end{tabular}

Figure 5.19: Communication Failure Report

As shown in the lower subplot of Figure 5.18, the values of the simulated time of use price line (blue line) is about half of what it is actually supposed to be. This is due to the fact that the control center does not have the consumption information from half of the simulated households at any given time. Only half of the consumptions are counted for price calculation. As the result, the time of use price is almost always lower than the threshold and thus not taking effect. However, this result is based on the assumption that the consumptions are collected from individual households. In reality, utility companies are able to observe the load condition directly from the supply side. 
The next experiment is used to see the effect of TOU price distribution communication failures. However, the effect of that is invisible in the simulation plots because i) there is no communication curve in the plot, and ii) there is no visible effect in both consumption and the price. The following section will explain the reasons.

\subsubsection{Effect of Different TOU Price Announcement Frequency}

For all previously demonstrated results, the TOU price is announced at every minute. That would obviously introduce a large amount of communication on the information grid. This is not a desired result. It is thus interesting to see what the tradeoffs are if the frequency of TOU price distribution is reduced. The following example announces the TOU price every 30 minutes instead of every minute.
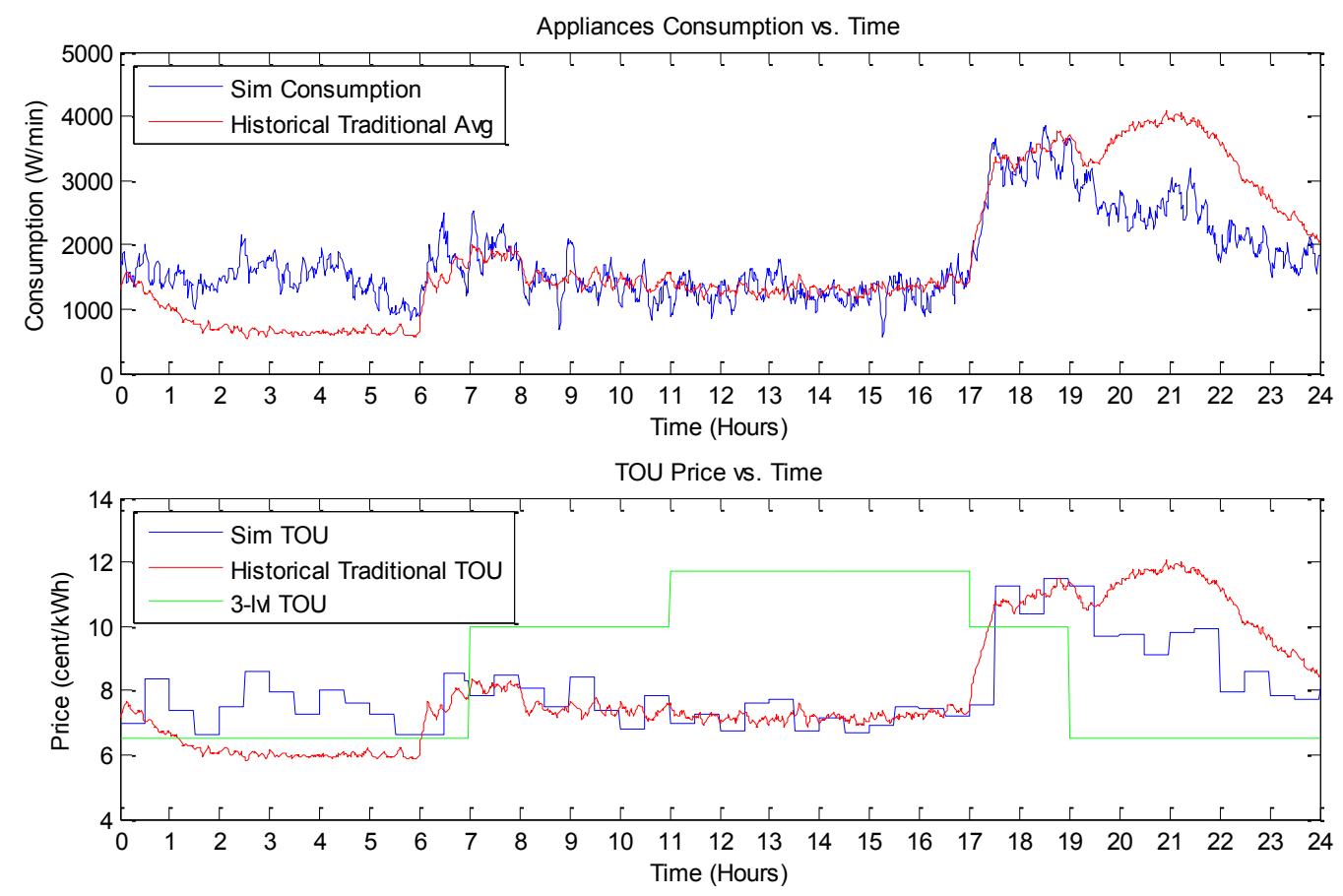

Figure 5.20: Effect of Decreasing the TOU Price Distribution Frequency Plot 


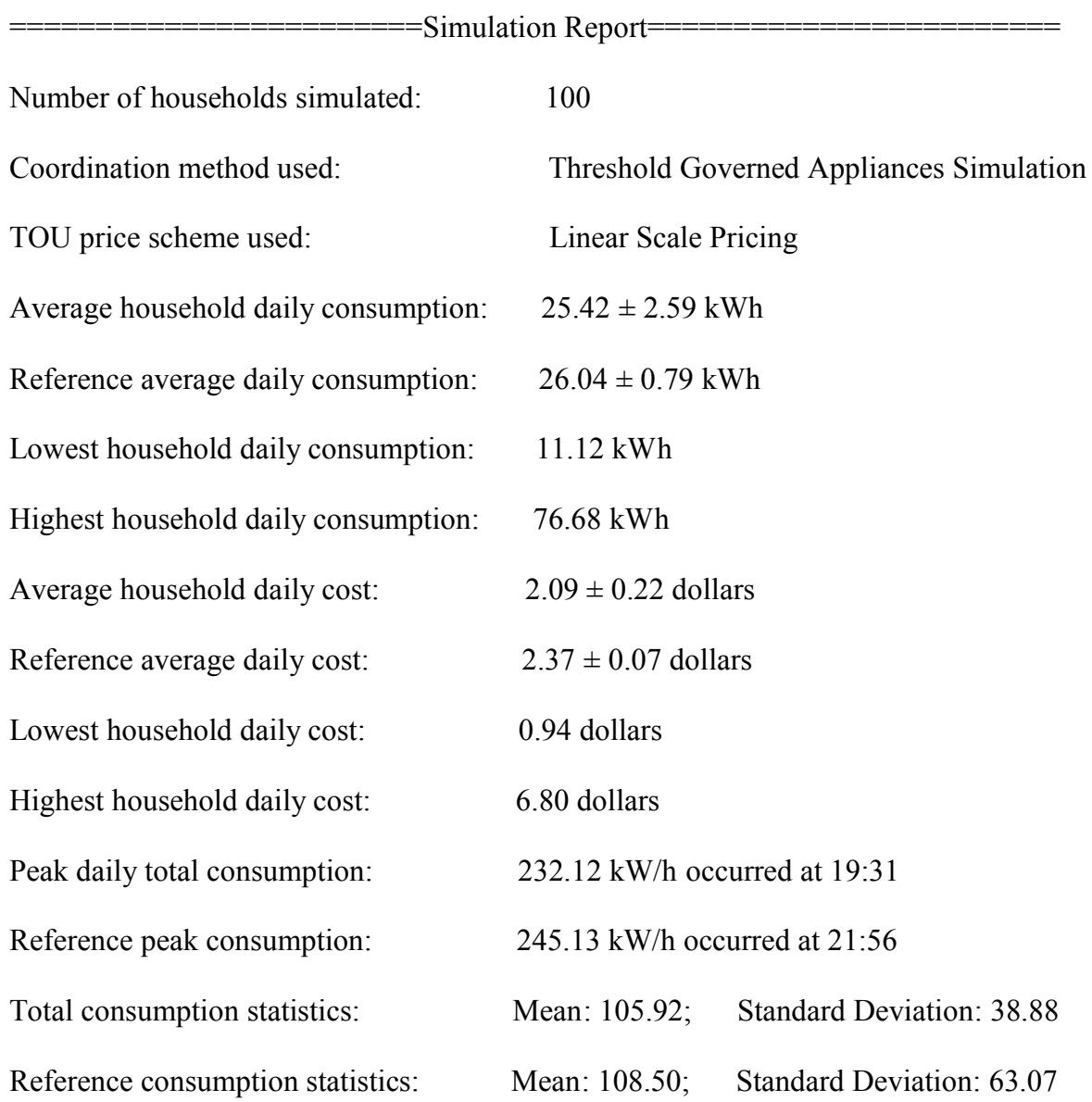

Figure 5.21: Effect of Decreasing the TOU Price Distribution Frequency Report

As shown in the lower subplot of Figure 5.20, the time of use prices are announced every 30 minutes. Effectively speaking, the appliances will use the same price for 30 minutes. As shown, although a lot of information is lost in the price signal, the overall shape of the price profile still follows the consumption profile. The defined threshold still takes effect and the overall consumption behavior looks not much different from Figure 5.14. Comparing the simulation results shown in Figure 5.15 and Figure 5.21, they are very close except the later simulation yields a slightly higher overall consumption. Overall, 30 minutes of TOU price hold time does not affect the consumption behavior greatly. The 
next chapter will provide a case study to determine the optimal pricing scheme along with other goals.

Based on these results, it is not hard to understand why TOU distribution communication failures would not impact the consumption behavior much. Even in the case that $50 \%$ of the distribution packets are lost, that is only equivalent to sending the TOU price every 2 minutes on average. This difference is negligible.

To conclude, threshold-controlled appliances can work well with a linear pricing scheme to achieve good monetary percentage saving to reward consumers. It is also able to benefit the overall power grid since the consumption pattern is more stable. Among the demonstrated coordination methods, the threshold-controlled operation scheme seems to be the best. The next chapter is a case study example to illustrate the usage of the simulation framework. 


\section{Chapter 6: Case Study}

In this chapter, we demonstrate, via a case study, how to use the proposed simulation framework to explore policy alternatives/pricing strategies for the smart grid.

\subsection{Introduction to the Case Study}

As shown in the previous chapter, a dynamic pricing scheme is most suitable for the smart grid infrastructure, able to realize the value of the integrated information network. It reflects the load condition on the grid, and sometimes also reflects regulatory needs and/or designed incentives. It is a very natural means of representation for the value of energy at different times. Therefore, it is very beneficial for governments and utility companies to implement dynamic pricing schemes, which will be the focus of this case study.

To react to the dynamic pricing scheme, the previous chapter has illustrated that a real time threshold controlled operation is the best choice over the implemented coordination schemes. We will thus employ such a coordination scheme in the case study. We study the effect of changing the threshold settings on households' operations and also on the overall power grid.

Imagine that a power utility company is seeking for a TOU price plan which would achieve the best balance in power usage. In doing so, the waste of generated energy is minimized, and also mechanical damages to the generators are reduced because the machines do not need to be repeatedly turned on and off to satisfy the unstable demand 
from the power grid. For simplicity, let us assume that the power utility company also controls the threshold settings of individual appliances in all households. This assumption is an extension to real life programs that provide utility companies with some control of households' thermostat settings. When power is in peak demand, a utility company is able to adjust all participating households' thermostat to slightly adjust the temperature settings to lower the overall energy consumption [64]. We extend this model, giving the utility company controls of all capable appliances instead of just the thermostats. To further simplify the problem, we also assume that the same threshold price is applied to all households,. Based on this simple model, we are interested to see the effects on the power grid caused by changes to these threshold settings, and to decide the best threshold to use. Overall, our case study goal is to find an optimal threshold setting that would achieve maximum power grid consumption stability. At the same time, this threshold setting cannot sacrifice consumers' comfort.

\subsection{Simulation Results and Discussion}

For all the simulations presented in this chapter, 100 households are used as the sample size. 15 electrical vehicles are included in the simulations because they are the appliance most amenable to operation shifting. The time of use price announcements are made every minute. There is no communication failure in the simulations. Under these settings, the threshold price is adjusted iteratively from $12 \mathrm{cent} / \mathrm{kWh}$ down to 6 cent $/ \mathrm{kWh}$. The table below summarizes the simulation results. 
Table 6.1: Threshold Setting Iteration Table

\begin{tabular}{|c|c|c|c|c|c|c|c|}
\hline $\begin{array}{c}\text { Threshold } \\
\text { Setting } \\
\text { (cent/kWh) }\end{array}$ & $\begin{array}{c}\text { Average } \\
\text { Consumpt- } \\
\text { ion } \\
\text { (kWh) }\end{array}$ & $\begin{array}{c}\text { Consumpt- } \\
\text { ion } \\
\text { Saving } \\
(\mathbf{\%})\end{array}$ & $\begin{array}{c}\text { Average } \\
\text { Cost } \\
\text { (dollars) }\end{array}$ & $\begin{array}{c}\text { Monetary } \\
\text { Saving } \\
(\%)\end{array}$ & $\begin{array}{c}\text { Peak } \\
\text { Consumpt- } \\
\text { ion } \\
\text { (kWh) }\end{array}$ & $\begin{array}{c}\text { Missed EV } \\
\text { Charging } \\
\text { Count }\end{array}$ & $\begin{array}{c}\text { Standard } \\
\text { Deviation }\end{array}$ \\
\hline $\begin{array}{c}\text { Reference } \\
\text { (No } \\
\text { Threshold) }\end{array}$ & 26.04 & N/A & 2.37 & N/A & 245.13 & 0 & 63.07 \\
\hline 12 & 26.38 & -1.3 & 2.36 & 0.4 & 269.77 & 0 & 57.30 \\
\hline 11 & 26.22 & -0.1 & 2.30 & 3.2 & 235.26 & 0 & 51.24 \\
\hline 10 & 26.31 & -1.0 & 2.25 & 5.3 & 216.75 & 0 & 42.08 \\
\hline 9 & 26.23 & -0.7 & 2.20 & 7.5 & 231.69 & 0 & 33.95 \\
\hline 8 & 24.95 & 4.2 & 2.05 & 13.8 & 223.13 & 0 & 32.44 \\
\hline 7 & 23.13 & 11.2 & 1.84 & 22.6 & 199.79 & 3 & 29.06 \\
\hline 6 & 21.36 & 18.0 & 1.68 & 29.17 & 216.40 & 6 & 34.88 \\
\hline
\end{tabular}

There are eight columns in Table 6.1, our major goal is to find the optimal threshold setting to minimize the standard deviation of the consumption profile. Recall that standard deviation measures the disparity of a data set. A small standard deviation means that all the data in the data set are tightly centered around the mean of the data set. In our case, a small standard deviation indicates a stable consumption profile on the power grid, which is what the utility companies are seeking. As shown in the last column, the standard deviation drops gradually, and reaches its lowest value of 29.06 at a threshold price of $7 \mathrm{cent} / \mathrm{kWh}$. After that, the standard deviation increases to 34.88 at a threshold of $6 \mathrm{cent} / \mathrm{kWh}$. This increase in standard deviation is shown in Figure 6.1 and Figure 6.2 below. These two figures together illustrate the results shown in Table 6.1. Due to the high density of lines, the overall picture is divided into two separate figures for better visibility. The black lines in both figures are the reference lines with no coordination applied. In Figure 6.1, a threshold of $12 \mathrm{cent} / \mathrm{kWh}$ puts very little restriction on the consumption and therefore results in the highest peak at around 22:30 in the magenta colored line. After that, with every drop in the threshold setting, the consumptions during the evening are pushed lower, and shifted to overnight. At the price of $7 \mathrm{cent} / \mathrm{kWh}$, the 
consumption line (blue line in Figure 6.2) becomes largely leveled with the reference consumption during the day between 8:00 to 17:00. Therefore, the blue line achieves the lowest standard deviation. After this point, when the threshold is dropped further to 6 cent $/ \mathrm{kWh}$, the green line falls below the 7 cent $/ \mathrm{kWh}$ line between midnight and 6:30 am, because the consumption is suppressed too much due to the tight threshold. This introduces a negative effect in reducing standard deviation. This is therefore the reason for the increase shown in Table 6.1.

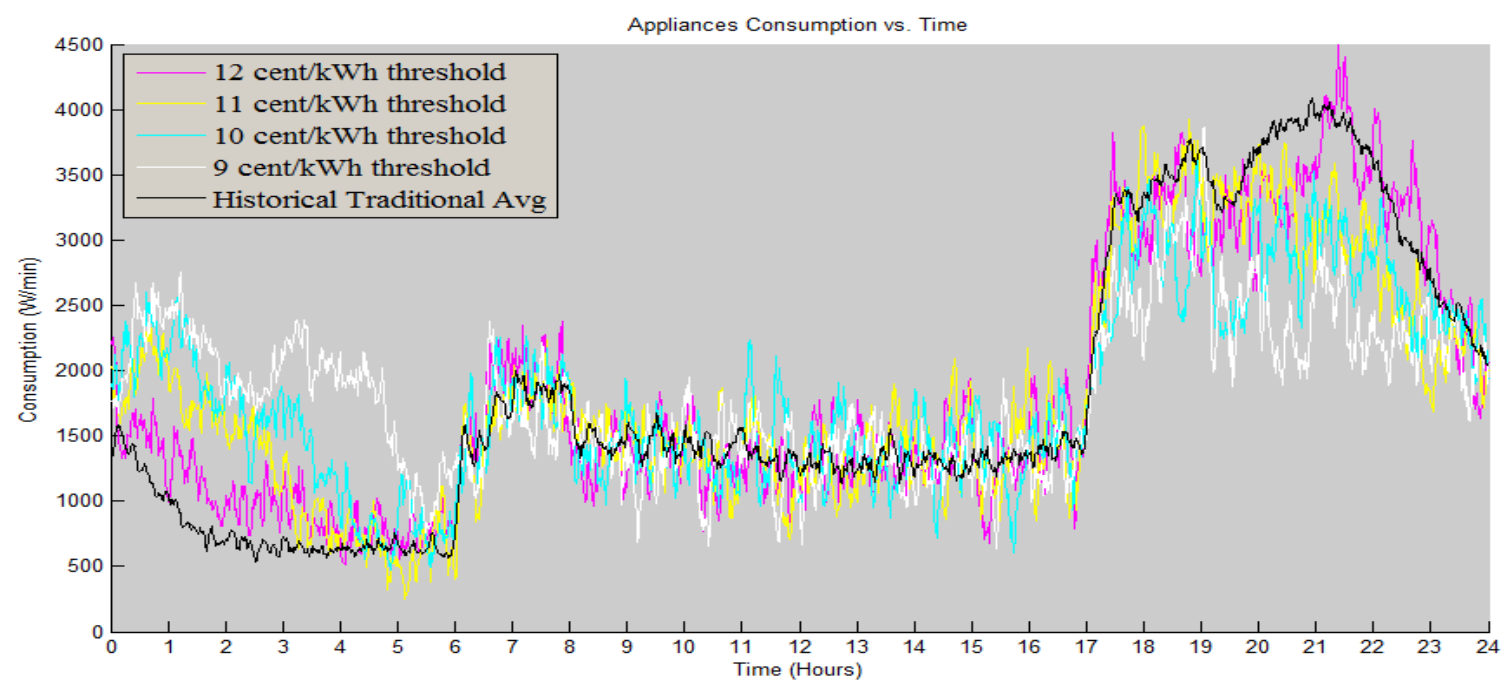

Figure 6.1: Threshold Iterating Plot (a)

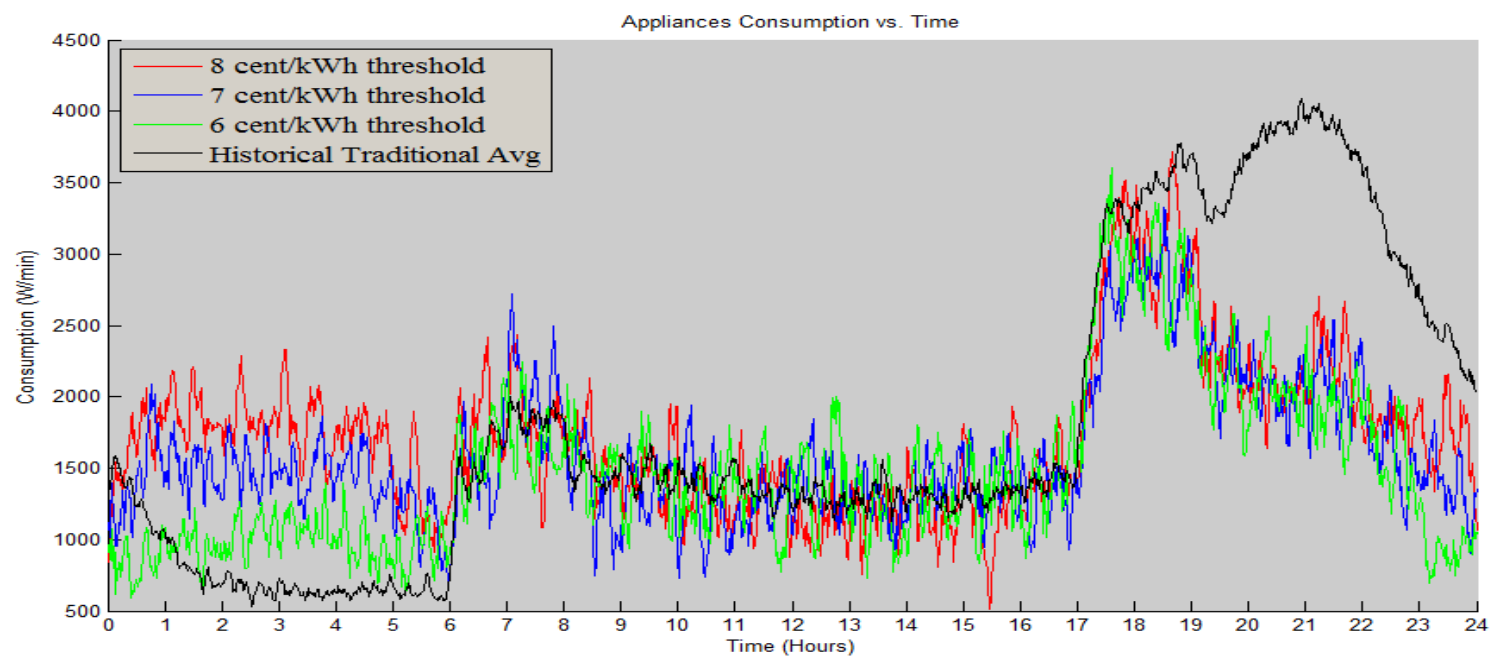

Figure 6.2: Threshold Iterating Plot (b) 
However, this does not mean we found the optimal value or at least not a desired threshold setting to meet the satisfaction of residential consumers. Looking at the "Consumption Saving" column of Table 6.1 , there is a $11.2 \%$ of energy saving at 7 cent $/ \mathrm{kWh}$ compared to the reference consumption. However, the threshold controlled operation coordination method does not reduce energy consumption, but merely shifts the consumption. This $11.2 \%$ reduction of consumption really comes from the miss of opportunities for certain appliances to start their operation. One important appliance that should not miss its operation is the charging of electrical vehicles, and that is why a column in Table 6.1 is dedicated for this measurement. Looking at the "Missed EV Charging Count" column in Table 6.1, with a reduction of $11.2 \%$ in energy consumption, the simulation result shows that three electrical vehicles miss their chance to get charged. Therefore, a threshold price of $7 \mathrm{cent} / \mathrm{kWh}$ is definitely not an optimal solution we are looking for to satisfy customers. Returning to Table 6.1, the consumption percentage savings for thresholds starting at 12 cent $/ \mathrm{kWh}$ down to 8 cent $/ \mathrm{kWh}$ are all within plus/minus 5\%. A negative saving means an overall consumption increase, due to the arbitration mechanism built into the simulation framework. At a threshold of 8 cent $/ \mathrm{kWh}$, the overall consumption was reduced by $4.2 \%$. This percentage change in consumption is larger in magnitude than the previous one. However, this change is still statistically insignificant. The confidence of average consumption at $8 \mathrm{cent} / \mathrm{kWh}$ is $24.95 \pm 2.29 \mathrm{kWh}$, and the reference consumption is $26.04 \pm 0.79 \mathrm{kWh}$. This $4.2 \%$ of reduction in energy consumption is introduced by the arbitration process of the simulation, and all the electrical vehicles are indeed charged overnight. At a threshold of $8 \mathrm{cent} / \mathrm{kWh}$, the average daily energy cost is 2.05 dollars. It is the lowest value from 12 cent $/ \mathrm{kWh}$ down to 
$8 \mathrm{cent} / \mathrm{kWh}$. Again the values for $7 \mathrm{cent} / \mathrm{kWh}$ and $6 \mathrm{cent} / \mathrm{kWh}$ are eliminated from the candidates. The monetary percentage saving is $13.8 \%$, which is the highest rate of return among the candidates. The peak consumption value is the second lowest among the candidates. This result is purely due to simulation arbitration. Since this value is still lower than the peak value of the reference consumption curve, it is acceptable. Therefore, overall, 8 cent $/ \mathrm{kWh}$ is the optimal threshold we are looking for. Let us now look at the chosen optimal solution in the following figure.

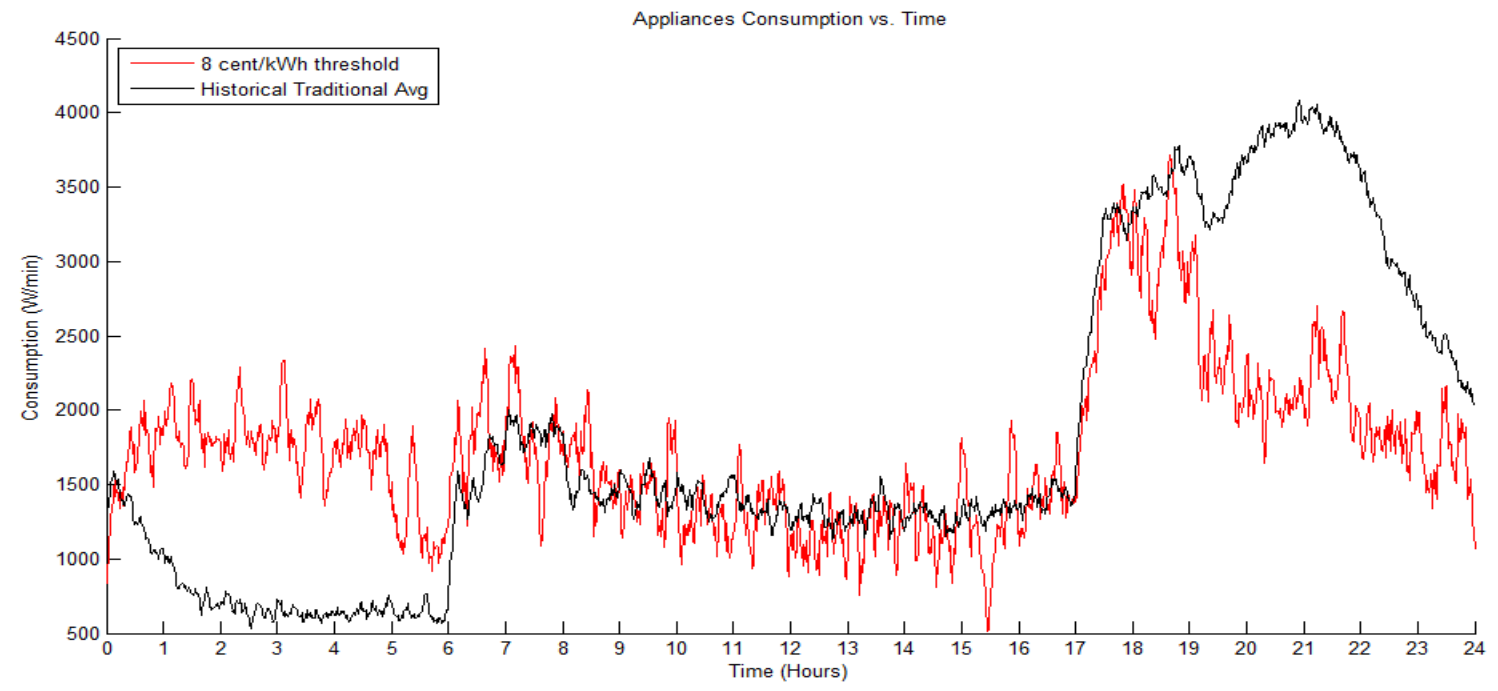

Figure 6.3: Optimal Threshold Plot

As shown in Figure 6.3, the consumption profile of the chosen optimal threshold is largely following a flat trend on average. The only exception happens between 17:00 and 23:00. This evening peak results from the none-shiftable appliances, which are not affected by the price threshold control. This peak can be reduced by more energy efficient appliances, or it can be covered by renewable energies. 
To conclude, we found an optimal threshold setting to minimize the consumption dispersion without sacrificing customer comfort using the implemented simulation framework. We demonstrated the flexibility and comprehensive nature of the simulation framework. It can be adjusted to meet different needs from different users and projects. 


\section{Chapter 7: Conclusion and Future Work}

This last chapter recaps the goal of this research project, and summarizes the key contributions and findings towards these goals. Lastly, some ideas on further works or future research topics to extend the existing content of this research will be given.

\subsection{Thesis Contributions}

In this project, we started out by researching the current state of art in the smart grid development happening around the globe. We learnt the great benefits and potential values of this evolutionary technology advancement. While great excitements are shared among industries and researchers, some difficulties in deployment and some levels of resistance are seen in the project advancement. Consumers lack knowledge about smart grid in general; some product developers offer inadequate solutions to the smart grid market; regulation bodies are battling in the standardization activities; and researchers are lacking a base of comparison for their innovations.

After identifying these problems, we determined our research project to offer a flexible simulation framework that can be used and extended for different needs in the smart grid field. Consumers can use this simulation framework to learn their individual consumption behavior and/or to compare different products provided in the market; product developers and researchers can use this simulation framework to test the impacts and efficiencies of their innovations; regulatory bodies can use this simulation framework to examine any proposed plans before project roll out. 
With these goals in mind, we designed a modular simulation framework that puts the emphasis on the residential consumer power demand side of the power grid model, and further implemented a prototype of the proposed framework. We first modeled each major household appliance's consumption behavior in terms of their instant power consumption and operation period, and built an appliances consumption library. The main simulation process then uses the consumption library to generate the consumption profiles of a specified number of households. Various simulation results are kept after the simulation for analysis. A simulation report and a simulation plot are automatically generated to summarize the key results from the simulation. We validated the simulation results by comparing our base case to published records and with gathered data from a small survey. The simulation results are able to truthfully reflect the power consumption behavior in reality.

After validating the prototype, we used it to test out some simple appliance coordination methods. We therefore implemented an operation scheduling algorithm and a price threshold controlled operation coordination algorithm. We provided detailed analysis and discussions to compare the impact and efficiencies of different coordination systems. We realized that the value or the worth of electricity dynamically changes over the course of a day depending on the demand and availability of electricity at the moment. Dynamic pricing schemes are adequate to represent this change in value. We therefore employed a linear pricing scheme in later studies. Under the linear pricing scheme, the price threshold controlled coordination method is better in reacting to the change of price signal. Some benefits from such coordination method includes automatic adjustment to price changes, 
households are able to achieve monetary savings even while consuming the same amount of total power, and it is a better approach in terms of load balancing. Electrical vehicles benefit the most from the threshold controlled system. The impact of heavy consumption demand caused by the introduction of electrical vehicles is handled very well by the threshold controlled method. The consumption profile is able to achieve an automatic balance without much customer interventions. By adjusting the threshold setting, we were able to find an optimal value that is able to achieve the best power balance on the overall grid, without sacrificing consumers' comfort.

Overall, we accomplished the goals we set out to do for this particular research project. We successfully finished the design of the proposed simulation framework. We implemented and validated a prototype of the design. We used the prototype to compare two simple appliance coordination methods and provided detailed analysis based on the simulation results. We demonstrated our reasoning on why a dynamic pricing scheme, coupled with a threshold controlled coordination system, works better and is able to realize the true value of the smart grid infrastructure. We believe our proposed simulation framework is capable to benefit smart grid developments.

\subsection{Future Work}

Although we did achieve a satisfactory result in our research project, there are still much more that can be done to refine and/or to extend this project. There are two major directions to the project continuation. One is to enhance the current simulation framework. Below we list a few of the possible enhancements: 
- As mentioned a few times in this thesis, we do not yet have a model for the power supply side of the power grid model. This is very important for the integrity of a practical demand-supply analysis. Only after realizing such a model we are able to see the true relationship or the true worth of energy at different times. Time of use price profile calculation should be highly dependent on the power supply model. Other aspects such as a peak load reduction study and a load balancing study are also directly dependant on this.

- Similarly, we are also lacking the none-residential part of the power consumption of the demand side model. As a general sense, the industrial or none-residential power consumption is much larger than the residential power consumption. Unfortunately, unlike the residential consumption, which is relatively simple to model, industrial consumption model is much more complex. Different types of industry may exhibit completely different consumption characteristics. Naturally, it means specific models need to be developed for different types of industry, and this is even more crucial if consumption coordination is desired among the industries. Perhaps a starting point in this regard is to find meaningful consumption histories from the overall power grid to extract related information as a whole, instead of implementing individual models for different industries.

- Currently, our proposed framework is a single day based simulation tool. However, often a periodic or seasonal simulation is desired for some needs. Although this kind of demand is able to be accomplished by running the simulation multiple times to arrive at the desired aggregate of average result, it is still desirable to be able to put in some characteristics into the simulations that 
span multiple days. For example, if a study needs a simulation for residential simulation of a complete week, there might be a pattern or difference in each day's consumption. Some people may stay at home longer over the weekend, or some others may enjoy outdoor life over the weekend. If this type of characteristic can be built into the simulation framework, it will be much better in reflecting reality.

- The information communication system implemented in the simulation framework prototype was kept at a minimal level. No real communication protocol was used. With this bare minimal communication implementation, the effects of communication error, latency, and failure were found to be insignificant in most cases. We were not able to get a real taste of how much throughput and bandwidth is required for the communications. The downstream TOU price announcement seems to be light weight when FM broadcasting is available. However we do not have a realistic model of upstream consumption and status reporting communication. More explorations are needed in this regard.

- We have put in a significant amount of effort in software code validation and testing, however we do not claim a perfect code or an optimal code. One field that might be improved is the simulation speed. Matlab does not require compilation of the codes which generally means the runtime speed is not optimized. This might be solved by converting the codes into $\mathrm{C}++$ or Java. However, in doing so, one would lose the powerful matrix manipulation and calculation functionalities provided by Matlab. There are some tradeoffs to judge. Also, Matlab is not truly 
an object oriented program language, inheritance and polymorphism is hard to achieve. Maybe some alternative tool is needed.

The second direction in future work is basically open ended. What our simulation framework provides is a tool, a further development basis. It is designed to allow different users to extend it in order to meet their specific needs and goals. Below is just a small subset of what our simulation framework can be used to do:

- Appliance coordination logics implementation and comparison - This was actually the starting point of our research project. We were interested in analyzing different coordination logics but realized the lack of base building blocks and comparison tool. We then turned around to provide this building block for further research and development.

- Renewable energy integration - With this simulation framework, the impacts of renewable energy integration can be studied more closely and more easily. Both individual household consumption behavioursanalysis and overall metro scale power grid studies can be achieved with the proposed simulation framework. This can be very handy in power balancing studies.

- Public lecture and demonstration - One obstacle that smart grid advancement needs to overcome is to obtain consumer acceptance. With this simulation tool, and perhaps with some good graphics, government or power utility companies can present and demonstrate to the public how the smart grid can benefit the society. They can go as detailed as getting customers to enter their own usage and 
illustrate how to save money with respect to an individual situation. One can even profit from commercialize the simulation framework into a software product.

- Standardization accelerant - As mentioned in Chapter 2, one major battle in smart grid development is the standardization activities. Different groups and organizations want to arrive at a common point to maximize the interoperability. However, the decision is not trivial but rather complex. One problem is again a lack of a base case for comparison between different protocols and technologies. If the proposed simulation framework is able to be used to help speed up the process, it will make a significant contribution to the smart grid development as a whole.

- Security analysis and/or risk management - One major topic in smart grid development is the security of the private information being communicated. Although this type of research has its very own domain of study, our simulation framework may aid in the study of the impacts if breaches occurs in security. What happens to grid stability, for example, if a hacker commands all connected appliances to run at their maximum power. This can be an interesting topic. 


\section{Appendices}

\section{Appendix A - Appliance Consumption Table Implementation}

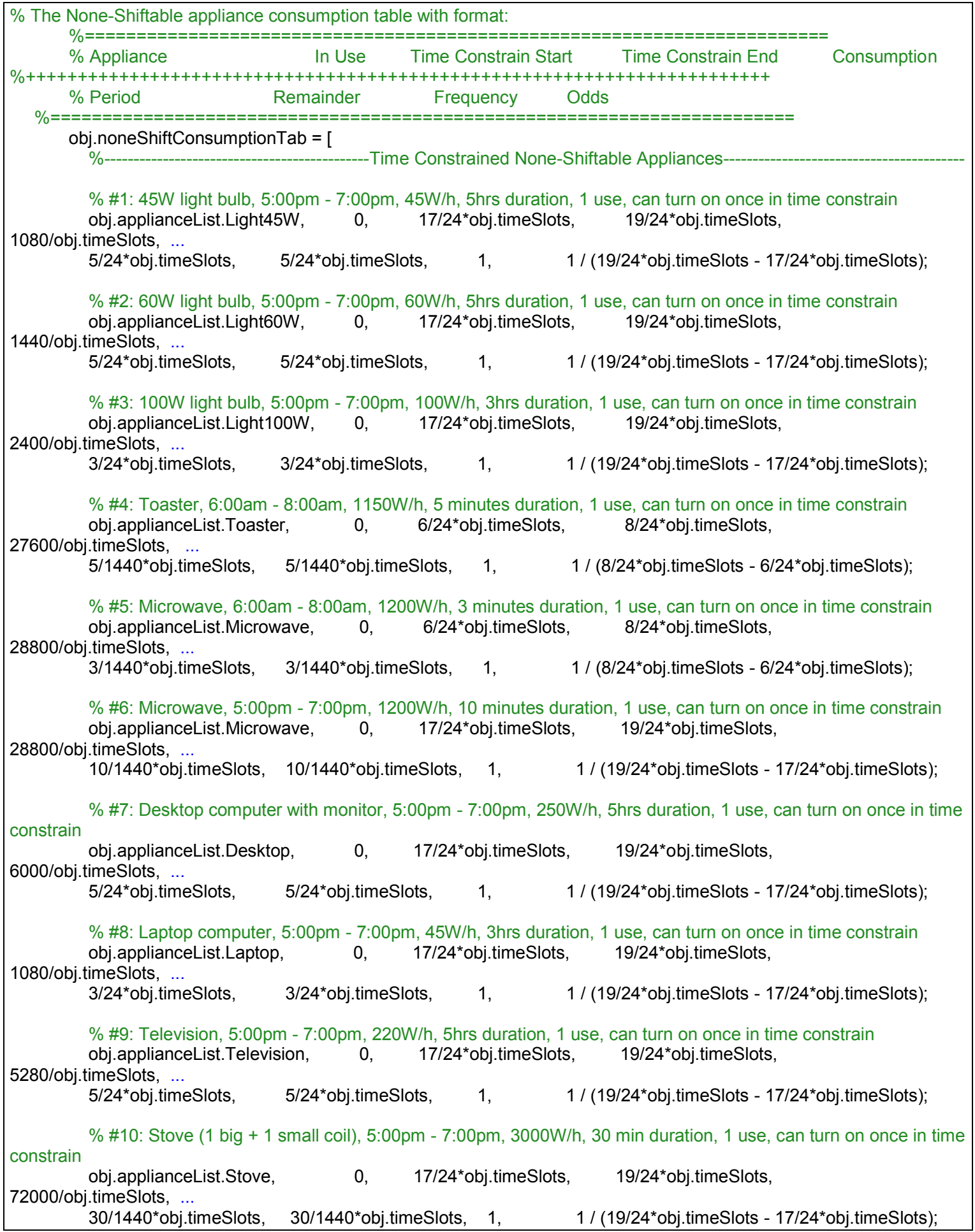


\% \#11: Hair dryer, 9:00pm - 11:00pm, 1200W/h, 10 min duration, 1 use, can turn on once in time constrain obj.applianceList.HairDryer, $\quad 0, \quad 21 / 24^{*}$ obj.timeSlots, $23 / 24^{*}$ obj.timeSlots,

28800/obj.timeSlots, ...

10/1440*obj.timeSlots, $\quad 10 / 1440^{*}$ obj.timeSlots, $1, \quad 1 /\left(23 / 24^{*}\right.$ obj.timeSlots $-21 / 24^{*}$ obj.timeSlots $)$;

\% \#12: Hair dryer, 6:30am - 8:00am, 1200W/h, 10 min duration, 1 use, can turn on once in time constrain obj.applianceList.HairDryer, $\quad 0, \quad 390 / 1440^{*}$ obj.timeSlots, $8 / 24^{*}$ obj.timeSlots,

28800/obj.timeSlots, ...

10/1440*obj.timeSlots, $10 / 1440^{*}$ obj.timeSlots, $1, \quad 1 /\left(8 / 24^{*}\right.$ obj.timeSlots $-390 / 1440^{*}$ obj.timeSlots);

\% \#13: 45W light bulb, 6:30am - 8:00am, 45W/h, 1 hour duration, 1 use, can turn on once in time constrain obj.applianceList.Light45W, $\quad 0, \quad 390 / 1440^{*}$ obj.timeSlots, $8 / 24^{*}$ obj.timeSlots,

1080/obj.timeSlots, ...

1/24*obj.timeSlots, $\quad 1 / 24^{*}$ obj.timeSlots, $\quad 1, \quad 1 /\left(8 / 24^{*}\right.$ obj.timeSlots $-390 / 1440^{*}$ obj.timeSlots);

\% \#14: 60W light bulb, 6:30am - 8:00am, 60W/h, 1 hour duration, 1 use, can turn on once in time constrain obj.applianceList.Light60W, $\quad 0, \quad 390 / 1440^{*}$ obj.timeSlots, $8 / 24^{*}$ obj.timeSlots,

1440/obj.timeSlots, ...

1/24*obj.timeSlots, $\quad 1 / 24^{*}$ obj.timeSlots, $1, \quad 1, \quad 1 /\left(8 / 24^{*}\right.$ obj.timeSlots $-390 / 1440^{*}$ obj.timeSlots);

constrain

\% \#15: Coffee maker, 6:30am - 8:00am, 1000W/h, 10 minutes duration, 1 use, can turn on once in time

obj.applianceList.CoffeeMaker, $\quad 0, \quad 390 / 1440^{*}$ obj.timeSlots, $\quad 8 / 24^{*}$ obj.timeSlots,

24000/obj.timeSlots, ...

10/1440*obj.timeSlots, $10 / 1440 *$ obj.timeSlots, $1, \quad 1$ / (8/24*obj.timeSlots - 390/1440*obj.timeSlots);

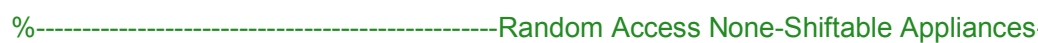

\% \#16: 45W light bulb, 0:00am - 12:00pm, 45W/h, 5 min duration, 10 use, can turn on 10 times in time

constrain

obj.applianceList.Light45W, $\quad 0, \quad$ 1/1440*obj.timeSlots, obj.timeSlots,

1080/obj.timeSlots, ...

5/1440*obj.timeSlots, $\quad 5 / 1440 *$ obj.timeSlots, $\quad 10, \quad 10$ / (obj.timeSlots $-1 / 1440 *$ obj.timeSlots);

$\%$ \#17: 60W light bulb, 0:00am - 12:00pm, 60W/h, 5 min duration, 10 use, can turn on 10 times in time constrain obj.applianceList.Light60W, $\quad 0, \quad 1 / 1440 *$ obj.timeSlots, obj.timeSlots,

1440/obj.timeSlots, ...

5/1440*obj.timeSlots, $\quad$ 5/1440*obj.timeSlots, $\quad 10, \quad 10$ / (obj.timeSlots - 1/1440*obj.timeSlots);

constrain

\% \#18: Microwave, 0:00am - 12:00pm, 1200W/h, 3 minutes duration, 3 use, can turn on 3 times in time

28800/obj.timeSlots.

3/1440*obj.timeSlots, $\quad 3 / 1440^{*}$ obj.timeSlots, $\quad 3, \quad 3 /$ (obj.timeSlots - 1/1440*obj.timeSlots);

constrain

\% \#19: Desktop computer, 0:00am - 12:00pm, 250W/h, 1 hrs duration, 3 use, can turn on 3 times in time

0, 1/1440*obj.timeSlots, obj.timeSlots,

6000/obj.timeSlots, ...

1/24*obj.timeSlots, $\quad 1 / 24^{*}$ obj.timeSlots, $\quad 3, \quad 3 /$ (obj.timeSlots $-1 / 1440^{*}$ obj.timeSlots);

\% \#20: Stove (1 big + 1 small coil), 0:00am - 12:00pm, 3000W/h, 15 min duration, 2 use, can turn on 2 times in time constrain

obj.applianceList.Stove, $\quad 0, \quad 1 / 1440^{*}$ obj.timeSlots, obj.timeSlots,

72000/obj.timeSlots, ...

15/1440*obj.timeSlots, $\quad$ 15/1440*obj.timeSlots, $2, \quad 2$ / (obj.timeSlots - 1/1440*obj.timeSlots);

constrain

\% \#21: Electric Oven, 0:00am - 12:00pm, 2000W/h, 45 min duration, 1 use in 3 days, can turn on once in time

obj.applianceList.Oven, $\quad 0, \quad 1 / 1440^{*}$ obj.timeSlots, obj.timeSlots,

48000/obj.timeSlots, ....

45/1440*obj.timeSlots, $\quad 45 / 1440 *$ obj.timeSlots, $\quad 1, \quad$ (1/3) / (obj.timeSlots - 1/1440*obj.timeSlots);

\% \#22: Hair dryer, 0:00pm - 12:00pm, 1200W/h, 5 min duration, 1 use, can turn on once in time constrain obj.applianceList.HairDryer, $\quad 0, \quad 1 / 1440^{*}$ obj.timeSlots, obj.timeSlots,

28800/obj.timeSlots, ... ];

5/1440*obj.timeSlots, $\quad 5 / 1440^{*}$ obj.timeSlots, $\quad 1, \quad 1 /$ (obj.timeSlots - $1 / 1440^{*}$ obj.timeSlots); 


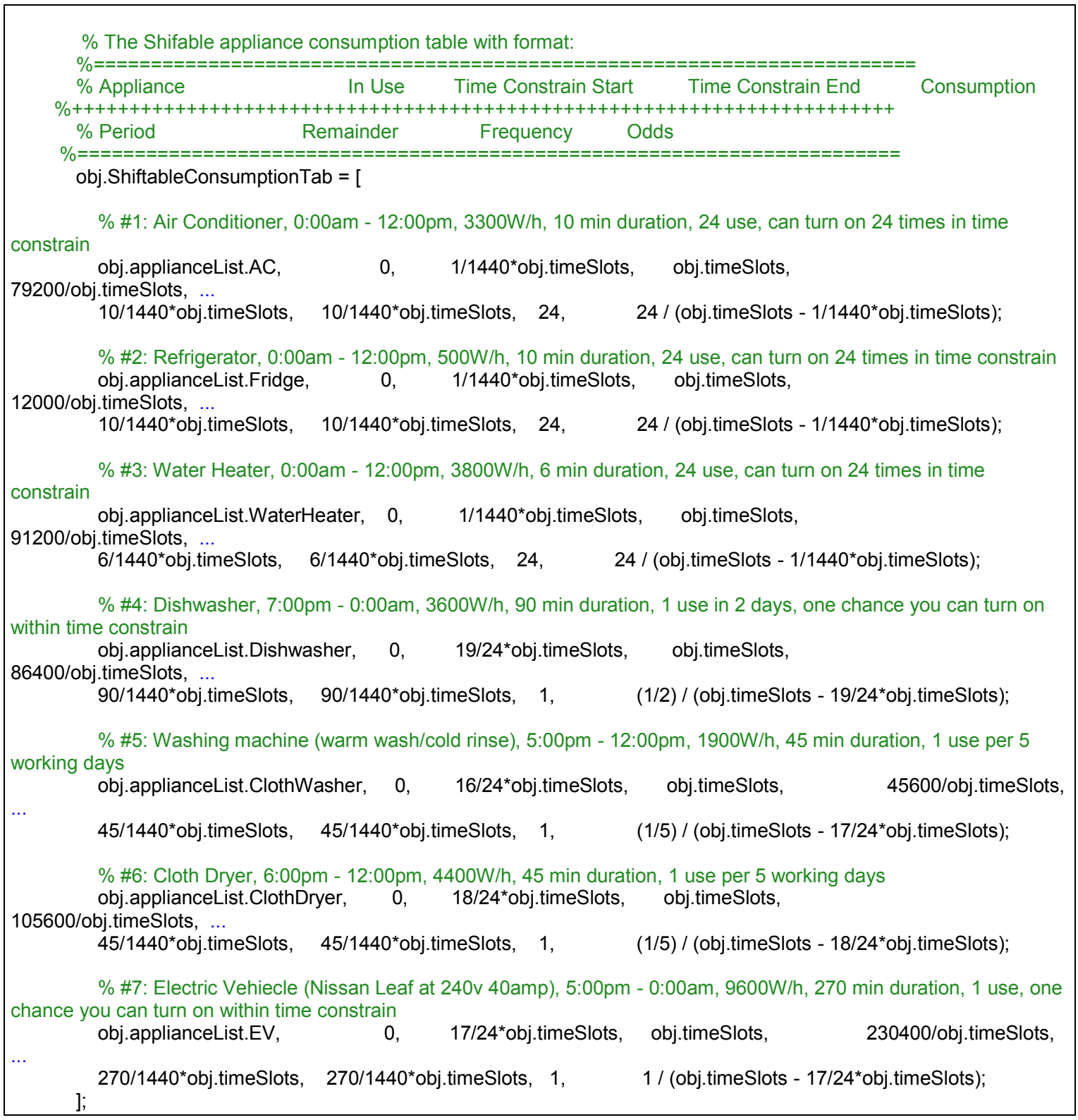




\section{References}

[1] “Obama Announces Winners of Smart-Grid Stimulus". October 27, 2009. Popular Mechanics. Last access on Nov. 2, 2012. http://www.popularmechanics.com/science/energy/efficiency/4335113

[2] “Canada Announces Funds for Smart grid Research". February 12, 2011. Smartmeters-telling it like it is. Last access on Nov. 2, 2012. http://www.smartmeters.com/the-news/smart-grid-news/1550-canada-announcesfunds-for-smart-grid-research.html

[3] "Plug Into the Smart grid". 2011. GE imagination at work. Last access on Nov. 2, 2012. http://ge.ecomagination.com/smartgrid/\#/landing_page

[4] "e-Radio-Inc and CBC/Radio-Canada team up to improve the way electricity is consumed". January 8, 2010. CBC Radio-Canda - News releases. Last access on Nov. 2, 2012. http://cbc.radio-canada.ca/en/media-centre/2010/01/january-8/

[5] "2011 State of the Consumer Report". Jan. 31, 2011. SmartGrid consumer collaborative. Last access on Nov. 2, 2012. http://smartgridcc.org/sgcc-2011-stateof-the-consumer-report

[6] "Revealing the Values of the New Energy Consumer". 2011. Accenture. Last access on Nov. 2, 2012.

http://www.smartgridnews.com/artman/publish/Business_Customer_Care/Revealin g-the-values-of-the-new-energy-consumer-3653.html

[7] "EcoPinion - Resurgence for Retail Electricity Choice and Competition Survey Report". April 2011. Ecoalign. Last access on Nov. 2, 2012.

http://www.smartgridnews.com/artman/publish/Business_Markets_Pricing/Resurge nce-for-retail-electricity-choice-and-competition-3654.html

[8] "Consumer Attitudes and the Benefits of Smart grid Technologies". Jan. 24, 2011. Parks Associates. Last access on Nov. 2, 2012. http://www.parksassociates.com/whitepapers/consumer-attitudes-and-the-benefitsof-smart-grid-technologies

[9] M. Chetty, D. Tran, R. E. Grinter. "Getting to Green: Understanding Resource Consumption in the Home". GVU Center and School of Interactive Computing Georgia Institute of Technology. Atlanta, GA, USA. ISBN: 978-1-60558-136-1. 
Dec. 8, 2008. Last access on Nov. 2, 2012.

http://delivery.acm.org/10.1145/1410000/1409668/p242-

chetty.pdf?ip $=134.117 .61 .232 \& C F I D=27906875 \&$ CFTOKEN $=63587948 \& \quad$ acm

$=1308711775 \mathrm{~d} 8578 \mathrm{a} 7 \mathrm{~d} 255 \mathrm{a} 6 \mathrm{e} 196 \mathrm{e} 58 \mathrm{e} 41 \mathrm{~cd} 305 \mathrm{a} 30 \mathrm{c}$

[10] "Smart Grid Insights: Smart Appliances". March 5, 2010. Zpryme. Last access on Nov. 2, 2012.

http://www.smartgridnews.com/artman/publish/End_Use_Appliances_Resources/S mart-Grid-Insights-Smart-Appliances-1979.html

[11] “Make Every Plug Talk”. 2010. Talkingplug-know your energy. Last access on June 10, 2011. http://talkingplug.com/

[12] "e-Radio-Inc and CBC/Radio-Canada team up to improve the way electricity is consumed". January 8, 2010. CBC Radio-Canda - News releases. Last access on June 10, 2011. http://www.cbc.radio-canada.ca/newsreleases/20100108.shtml

[13] "Energate announces commercial readiness of one-way FM Demand Response solution". October 19, 2010. E-Radio inc - technology you can use. Last access on June 10, 2011. http://e-radioinc.com/

[14] "ClimateTalk is a common information model developed for the exchange of information between disparate systems and devices". 2011. ClimateTalk Alliance. Last access on June 10, 2011. http://www.climatetalk.org/ClimateTalkTechnology/Overview/tabid/351/Default.as px

[15] "Communication Modularity - A Practical Approach to Enabling Residential Demand Response”. May 2011. EPRI-Electric Power Research Institute. Last access on June 10, 2011.

http://www.smartgridnews.com/artman/publish/Technologies_Communications/Co mmunication-modularity---a-practical-approach-to-enabling-residential-demandresponse-3737.html

[16] "EcoFactor ushers in the automated era of home energy management". June 9, 2011. Smartgridnews.com. Last access on June 10, 2011. http://www.smartgridnews.com/artman/publish/End_Use_Efficiency/EcoFactorushers-in-the-automated-era-of-home-energy-management-3740.html 
[17] “Cisco Smart grid Strategy: The Grid's First Operating System”. July 15, 2010. Smartgridnews.com. Last access on Nov 2, 2012. http://www.smartgridnews.com/artman/publish/Business_Strategy/Cisco-SmartGrid-Strategy-The-Grid-s-First-Operating-System-2698.html

[18] “Cisco Home Energy Management". 2010. Cisco public information - data sheet. Last access on June 10, 2011. http://www.cisco.com/web/consumer/pdf/data_sheet_c78_603194_v2.pdf

[19] "Google powermeter". 2011. Google.org.powerMeter. Last access on June 10, 2011. http://www.google.com/powermeter/about/

[20] "Delivery \& Smart grid". 2011. Microsoft Enterprise. Last access on June 10, 2011. http://www.microsoft.com/enterprise/industry/power-utilities/solutions/delivery$\underline{\text { smart-grid.aspx }}$

[21] "Who is dumber about home energy, Microsoft or Google?". July 5, 2011. Smartgridnews.com. Last access on Nov 2, 2012. http://www.smartgridnews.com/artman/publish/Technologies_Home_Area_Networ ks/Who-is-dumber-about-home-energy-Microsoft-or-Google-3792.html

[22] "The Smart Grid: A Pragmatic Approach". Electric Power System Reliability Assessment, 2009 Composite Version. Canadian Electricity Association. http://www.electricity.ca/media/SmartGrid/SmartGridpaperEN.pdf

[23] "Smart grid Resource Center". 2010. EPRI - Electric Power Research Institute. Last access on June 10, 2011. http://smartgrid.epri.com/

[24] "Statement of Policy on Modernization of Electricity Grid". May 14, 2009. SmartGridNews.com. Last Access on June 10, 2011. http://www.smartgridnews.com/artman/publish/Projects_Stimulus/Statement_of_P olicy_on_Modernization_of_Electricity_Grid-790.html

[25] “Modernizing Ontario's Electricity System: Next Steps”. May 2011. Independent Electricity System Operator. Last access on June 10, 2011. http://www.ieso.ca/smartgridreport

[26] M. Grabowski, G. Dziwoki. "The IEEE Wireless Standards as an Infrastructure of Smart home Network". Silesian University of Technology, Institute of Electronics. CCIS 39. Springer-verlag Berlin Heidelberg 2009. 
[27] CENELEC Representatives, "Interoperability framework requirements specification for service to the home (IFRS)", CENELEC Workshop Agreement, June 2010. Pages: 1-8

[28] P. Thinagaran et al., "Interoperability among Heterogeneous Systems in Smart home Environment", IEEE International Conference on Signal Image Technology and Internet Based Systems, 2008. Pages $177-186$.

[29] W. Kenneth, "Home Systems Standards: Achievements and Challenges", IEEE Communications Magazine, April 2002. Pages 152-159

[30] Ning Lu; Nguyen, T., "Grid Friendly ${ }^{\mathrm{TM}}$ Appliances - Load-side Solution for Congestion Management," Transmission and Distribution Conference and Exhibition, 2005/2006 IEEE PES, vol., no., pp.1269,1273, 21-24 May 2006

[31] J. Lu, T.I. Sookoor, V. Srinivasan, G. Gao, B. Holben, J.A. Stankovic, E. Field, and K. Whitehouse. "The smart thermostat: using occupancy sensors to save energy in homes". in Proc. SenSys. 2010. pp.211-224.

[32] Diane J. Cook, Michael Youngblood, Edwin O. Heierman, III, Karthik Gopalratnam, Sira Rao, Andrey Litvin, and Farhan Khawajia, "MavHome An Agent-Based Smart Home". Proceedings of the First IEEE International Conference on Pervasive Computing and Communications, 2003.

[33] Dae-Man Han, Jae-Hyun Lim, "Design and Implementation of Smart Home Energy Management Systems based on ZigBee", IEEE Transactions on Consumer Electronics, Vol. 56. No. 3, August 2010.

[34] Wang Chun-dong; Liu Xiao-qin; Wang Huai-bin, "A Framework of Intelligent Agent Based Middleware for Context Aware Computing," Natural Computation, 2009. ICNC '09. Fifth International Conference on, vol.6, no., pp.107,110, 14-16 Aug. 2009. doi: 10.1109/ICNC.2009.388

[35] Amir-Hamed Mohsenian-Rad, Alberto Leon-Garcia, "Optimal Residential Load Control with Price Prediciton in Real-Time Electricity Pricing Environments", IEEE Transactions on Smart Grid, Vol, 1, No. 2, September 2010.

[36] Ozturk, Y.; Senthilkumar, D.; Kumar, S.; Lee, G., "An Intelligent Home Energy Management System to Improve Demand Response," Smart Grid, IEEE Transactions on , vol.PP, no.99, pp.1,8, 0doi: 10.1109/TSG.2012.2235088 
[37] Samadi, P.; Mohsenian-Rad, H.; Wong, V.W.S.; Schober, R., "Tackling the Load Uncertainty Challenges for Energy Consumption Scheduling in Smart Grid," Smart Grid, IEEE Transactions on, vol.PP, no.99, pp.1,10, 0

[38] Palma-Behnke, R.; Benavides, C.; Lanas, F.; Severino, B.; Reyes, L.; Llanos, J.; Saez, D., "A Microgrid Energy Management System Based on the Rolling Horizon Strategy," Smart Grid, IEEE Transactions on , vol.PP, no.99, pp.1,11, 0

[39] Kim, S.-J.; Giannakis, G.B., "Scalable and Robust Demand Response With MixedInteger Constraints," Smart Grid, IEEE Transactions on , vol.PP, no.99, pp.1,11, 0 doi: 10.1109/TSG.2013.2257893

[40] Zhang, Y.; Lu, N., "Parameter Selection for a Centralized Thermostatically Controlled Appliances Load Controller Used for Intra-Hour Load Balancing," Smart Grid, IEEE Transactions on, vol.PP, no.99, pp.1,9, 0

[41] Z. Zhu, J. Tang, et al., "An Integer Programming and Game Theory Based Optimization for Demand-side Management in Smart Grid”, IEEE Globecom Workshops on Communication, Networking \& Broadcasting, 2011.

[42] Michael Swearingen, "Real Time Evaluation and Operation of the Smart Grid Using Game Theory", IEEE Rural Electric Power Conference on Communication, Networking \& Broadcasting, 2011.

[43] Hung Khanh Nguyen, Ju Bin Song, Zhu Han, "Demand Side Management to Reduce Peak-to-Average Ratio using Game Theory in Smart Grid", IEEE INFOCOM Workshop on Communications and Control for Sustainable Energy Ssytems: Green Networking and Smart Grids, 2012.

[44] Zheng, J.; Wang, X.; Men, K.; Zhu, C.; Zhu, S., "Aggregation Model-Based Optimization for Electric Vehicle Charging Strategy," Smart Grid, IEEE Transactions on, vol.PP, no.99, pp.1,9, 0

[45] Melike Erol-Kantarci, Janhangir H. Sarker, Hussein T. Mouftah, "Analysis of Plugin Hybrid Electrical Vehicle Admission Control in the Smart Grid", IEEE International Workshop on Computer Aided Modeling and Design of Communication Links and Networks, 2011. 
[46] Vandael, S.; Claessens, B.; Hommelberg, M.; Holvoet, T.; Deconinck, G., "A Scalable Three-Step Approach for Demand Side Management of Plug-in Hybrid Vehicles," Smart Grid, IEEE Transactions on , vol.PP, no.99, pp.1,9, 0

[47] Ota, Y.; Taniguchi, H.; Nakajima, T.; Liyanage, K.M.; Baba, J.; Yokoyama, A., "Autonomous distributed $\mathrm{V} 2 \mathrm{G}$ (vehicle-to-grid) considering charging request and battery condition," Innovative Smart Grid Technologies Conference Europe (ISGT Europe), 2010 IEEE PES , vol., no., pp.1,6, 11-13 Oct. 2010

[48] Gouveia, C.; Moreira, J.; Moreira, C.L.; Pecas Lopes, J.A., "Coordinating Storage and Demand Response for Microgrid Emergency Operation," Smart Grid, IEEE Transactions on, vol.PP, no.99, pp.1,11, 0

[49] Steffen Schutte, Stefan S, et al., "Mosaik: A Framework for Modular Simulation of Active Components in Smart Grids", IEEE International Workshop on Smart Grid Modeling and Simulation, 2011.

[50] Jin Young Choi, Jae-Hoon Kim, "An Integrated Simulaiton Framework of a Virtual Test-bed for Smart Grid", IEEE International Conference on Computers and Industrial Engineering, 2010.

[51] Andren, F.; Stifter, M.; Strasser, T.; Burnier de Castro, D., "Framework for coordinated simulation of power networks and components in Smart Grids using common communication protocols," IECON 2011 - 37th Annual Conference on IEEE Industrial Electronics Society, vol., no., pp.2700,2705, 7-10 Nov. 2011

[52] Godfrey, T.; Mullen, S.; Dugan, R.C.; Rodine, C.; Griffith, D.W.; Golmie, N., "Modeling Smart Grid Applications with Co-Simulation," Smart Grid Communications (SmartGridComm), 2010 First IEEE International Conference on , vol., no., pp.291,296, 4-6 Oct. 2010

[53] Hua Lin; Veda, S.S.; Shukla, S.S.; Mili, L.; Thorp, J., "GECO: Global EventDriven Co-Simulation Framework for Interconnected Power System and Communication Network," Smart Grid, IEEE Transactions on, vol.3, no.3, pp.1444,1456, Sept. 2012

[54] "Energy Consumption of Major Household Appliances Shipped in Canada, Trends for 1990-2009". 2009. Natural Resources Canada. Cat. No. M141-16/1-2009(Print). ISSN 1927-5846. 
[55] “Major Appliances". Natural Resources Canada. Date Modified:2011-10-26. Last access on Nov. 15, 2012. http://oee.nrcan.gc.ca/equipment/appliances/12377

[56] "ENERGY STAR Publications". 2012. ENERgY STAR. Last access on Nov 15. 2012.

https://www.energystar.gov/index.cfm?fuseaction=publications.showPublications

[57] "Saving Electricity". 1998-2012. Michael Bluejay, Inc. Last Access on Nov. 15, 2012. http://michaelbluejay.com/electricity/

[58] "PROGRAMS \& INCENTIVES - PLUSTM". 2005-2012. HydroOttawa. Last Access On Nov. 15, 2012. http://www.hydroottawa.com/residential/saveonenergy/programs-andincentives/peaksaver/

[59] "Day-Ahead Prices in Dollars/KWH”. 2012-11-17. Ameren. Last Access on Nov. 17, 2012. https://www2.ameren.com/RetailEnergy/realtimeprices.aspx

[60] "Residential Customers - Electricity Charge". 2005 - 2012. HydroOttawa. Last Access on Nov. 17, 2012.

http://www.hydroottawa.com/residential/index.cfm?lang=e\&template $\mathrm{id}=156$

[61] "Company Overview". 1984-2012. The MathWorks, inc. Last Access on Dec. 12, 2012. http://www.mathworks.com/company/aboutus/?s_cid=wiki_mathworks_1

[62] “Application and Network Performance Management".2012. OPNET Technologies, Inc. Last Access on Dec. 12, 2012. http://www.opnet.com/

[63] Yigzaw G. Yohanis, Jayanta D. Mondol, Alan Wright, Brian Norton. "Real-life energy use in the UK: How occupancy and dwelling characteristics affect domestic electricity use". Energy and Buildings 40 (2008) 1053-1059. Northern Ireland, UK.

[64] "Save on energy". 2012. Ontario Power Autority. Last Access on Mar. 11, 2013. https://saveonenergy.ca/Consumer/Programs/PeaksaverPlus.aspx 